\title{
The influence of attention on motion processing
}

\author{
Dissertation \\ for the award of the degree \\ "Doctor rerum naturalium" \\ Division of Mathematics and Natural Sciences \\ of the Georg-August-Universität Göttingen \\ submitted by \\ Valeska Marija Stephan
}

from Siegen

Göttingen, 2012 
Doctoral thesis committee:

Prof. Dr. Stefan Treue (Advisor, First Referee)

Cognitive Neuroscience Laboratory

German Primate Center (DPZ)

Kellnerweg 4

37077 Göttingen

Prof. Dr. Alexander Gail (Second Referee)

Sensorimotor Group, BCCN

German Primate Center (DPZ)

Kellnerweg 4

37077 Göttingen

Prof. Dr. Julia Fischer

Cognitive Ethology Laboratory

German Primate Center (DPZ)

Kellnerweg 4

37077 Göttingen

Date of the oral examination: $\quad 25.10 .2012$ 
Herewith I declare that I have written this thesis independently and with no other aids and sources than quoted.

Göttingen, 31. August 2012

Valeska Marija Stephan 
"Die moderne Wissenschaft hat der Menschheit die Notwendigkeit des Wanders auferlegt. Ihr fortschreitendes Denken und ihre fortschreitende Technik machen den Übergang durch die Zeit, von Generation zu Generation, zu einer Wanderschaft in unbekannte abenteuerliche Gewässer. Der Segen des Wanderns liegt gerade darin, dass es gefährlich ist und Fertigkeiten verlangt, um Übel abzuwehren. Daher müssen wir erwarten, dass die Zukunft Gefahren enthüllen wird. Es ist die Aufgabe der Zukunft, gefährlich zu sein; und es gehört zu den Verdiensten der Wissenschaft, dass sie die Zukunft für ihre Aufgaben ausrüstet."

Alfred North Whitehead, Science and the modern world (1925) 


\section{Acknowledgements}

First of all, I want to thank Stefan Treue for gving me the fantastic opportunity to work in his laboratory. I'm extremly grateful for being able to study under his guidance and to benefit from his mentorship, support and experience throught my time here. Furthermore, I thank Alexander Gail and Julia Fischer as member of my thesis committee, for their constant support and advice throughout my time as a graduate student.

I thank Leonore Burchardt, Dirk Prüsse, Sina Plümer and Klaus Heisig for invaluable help with taking care of the monkeys and always helping out whenever help was necessary. Thanks to Beatrix Glaser for her reliable administrative and tax support.Thanks to Ralf Brockhausen and Kevin Windolph for quick and reliable IT-support. Thanks to the great people at the GGNB office, for their support and helping to calm nerves.

A special thanks to Katharina Anton-Erxleben for being a great teacher and preparing me for the good as well as bad times lying ahead. Thanks to Anja Lochte for her hard work in preparing the project and being such a pleasant colleague to work side by side with. Thanks to Rui-Feng Liu for major help in getting started with programming and the help with the monkeys. Thanks to Vladislav Kozyrev for being a big help in programming and data analysis.

Thanks to my awesome office mates Cliodhna Quigley, Antonio Calapai and Sonia Baloni for being simply awesome. A very special thanks to my PhD-buddy of the past five years Sonia Baloni, whos company was -especially in the last weeksinvaluable. Thanks to Shubo Chakrabarti, Philipp Schwedhelm, Vera Marks, Pablo Martinez, Pierre Morel, Stephanie Westendorff, Sebastian Lehmann, Stefan Schaffelhofer, Katharina Menz, Thiemo Daldrup and Stefanie Schuldes for making work days much more enjoyable and for sharing great free time activities. Thanks to the crew of MJN Air for never failing to cheer me up. Yellow car! I also thank the other members and former members of the cognitive neuroscience lab for discussions, help, fun and an all together an extremely pleasant atmosphere in the lab throughout all the years. 
viii

The biggest thanks goes out to my family \& Wolfgang Müller for their constant support, help and all the rest, which would take up entirely too much space to be listed here. Thank you! 


\section{Contents}

1 Introduction .....................................................................................

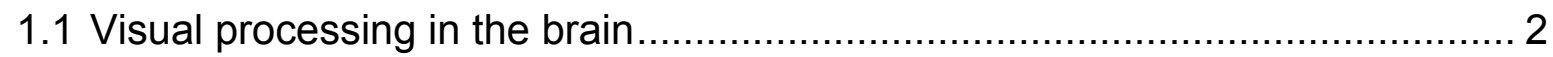

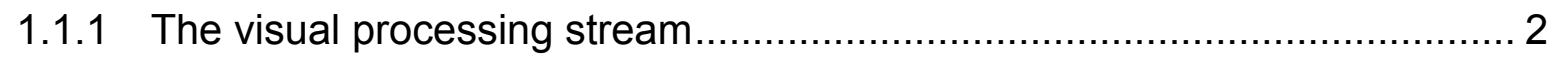

1.1.2 Motion processing in the visual stream .......................................... 3

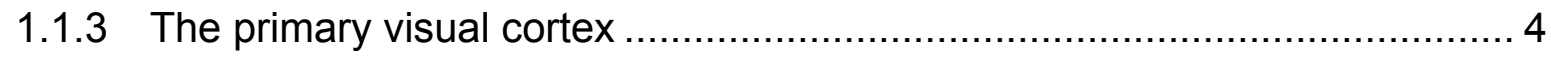

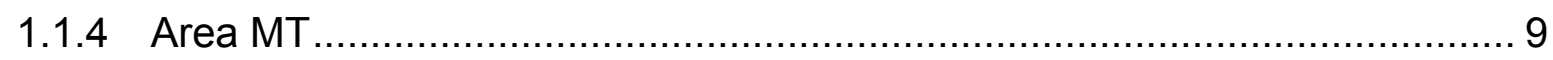

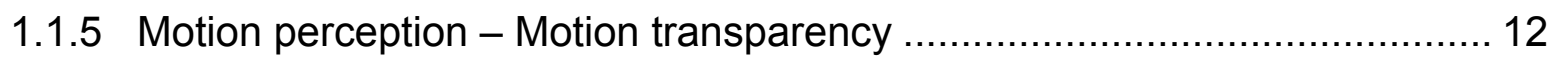

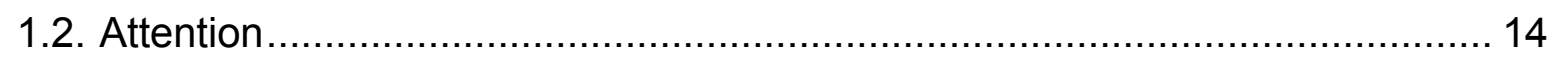

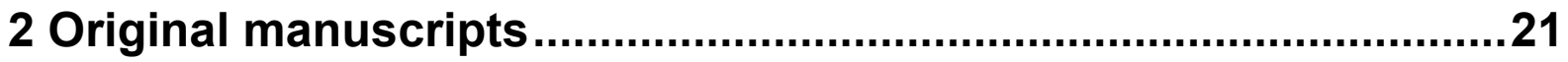

2.1 Attentional modulation of neuronal response in macaque area MT to

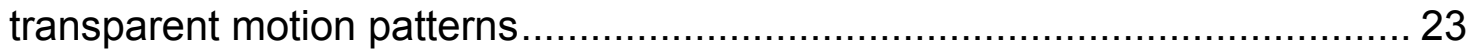

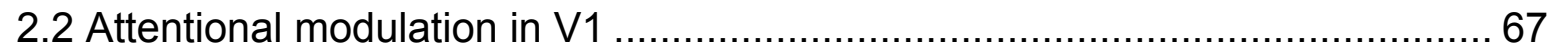

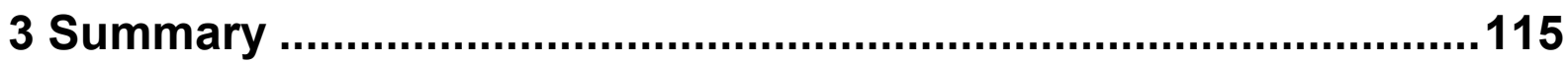

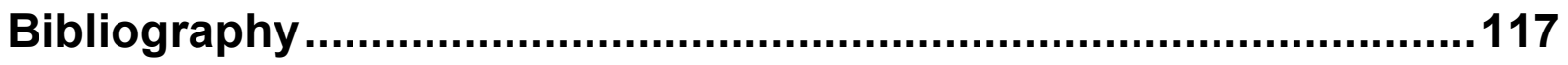

Curriculum Vitae .........................................................................128 



\section{Introduction}

Seeing is a multi-staged process in the brain. Visual information enters the visual system through the retina and is then projected via the thalamus to the primary visual cortex (V1). Here the visual input is segregated into two major pathways, which are defined according to the features of the visual input they mainly process. Motion is an important defining feature of the dorsal pathways and is processed by areas including area MT.

Area MT has been identified as being involved in motion processing since its characterisation in the mid 70's. A large body of work has since identified its anatomical and functional features and the cognitive mechanism, which influence its processing of visual sensory information.

As we will see in details below, attention is known to have a large impact on the sensory responses in area MT. However, research has predominately focused on simple motion stimuli involving presentations of one motion direction at a time. We were interested how attention might influence motion processing of more complex stimuli. Therefore, we presented transparent motion stimuli to a monkey and observed MT cells' reactions under different attentional states. Furthermore, we were interested in the question of the attentional mechanisms, acting on motion processing. In particular we were interested on the origin of the well-known effects of spatial attention on sensory processing in MT. To address this question, we recorded responses in the primary visual cortex $\mathrm{V} 1$ with an experimental paradigm which has been shown to produce strong effects of spatial attention in area MT.

In this introduction we will introduce the visual system and in particular the processing of visual motion. We will focus on the two areas which are of particular interest for our research questions, namely V1 and area MT. Furthermore, we will review concepts of the processing of transparent motion as compared to single motion stimuli. Finally, in the introductory paragraph on attention, we will give a broad overview on the topic, involving effects and theoretical work and highlight findings, that are of particular importance for our studies. 


\subsection{Visual processing in the brain}

Visual information is processed in a cascadic fashion. The fundamental principle can be broadly described as going from extracting basic features of the visual content in the lower parts of the hierarchy, to very specific parts in the higher areas in the hierarchy (Maunsell \& Cook, 2002; Van Essen \& Maunsell, 1983). Although the basica ideas involved in visual processing have been characterized, many features of the visual processing stream are still left to be explored. To date, more then 30 areas have been identified to be involved in the processing of visual information, yet even the most extensively researched ones have not been fully described in their functionality (Van Essen \& Gallant, 1994). An extensive network of interconnections between and within areas make the task of decoding the visual processing stream in its details particularly challenging (Felleman \& Van Essen, 1991).

In the following paragraphs we will sketch out the broader outlines of the visual processing stream, with a special focus on motion processing, which predominately involve the primary visual cortex (V1) and the medial temporal cortex (area MT). Both of these areas and their characteristics and interconnections are of particular relevance for the studies included in this thesis, therefore we will focus on these aspects.

\subsubsection{The visual processing stream}

Visual information comprising our environment enters the visual processing stream via the retina.

Light, in the form of electromagnetic waves in the visible spectrum, falls onto the retina and hyperpolarizes the intracellular potentials of photoreceptors. In downstream retinal neurons the reaction of the photoreceptors is transformed into action potentials. Through a network of interneurons these signals are transferred to the retinal ganglion cells. Retinal ganglion cells respond to a spatially restricted area in the retina - the so called receptive field. Receptive fields are universal properties of visual neurons and change in certain characteristics, such as the size they subtend in the visual field, along the processing stream (Alsonso \& Chen, 
2008, for review). Ganglion cells project via the optic nerve and optic tract to the lateral geniculate nucleus (LGN). In the LGN the stream of visual information is segregated to the different parvo- and magnocellular layers, before it is further projected into V1 via the optic radiation. Within V1 the visual information is separated into two streams of processing, which show clear anatomical, as well as functional differences (Van Essen \& Maunsell, 1983; Ungerleider \& Mishkin,1982). The ventral stream, also termed the 'what'-pathway gets its inputs mainly from the parvo-cellular layers. It projects from V1 into V2 to V4 in the temporal cortex. The key characteristics of the processing properties in this stream are processing of colour, form recognition, object representation and orientation (Van Essen \& Maunsell, 1983). Higher areas in the ventral stream include, among others, TEO and IT, which are sensitive to complex shapes and FFA, which show a high selectivity for faces (Tsao et al., 2006).

The second stream which V1 projects to is called the dorsal pathway ('where' pathway). It gets its input predominantly from the magnocellular layers of the LGN. Key characteristics of the dorsal pathway are the processing of spatial stimulus properties, representation of object locations, motion processing and the control of eye movements (Van Essen \& Maunsell, 1983; Van Essen \& Gallant, 1994). Information from V1 is projected to V2, V3 and to the areas MT and MST. Higher cortical areas are FST, VIP and LIP (Ungerleider \& Desimone,1986).

Note that despite the functional and anatomical separation of these two streams, they are not completely independent as extensive interconnections between areas is a common feature in usual corticlal processing. It is for example known that there is some amount of input from the parvocellular layers into the dorsal pathway (Sincich \& Horton, 2005; Nassi et al., 2006) and connections from the dorsal stream to areas which process colour and form (Ungerleider \& Desimone,1986).

\subsubsection{Motion processing in the visual stream}

As outlined above, the visual system is specialized in analysing different features of visual information in a cascade of processing steps, which are localized in different areas along the processing pathways. Central to this thesis is the processing of visual motion. 
Fast and accurate processing of visual motion is essential for the survival of an organism. Without the ability to judge motion correctly, perception of the position and speed of a predators or that of self-motion of an individual are affected and interactions with the environment are crucially impaired (Zihl et al., 1983).

Motion processing accurs in the dorsal pathway. Areas MT, MST and FST play key roles in the correct processing of motion (Ungerleider \& Mishkin, 1982). However, direction selective cells can already be found in the primary visual cortex. In 1959 Hubel and Wiesel (1959) studied the primary visual cortex in anaesthetised cats and found cells, which responded strongly to stimuli moving in a particular direction (termed 'preferred direction'), whereas these cells showed a diminished response to the opposite direction of motion (anti-preferred direction), and intermediate responses to directions in between. The responses of these direction-selective cells can be well described by a Gaussian shaped distribution as a function of stimulus direction, a so called tuning curve. A high number of these direction-selective cells in V1 can be found in the layer 4B, which in turn projects either via area V2 or directly from V1 to MT (Felleman \& Van Essen,1991; Livingstone, 1998).

In the following paragraphs we will review the fundamental anatomical and functional properties of the primary visual cortex, giving a basic outline of ist connectivity and highlighting its function in the visual processing hierarchy.

\subsubsection{The primary visual cortex}

The primary visual cortex (V1) is perhaps the most well studied brain area in the visual cortex. First described in 1855 it has been the object of research ever since. Several important findings, which we take as given nowadays, have been discovered in $\mathrm{V} 1$. The most noteworthy is perhaps the aforementioned discovery of visual receptive field structures by Hubel \& Wiesel in the early 60's (Hubel \& Wiesel, 1959; Hubel, 1963). But other important features of the visual system have also first been described in the primary visual cortex, including the computational principle of normalization, which was proposed in the early 90's to account for nonlinear properties in V1 (for review, see Carandini \& Heeger, 2012). 
The primary visual cortex occupies a large area in the occipital lobe of the mammalian brain. In the macaque the size is about $1200 \mathrm{~m}^{2}$ (Blasdel \& Campbell, 2001). It has a particularly high density of neurons, which can be taken as an indication of its importance as a part of the visual system.

V1 consists of six layers. The well defined, striped anatomical structure of the tissue can be seen without any magnification or staining, hence the name 'Striate Cortex'. Layer 4 can furthermore be separated into 4 sublayers (4A, 4B, 4Ca, $4 \mathrm{Cb})$. The magno- and parvocellular layers coming from the LGN are the prime input to $\mathrm{V} 1$.

Cells in the magnocellular layer show responses to visual stimuli of low spatial frequency and are sensitive to even low changes in luminance. This makes them well suited for detecting luminance changes and moving stimuli (Callaway, 1998), whereas cells in the parvocellular layer show an enhanced response towards colour stimuli (Gegenfurtner \& Kiper, 2003). The magno- and parvocellular projections enter the cortex through layer 4, where the input from the magnocellular layers is primarily projected into sublayer $4 \mathrm{Ca}$ and parvocellular layers project mainly to $4 \mathrm{Cb}$. This subdivision ensures that the functional separation of these two streams is maintained after leaving the LGN. Separation and a strict organization of the visual information is obtained in the primary visual cortex through a sophisticated anatomical arrangement of the input information (Callaway, 1998). We find distinct patterns of cell formations. Prominent features of these anatomical arrangements are for example the orientation columns in layer 4 , which hold orientation selective cells in a sophisticated, well defined functionalanatomical structure, or cytochrome oxidase-blobs in layer 3 , which appear to be partly responsible for the organization of colour selective cells (Livingstone \& Hubel, 1984; Gegenfurtner, 2003). Orientation columns, blobs and in addition ocular dominance columns are organized in socalled hypercolumns. One hypercolumn refers to a set of orientation-, ocular dominance columns and blobs. The organization of hypercolumns is very precise and ordered, each spanning around a 1x1mm in the cortex (Hubel \& Wiesel, 1974a,b). Whereas columns and hypercolumns are thought to represent a vertical connectivity among groups of cells, the horizontal connectivity is ensured through axon collaterals of pyramid cells. The main aim of the vertical connections is the connectivity among cells, 
which have the same feature selectivity but diverge in the spatial position of their receptive fields. Please note that while basic anatomical structures of the primary cortex are not a focus of argument anymore, the connectivity of the primary visual cortex (within-area, as well as between areas) is still a hotly debated topic. Reviewing the current debate in details, beyond the scope of this introduction.

In addition to the highly sophisticated and impressively structured within-area connectivity, V1 possesses a similarly impressively structured network of connections to a vast number of cortical as well as subcortical areas (Felleman \& Van Essen, 1991; Gattass et al., 2005; see Callaway, 1998 \& Schmolesky, 2007 for review). We can find extensive networks of direct feedforward projections from and to V1 from areas such as the pulvinar, LGN, V2, MT and FEF. On the other hand we find extensive feedback projections from and to $V 1$ from areas e.g. V2, V4, LIP, MT or the pons Projections to subcortical layers depart mainly from layer 6 and 5 , whereas outputs to cortical layers are prefrably found in layer $2 / 3$ (Carandini, 2012).

Another aspect, reflecting the astonishing systematics and order in which V1 processes visual information is its retinotopy. The visual information entering area V1 represents the complete visual field and is retinotopically organized (e.g. Blasdel \& Campbell, 2001), which means nearby points in the visual field are represented by nearby points in the cortex. The local representation of the visual field is very precise, whereas the global representation is not. The uneven representation of the visual field can already be seen in the retina. The clustering of light receptors in the retina is much more dense around the fovea, which leads to an overrepresentation of the foveal areas as compared to the more eccentric areas of the visual field. However, this specific representation of the distorted global representation of the visual field cannot fully be attributed to receptor clustering in the retina. Adams \& Horton (2003) found in the squirrel monkey that the central 8 degree of the retina contained $28 \%$ of the retinal ganglion cells, but nevertheless correspond to $48 \%$ of the cortex (Fig. 1). While the mechanism behind this particular phenomenon is not fully understood, the overrepresentation of the central areas around the fovea is obviously useful, as organisms do foveate towards objects of interest and vision has to be specifically accurate in this area. This overrepresentation is extremely pronounced in the primary visual cortex and is termed visual distortion (Daniel \& Whitteridge, 1961; Blasdel \& Campbell,2001; 
Fig. 2). Cells with receptive fields falling on the visual space close to the fovea take up a large part of the most lateral parts of the cortex. Moving more medial on the cortex, receptive fields get bigger in size (up to 4/8 degrees) and cover also more eccentric areas in the visual space (Hubel \& Wiesel, 1974b). In humans roughly $50 \%$ of the primary visual cortex covers $2 \%$ of visual space (Wandell \& Smirnakis, 2009; Carandini, 2012).

Figure 1 - Representation of the contralateral visual hemifield in area V1 of the macaque. The diagram shows representation of vertical, horizontal, and oblique meridia on the surface of the cortex up to an eccentricity of 8 degrees. The vertical meridian (indicated by black half arrows) runs along the outer boundary of V1. Because of an exponential change in the magnification with eccentricity, it bulges out, encircling most of $\mathrm{V} 1$. The horizontal meridian is represented by a crossed pattern.

It is important to note that the surface representation of space is inverted. Upper parts are represented in the lower (posterior) and lower parts of visual space are represented in the upper (anterior) parts of V1. (from Blasdel (2001), with permission)

Cells in area V1 respond predominantly to rather basic components of a visual stimulus, like spatial or temporal frequency (Movshon et al., 1978). But unlike other areas in the visual cortex, we do find a wide variety of stimulus features to which cells in area V1 respond, ranging from colour (Gegenfurtner \& Kiper, 2003) to disparity (Cumming \& DeAngelis, 2001). Covering all details of the tuning properties for each feature is however, not be the purpose of this introduction, so we will focus on the orientation and direction tuning of $\mathrm{V} 1$ cells. 
Orientation tuning is one of the key characteristics of V1 cells, whereas direction tuning is only found in approximately $30 \%$ of the population (Snowden et al., 1991). Orientation tuning can be clearly attributed to processes within the area itself, because unlike for example responsiveness towards colour, it is not present in the projections from the LGN (Carandini, 2012). Orientation selectivity for simple cells can be directly traced to the shape of the receptive fields (Adelson \& Bergen, 1985, Carandini et al., 1996). Receptive fields of simple cells consist of an On- and Off-region. Presenting a stimulus within the On-region elicits a response from the neuron, while presenting a stimulus within the Off-region does not. On- and Offregions of simple cells are elongated so that their preferred stimulus is an oriented bar. The structure of complex cells receptive fields is more sophisticated and their orientation selectivity cannot be derived from the shape of the receptive field. However, it can be traced back to the simple cells which provide input to the complex cell. All input cells have the same orientation selectivity, hence passing their selectivity on to the complex cell. Direction selectivity can be explained likewise by extending the spatial representation of the receptive field with a dimension depicting time (Adelson \& Bergen, 1985). Imagine a vertically oriented light bar moving to the right. Tracking the movement of the bar over time would result in a tilted line in a space-time plot, in which steepness depends on velocity.

About $30 \%$ of cells in the primary visual cortex show direction selectivity, most of which are located in layer 4 (Snowden et al., 1992). Additionally, a small layer of direction selective cells can also be found in layer 6 (Livingstone, 1998, Gattass et al. 2005). Although cells in area V1 show direction selectivity, it must be noted, that in general V1 neurons are clearly not as specialized for the processing of motion as area MT in the extra-striate cortex. Snowdon and colleagues compared responses to moving random dot patterns in area $\mathrm{V} 1$ to responses in area MT. While they could show that direction selective cells in V1 did show a clear tuning to a preferred direction, the average tuning selectivity was not near as pronounced as in cells of area MT. They computed the directional index for cells from both areas and while MT cells showed a strong directionality (mean = 1.01), the population of $\mathrm{V} 1$ cells showed a far less specific directionality (mean $=0.44$ ). However, Movshon and Newsome (1996) studied cells in V1 and MT, which were directly connected. They could show that cells in V1 which project directly to MT 
cells show a high direction selectivity, which is around in the magnitude of MT cells. So while V1 cells as a population are not specifically direction selective, those single cells which project directly to MT cells do show strong direction selectivity. We will further elaborate on direction selectivity and the differences between V1 and MT at a later point in this introduction.

In the last paragraphs we have briefly reviewed the main anatomical and functional structures of the primary visual cortex; including retinotopy and connectivity. Furthermore we looked at two of the stimulus features V1 cells respond to and outlined their origin in the receptive field structure of the cells. Concluding, it can be stated that while $\mathrm{V} 1$ is one of the prime targets for vision research and a lot of important findings have been made studying this particular area, it can also safely be said that it is also a prime example for the principle of scientific work that with every answer, ten new questions arise.

Moving along the dorsal stream of visual processing, one of the major direct projections from the primary visual is the higher visual area MT. In the following paragraph we will review the fundamental characteristics of this area and highlight its function in the processing of visual motion.

\subsubsection{Area MT}

Area MT was firstly described as a motion selective area in a range of studies by Zeki and colleagues in the 70's (Born \& Bradley, 2005). Further work (e.g. Newsome et al., 1989; Salzman et al., 1990) established the area as being responsible for the perception of motion in the visual cortex. Salzman et al. (1990) trained monkeys to identify a direction of motion in a random dot pattern with different degrees of motion coherence. While the monkey did the task they electrically stimulated cells in area MT and could show a leftward shift of the psychometric function of the monkey, indicating that with stimulation less coherence of the stimulus was needed to perceive the direction. This result showed a causal connection between motion perception and area MT.

Area MT is positioned in the lower bank of the superior temporal sulcus (Gattass \& Gross, 1981). It is well defined by its high density of myelination (Gattass \& Gross, 
1981). MT is also known as V5 or hMT (MT+) in the human cortex (Born \& Bradley (2005)). Within the dorsal pathway, MT is embedded between the lower visual area V1 and higher areas such as MST and FST, giving input into the analysis of optic flow. Furthermore it shows connectivity to areas LIP and FEF, which are involved in the generalization of eye movements. Connections to areas in the ventral pathway are also known (Van Essen \& Maunsell (1983)). Although area MT receives inputs from a variety of areas, the most prominent one is direct input from area V1, specifically from the magnocellular layer in sublayer 4B. Like area V1, area MT is also retinotopically organized (Gattass \& Gross, 1981). The visual field is completely represented, with an overrepresentation of the region around the fovea (Van Essen et al., 1981). While the size of the receptive fields in V1 is strongly depended on eccentricity with the size substantially increasing towards the periphery, the influence of eccentricity on the receptive field size is less pronounced in area MT (Gattass \& Gross, 1981). The average size of a MT receptive field is 10 degrees (e.g. Gattass \& Gross, 1981, Born \& Bradley, 2005). Whereas in V1 only a small number of cells show a defined direction-selectivity, the number of direction-selective cells in MT is very high. Around $95 \%$ of the cells in MT are direction selective (Albright et al., 1984). As mentioned earlier, MT cells furthermore show very strong direction selectivity (Snowden et al., 1992). Other properties of MT neurons are speed selectivity (Orban et al., 1986) and sensitivity to motion disparity (Prince et al., 2000). The direction-selective cells in MT are organized in columns (Albright et al., 1984), which overlap with columns of disparity-tuned cells (DeAngelis \& Newsome, 1999). Direction columns of smoothly varying preferred directions are thought to run along columns, which have locally opposite preferred directions. Neurons with similar preferred speeds are also arranged in clusters, but strict columnar organization has not been found (Liu \& Newsome, 2003).

The size of MT receptive fields compared to V1 receptive fields suggest that MTs prime task is the summation of short-range motion information projected from area V1 over a larger spatial extent. It could be shown however that the spatial scale over which MT processes motion is similar to V1 (Churchland et al., 2005). Furthermore, characteristics of V1 inputs are detectable in MT receptive fields (Churchland et al., 2005, Livingstone, 1998). It also seems that MT cells do not contribute to the generation of motion selectivity, as $\mathrm{V} 1$ cells projecting directly to 
MT already show a direction selectivity similar in magnitude to MT cells (Movshon et al., 1996). Work from Movshon et al. (1985) in the mid 80's however could show that MT cells have a large impact on solving the aperture problem. V1 neurons, due to their limits in receptive field size and orientation, are faced with the problem of detecting the movement of moving edges within their receptive fields correctly. V1 cells are only capable of encoding motion components orthogonal to their preferred orientation and can therefore be considered to extract local motion information. MT cells on the other hand can overcome this problem because of their larger size in receptive fields. To correctly detect global motion signals, a pooling over the outputs of local motion units tuned to different directions of motion is necessary. It could be shown that MT cells are well capable of this task. Furthermore, MT neurons employ mechanisms to reduce noise in the motion signal; for example via motion opponency (suppressive effect of non-preferred direction to responses towards preferred direction, when presented simultaneously (Snowden et al., 1990 , details will be discussed in a later chapter) or gain normalization (scaling of neuronal responses to the total amount of neural activity (Simoncelli \& Heeger, 1998; Heuer \& Brittem, 2002). MT cells are also considered to contribute to motion-related segmentation of the visual image due to the centersurround structure of the receptive fields (Bradley \& Andersen, 1998). This antagonistic structure favours segmentation of a moving stimulus from its background.

These examples show the strong impact of MT cells on the perception of motion. Due to their specific characteristics of large receptive fields, center-surround interactions and their feature preferences -combining disparity, speed and motionMT cells are able to put motion signals they receive from the tight connection to V1 into a perspective and contribute vastly to the decoding of global motion in the visual scene.

In the following paragraph we will extend our review on the processing of motion in area MT by discussing a special case of motion stimuli and the role of MT in processing those stimuli. 


\subsubsection{Motion perception - Motion transparency}

A special case of a visual motion stimulus is transparent motion. Transparent motion refers to the case when two (or more) different directions of motion occupy the same visual space. While in the laboratory environment visual stimuli are usually reduced to their simplest components and often only contain one specific direction of motion, in the natural environment this is hardly ever the case. Natural scenes consist of a high number of visual features, like motion, which are often overlapping. For example moving leaves in the wind or raindrops on the windows of a moving car. The visual system is confronted with the demanding task of detecting the different objects and surfaces and putting them in the right perspective. While our visual system has developed a reliable strategy to decompose transparent motion signals, the extraction of transparent motion signals is still an extremely difficult and demanding task for artificial algorithms (Andersen et al.,1990; Braddick \& Qian, 2001). This is largely due to the use of spatial integration to improve signal-to-noise-ratios. This leads to a combination of different directional components into a single direction vector. Furthermore, most of the current models of physiological mechanisms for direction selectivity rely on applying inhibitory interactions between neurons, which code for different directions of motion. While this might be adequate for a single motion stimulus, this mechanism would lead to the silencing of neurons exposed to transparent motion and effectively lead to a diminished ability to perceive transparent motion patterns (Braddick \& Qian, 2001). As this is not the case and organisms are well able to distinguish overlaying surfaces of motion patterns, the natural system must process transparency in an alternative, more sufficient manner.

Cells which respond to visual motion are already present in the primary visual cortex. It could be shown however, that cells in V1 do not seem to be involved in the recognition of visual stimuli consisting of multiple motion directions. Snowden et al., 1990 could show that when presented with a transparent motion pattern containing preferred and null-direction of motion, V1 cells showed a response similar to when only the preferred direction was presented. Cells in area MT however, showed a different pattern of response. Their firing rate was suppressed by the presence of the null-direction pattern. Furthermore, Qian and colleagues 
(1994) tested different random dot displays and looked for correlates of the percept of these patterns in area V1 as well as MT. They showed that when bidirectional, overlaying stimuli were paired on a local base, the perception is that of a flicker, whereas when the two directions were decoupled, the stimulus was perceived as two separate surfaces. V1 cells could not distinguish these two different cases, whereas MT cells responded well to the decoupled surface. Both studies show that cells in V1 are less capable to segregate multiple motion components in a visual input. Qian and colleagues suggested that due to weak suppressive interactions between different directions of motion, neurons in V1 rather behave like unidirectional motion energy detectors signalling the presence of moving components in a pattern, regardless of the presence of other components. Thus, the response pattern of MT neurons correlated better with our perception of motion transparency than that in V1. Furthermore findings of other studies suggest that other components, such as binocular disparity and luminance, are integrated into the computational process in $\mathrm{MT}$, which contribute to the segregation of motion components (Bradley \& Andersen, 1998).

One assumption is that multiple motion patterns are represented by separate populations of direction-selective neurons. This would result in a population activity curve, which contains peaks of activity for each directional component. Treue et al (2000) recorded responses of MT neurons to bidirectional transparent motion stimuli, in which the angle of separation between the two motion components was varied. They showed that in order to encode the different directions, the population activity does not necessarily have to show two different peaks. The population activity was well described by a scaled sum of the response profiles to the individual motion components when these were presented alone. Due to the rather broad tuning curves of MT cells, the overlap of the different tuning profiles was large. The population response resulted in two separated peaks when the angle of separation exceeded 90 degrees. Psychophysical studies could show however, that transparent pattern can be distinguished if the angle between the two directions of motion is larger then 10 degrees (Groh et al., 1997). As a consequence, we must conclude that the perception of multiple directions within the same visual space does not rely on the most active neurons alone, but that an additional mechanism must be taken into account. This additional mechanism 
could come in the form of attention. Studies invastigating the motion aftereffect in transparent motion stimuli could show that attention alters the susceptibility to adaptation to transparent motion (see Snowden \& Verstraten, 1999 for review). Therefore, attention might be an additional tool for direction selective cells to segregate motion components (Braddick \& Qian, 2001).

In the last paragraph we discussed transparent motion stimuli and the role of area MT in their processing. Furthermore, we suggested that visual attention might contribute to the successful processing of those stimuli. In the next paragraph we will introduce the concept of visual attention and highlight the characteristics relevant to this thesis.

\subsection{Attention}

At any given time, our visual system is confronted with a vast amount of visual information about our surroundings. When reading these words for example, not only information about the individual letters enters the visual system through the retina, but also information about the desk, the walls of the room, the light outside the window, enters the same stream of processing. Despite the fact that the visual system takes up large parts of the brain (50\% in the macaque cortex, and $20-30 \%$ of the human cortex (Orban et al., 2004)), the computational resources are nevertheless limited. The brain is thus faced with the challenge of actively deciding from moment to moment which parts of this huge amount of information are behaviourally relevant and should be processed further. One key aspect by which the visual system is able to take this decision is visual attention.

Attention has been a subject of research for more then a century and the essence of attention is still today well captured by the words of William James in 1890: "Everyone knows what attention is. It is the taking possession by the mind, in clear and vivid form, of one out of what seem several simultaneously possible objects or trains of thought. Focalization, concentration, of consciousness are of its essence. It implies withdrawal from some things in order to deal effectively with others, and is a condition which has a real opposite in the confused, dazed, scatterbrained state which in French is called distraction, and Zerstreutheit in German" 
But despite the intuitive understanding of what attention is and what effects it has, we have not yet reached a full understanind of its fundamental mechanisms. Looking at the increase of publications in recent years (Raz \& Buhle, 2006), it is obvious that the topic has not lost its fascination.

The effects of attention have been well described and captured in a large amount of publications over the years. The effects range from an improvement in reaction time and accuracy, to enhancement in spatial selectivity and contrast, size and speed perception (e.g. Posner, 1980; Carrasco et al., 2004, Anton-Erxleben et al. 2007, Turatto et al., 2007; Carrasco, 2011, for review).

Furthermore, imaging techniques like fMRI have shown strong effects of spatial attention in striate and extra-striate cortex (e.g. Slotnick et al., 2003; Boynton, 2011, for review). We can go a step further and analyse local field potentials, which capture the reaction of a population of cells in one or more specific brain areas. It could be shown, that depending on the attentional state, the extent at which spiking events and the gamma-band frequency of local field potential correlated varied (Womelsdorf et al., 2007).

On the neuronal level, attentional effects can be observed as a difference in firing rate of a neuron depending on whether or not the subject attends to a certain stimulus. Given the same sensory input, the cell increases its firing rate to a stimulus when that stimulus is attended compared to when it is not attended. For a long time it was believed that attentional effects are a feature of higher areas of cortex. The finding of attentional modulation in area MT (Treue \& Maunsell,1996), which was till then believed to be primarily a sensory area and unaffected by higher cognitive processes, challenged this view. Nowadays modulatory effects have been even located even in the LGN (McAlonan et al., 2008). The strength of attentional modulation, however, increases along the cortical hierarchy (Maunsell \& Cook, 2002; Figure 3; see Carrasco, 2011, for review).

The magnitude and the mechanism of the attentional modulation are largely dependent on the type of attention executed. Different types of attention are defined, among them spatial attention (attentional modulation based on the relevant location), feature-based attention (attentional modulation based on an relevant feature) or object-based attention (attentional modulation is executed over 
several grouped features (object)). Feature-based and spatial attention are the two types of attention most relevant for our studies and we will therefore focus on these for the further discussion of this topic.

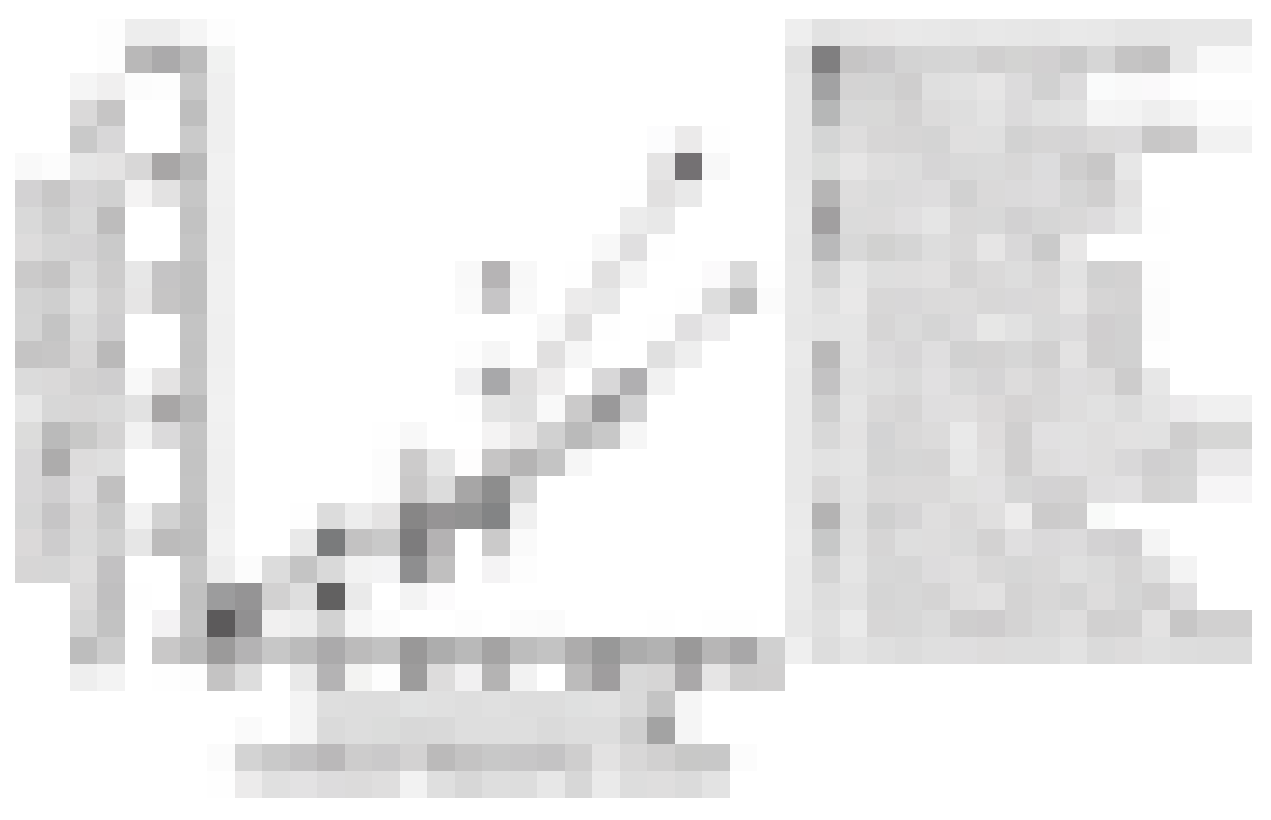

Figure 3 -Enhancement of attentional modulation along the cortical hierachy

Different symbols represent the different studies listed on the right. Lines in between symbols indicate data, which comes from the same study. (from Montijn (2012), with permission from R.J.A. van Wezel)

Spatial attention describes attentional modulations based on the spatial position of a relevant stimulus. Consider, for example, searching for a document on a cluttered computer desktop upon which the documents are spatially clustered according to topics. Knowing the topic, one would focus on the spatial location at which these documents were located in order to find the relevant document, ignoring other locations. Spatial attention helps us to detect behaviourally relevant stimuli at the cost of neglecting stimuli which fall outside this "spotlight of attention" (Posner, 1980). In psychophysical experiments it could be shown that the allocating the spatial focus prior to a presentation of visual stimuli benefits greatly behavioural performance by improving reaction times (Posner, 1980). On a neuronal level spatial attention alters the firing rate of neurons which process sensory information at the attended location. Cells in area V4 show for example an 
increase of $26 \%$ in their firing rate when the monkey is attending to a stimulus inside its receptive field, compared to when the monkey is attending to a stimulus placed outside the receptive field (McAdams \& Maunsell, 1999). The magnitude of the spatial attentional modulation is independent of the defining stimulus features. The effects of spatial attention have been shown for several different visual areas in the last years (Maunsell \& Cook, 2002, for review). Area MT for example shows on average $15 \%$ modulation of spatial attention (Treue \& Maunsell, 1996).

In most cases, spatial attention alters the neuronal responses by a multiplicative scaling of the firing rate (Maunsell \& McAdams, 2001). Attention does not influence a neurons selectivity, but its sensitivity. This effect is captured in the change of the tuning curve of a neuron. Attention modulates the firing rate along the tuning curve by the same releative magnitude, leading to a general, multiplicative enhancement of the neuron's sensitivity, but leaving the width of the tuning curve and with that, the neuron's selectivity unchanged. One exception to this rule is the influence of spatial attention on the structure of receptive fields. In 1995 Desimone and Duncan introduced their biased-competition model of attention. The model describes the competitive interactions when multiple stimuli are presented within one receptive field. It states that in the case of competition, the response of a neuron is modulated primarily by the attended stimulus. One implication of this model was that the receptive field of a neuron would close around a focus of attention, leading to shrinkage and a shift of the receptive field. Which is largely the same as changing the neurons selectivity on a spatial level. This mechanism, however, stands in contrast to the principle of multiplicative attentional modulation, which specifically states, that selectivity is unchanged by attention. Womelsdorf et al. (2006) could nevertheless show that cells in area MT shift the receptive fields depending on the spatial focus of attention. They placed two stimuli within the receptive field of an MT neuron and instructed the monkey to attend to either one of the two stimuli. While the monkey was attending they measured the extent of the receptive field by presenting a brief probe stimulus. They found that the receptive field shifted towards the attended location and also shrank around the focus of attention. The modulation of the receptive field structure represents a change in the selectivity of the neuron on a spatial level, which is an effect not in accordance with the mechanisms of multiplicative effects. 
The modulation of receptive field structures is one example from the extra-striate cortex for a non-multiplicative effect of spatial attention. In the striate cortex however, non-multiplicative effects seem to be the rule, rather then the exception. Attentional effects in the primary visual area were found in fMRI studies. In the late 90s three different studies could independently show robust spatial attentional modulation of fMRI responses (see Boynton (2011) for review). These findings were surprising as single-cell recordings had not found substantial modulations in V1 (Luck et al., 1997), Haenny \& Schiller, 1976)). However, the attentional effects in fMRI studies in V1 were reliably replicated and have been established (see Boynton (2011) for review). Furthermore, it could be shown that spatial attention shows strong modulatory effects even in the absence of a stimulus. The magnitude of these effects can be just as strong as in the presence of a stimulus over a wide range of contrasts (Murray, 2008). This result implies that modulatory effects of spatial attention do not follow the primary mechanism of multiplicative modulation as is the case for extra-striate areas. Recently, Thiele and colleagues showed in neurophysiological recordings that spatial attention had an additive rather then a multiplicative effect on the contrast-response function of V1 cells (2009). They tested the effects of spatial attention on the contrast-response function. They showed that once a stimulus becomes visible, the attentional modulation becomes contrast invariant. This stands in contrast with models of response-gain (McAdams \& Maunsell, 1999) or contrast-gain (Martinez-Trujillo \& Treue, 2002), which predict attentional effects to be contrast-dependent (see Reynolds \& Chelazzi, 2004, for review). Unlike spatial attentional effects in higher areas, Thiele and colleagues found that an additive model that assumes a constant modulation factor over all contrast values best described their effects.

The second type of attention, which we want to discuss in more detail, is featurebased attention. Unlike spatial attention, feature-based attention alters neuronal responses depending on the feature of a stimulus rather then on the spatial location. Going back to the example of the cluttered desktop from before; we might consider looking for a document about which we cannot remember where on the desktop we had put it, only that it was specially tagged by colouring the document's name red. Therefore our attention will not be focused on specific locations on the desktop, but on the feature 'red' in order to find the document we 
are looking for. As this example shows, feature-based attention is particularly helpful in visual search paradigms, when targets can be identified with knowledge about the target's parameters, e.g. colour (Treisman \& Gelade,1980).

Feature-based attention effects have been demonstrated in a range of neurophysiological studies (Maunsell \& Treue, 2007). In area MT, for example, it could be shown that when two stimuli are presented on the screen, one inside the receptive field and one outside the receptive field, and the monkey attends to the stimulus outside the receptive field of a cell, the cell's firing rate nevertheless gets manipulated depending on whether the direction of the attended stimulus outside the receptive field was the preferred or non-preferred direction of the cell (Martinez-Trujillo \& Treue, 1999). For MT the firing rate increases by $13 \%$ when the monkey attends to the preferred direction as compared to the non-preferred direction. Feature-attention effects for area V4 range in the same magnitude (McAdams\&Maunsell 2000). Effects of feature-based attention have also been found in V1 for studies using fMRI (Saenz et al., 2002). The feature modulation for single cells however, has still to be shown (Boynton, 2011).

The finding of the feature-based attentional modulation led to the proposal of the feature-similarity gain model (Treue \& Martınez-Trujillo, 1999). The model proposes a multiplicative modulation by attention, in which the sign and the magnitude of the modulation represent the similarity of the attended stimulus feature and the neuron's preferred feature. The model incorporates also spatialbased effects, because it allows space to be treated as a feature of a stimulus. The modulation in firing rate depending on whether attention is directed inside or outside the receptive field can therefore also be interpreted as a modulation caused by the low similarity between the attended and the neuron's preferred spatial feature. The feature-similarity gain model also accounts for effects of attention when no stimulus was actually present (Maunsell \& Treue, 2006). The modulation it describes is a gain change in which the magnitude of the modulation is determined by its sign. Therefore the effect is independent of the stimulus, which drives the neuron.

Recently, the feature-similarity gain model has been incorporated into the "normalization model of attention" (Reynolds \& Heeger, 2009). Apart from the aforementioned biased-competion model and feature-similarity gain model, response-gain (neuron fires more when stimulus is attended than unattended, 
(e.g. McAdams \& Maunsell,1999) and contrast-gain (the response modulation by attention can be described in the same way as if the stimulus contrast would increase (Martinez-Trujillo \& Treue, 2002) have been prominent models to capture the essence of attentional effects in the extra-striate cortex. The recently proposed normalization model of attention elegantly incorporates standing concepts and understanding of attention into one theoretical framework. The normalization model of attention combines the visual input (stimulus drive) with the modulatory effect of attention (represented as an "attentional field") to produce an excitatory drive. Attending to one of two stimuli therefore will lead to an enhancement of the responses of the neurons, which are tuned to the attended stimulus. Simultaneously, the model assumes a "suppressive drive", which arises from the excitatory drive in combination with a suppressive field (which represents lateral inhibition). The final population response depends on the orientation and the receptive field location and size. It is calculated by dividing the excitatory drive by the suppressive drive. The purpose of the division is the normalization of the response magnitude of individual neurons to that of the population as a whole (Reynolds \& Heeger, 2009, Montijn et al., 2012). The model has been proven to capture a lot of attentional effects very precisely, such as gain changes in orientation or direction tuning curves (McAdams \& Maunsell, 1999; Treue \& Martinez-Trujilo, 1999). The principle of normalization appears to be a promising candidate to describe attentional mechanisms in the cortex (Carandini \& Heeger, 2012).

In the last chapter we reviewed some of the basic aspects which are important theoretical concepts to the following chapter of the thesis. However, we will discuss certain aspects of the aforementioned topics of the visual processing of motion and the influence of attention in more details in the introductions to each study. 


\section{Original manuscripts}

This chapter contains the following manuscripts:

2.1 Attentional modulation of neuronal response in macaque area MT to transparent motion patterns. Stephan VM, Lochte A, Kozyrev V, Veith V, Treue S; prepared for submission.

Author contribution: TS, LA and KV designed the original experiment. LA implemented the experiment. LA and SVM trained the animals on the task; LA, SVM and VV and collected the data; SVM and KV analyzed the data. VSM and LA wrote the manuscript; TS edited the manuscript. All authors discussed the results and commented on the manuscript.

2.2 Spatial attentional modulation of multi-unit activity in macaque V1. Stephan VM, Liu R-F, Treue S; prepared for submission

Author contribution: SVM designed and implemented the original experiment. SVM implemented the new recording technique. SVM trained the animal on the task and collected the data. SVM and RFL analyzed the data. SVM wrote the manuscript. TS edited the manuscript. All authors discussed the results and commented on the manuscript. 


\subsection{Attentional modulation of neuronal response in macaque area MT to transparent motion patterns}

The behavioral and neurophysiological consequences of directing visual attention to an isolated stimulus in the receptive field (RF) of an individual neuron are well described in the literature. Further, studies with two stimuli in the RF showed stronger attentional modulations than measurements made using single RF stimuli. While few information about the effects of attention on spatially separated stimuli within one RF is available, up to now no neurophysiological study succeeded in investigating the attentional modulation of the population activity profile of transparent motion stimuli on a single-unit level. Motion transparency is defined by moving elements, grouped together to give the impression of overlapping surfaces, which provides an important challenge to models of motion perception.

For this study, two macaque monkeys were trained on the challenging task to selectively attend to one direction component of transparent motion patterns. We examined the influence of endogenous attention on the processing of transparent motion by recording extracellular activity from individual neurons in the macaque area MT. We characterized the modulation of spatial and feature-based attention as well as their combinatory influence on the population activity. Additionally, we draw comparisons with the findings in our complementary study on spatially separated motion components (Kozyrev et al., prepared for submission) 



\title{
Attentional modulation of neuronal response in macaque area MT to transparent motion patterns
}

\author{
Valeska M. Stephan ${ }^{1,2}$ \& Anja Lochte ${ }^{1,2}$, Vladislav Kozyrev ${ }^{3}$, \\ Vera Veith ${ }^{1} \&$ Stefan Treue $e^{1,2}$
}

\begin{abstract}
${ }^{1}$ Cognitive Neuroscience Laboratory, German Primate Center, Göttingen, Germany

${ }^{2}$ Bernstein Center for Computational Neuroscience, Göttingen, Germany

${ }^{3}$ Real-time optical imaging lab, Ruhr-Universität Bochum, Bochum, Germany
\end{abstract}

\begin{abstract}
Transparent motion perception requires the distinction of multiple motion components within the same part of visual space. This study investigates the influence of spatial and feature-based attention on the representation of the direction components of transparent motion by recording responses from neurons in the middle temporal area (MT) of two macaque monkeys. The stimuli consisted of two overlapping random dot patterns (RDPs), moving within a stationary aperture as a transparent pattern. One transparent pattern was positioned within the receptive field (RF), while a physically identical stimulus was presented in the opposite hemifield. The relative angle of 120 degrees between the component directions was kept constant. By systematically varying the overall pattern direction, neuronal responses to 12 different RDP directions were measured. We employed three behavioral conditions with otherwise identical stimulus conditions where attention was directed to one of two motion components of the transparent pattern inside (attend-in) and outside (attend-out) the RF as well as to the fixation point (attend-fix).

The population activity profile was well fit by the sum of two Gaussians, showing two peaks corresponding to the two stimulus configurations in which one of the directions moved in the neuron's preferred direction. The activity of the population was upregulated when attention was shifted from the fixation point to the stimulus
\end{abstract}


inside the receptive field. We also observe an upregulation of the same magnitude when attention was allocated to the stimulus outside the receptive field. This is particular interesting. Unlike other studies, we do not find an enhancement or a suppression depending on the attended feature when attending to a stimulus outside the receptive field. We observe an enhancement in the activity for conditions in which the preferred feature is attended, as well as in conditions in which the non-preferred feature is attended.

\section{Introduction}

Attention is an eminent and efficient mechanism for selecting information for prioritized processing at the expense of unattended aspects, dependent on behavioral relevance. Thereby, a rational use of the energetic cost of cortical computation is assured. Visual attention has been shown to result in a better task performance, shortened reaction times (Posner et al., 1980; Carrasco et al., 2004, 2006) and a general enhanced visual representation of various stimulus attributes, like increased apparent speed (Turatto, 2007) and size (Anton-Erxleben et al., 2007). Several physiological studies demonstrated the neuronal correlate of attentional modulation to be an increase in firing rates of cortical sensory neurons (Yantis \& Serences, 2003; Reynolds \& Chelazzi, 2004; Maunsell \& Treue, 2006). Studies on attentional modulations when presenting a single stimulus inside the RF suggest that neuronal selectivities are not fundamentally altered by attention (Salinas \& Abbott, 1997; McAdams \& Maunsell, 1999; Treue \& Martinez-Trujillo, 1999). A general way for describing the effects of attentional modulation qualitatively is the feature-similarity gain model (Martinez-Trujillo \& Treue, 2004; Treue \& Martinez-Trujillo, 1999). According to this model, the sign and magnitude of attentional modulation of a particular neuron are predicted by the similarity between the preference of the given neuron for a particular feature (direction, color as well as a spatial location) and the attended feature.

Most electrophysiological studies focused on the attentional influence on a single stimulus inside the RF. However, in real life situations the visual system is often confronted with much more complex patterns. Experimentally, such a case was mostly reproduced by placing multiple stimuli, differing in one stimulus dimension, such as orientation, within the RF. In studies presenting two stimuli in one RF, 
mostly spatial-based attention effects play a role that enhance the modulation by performing a weighted average that can lead to a shift of the center of weight for a RF toward the attended stimulus (Connor et al., 1996; Connor et al., 1997; Womelsdorf et al., 2006; Womelsdorf et al., 2008; Anton-Erxleben et al., 2009). When attention is redirected between two spatially coincident stimuli inside the RF, changes in the RF profile cannot contribute to the perceptual segregation of such patterns. In this case, feature-based mechanisms are allocated.

It has been shown that attending to a single stimulus in the RF of a single neuron leads to a moderate enhancement of its response. However, responses are more strongly modulated when attention is shifted between a relatively weak stimulus and the neuron's preferred stimulus, both spatially segregated within one RF (Moran \& Desimone, 1985; Treue \& Maunsell, 1996; Luck et al., 1997; Reynolds et al., 1999; Ghose \& Maunsell, 2008; Lee \& Maunsell, 2010). In these studies, the response to a preferred and a non-preferred stimulus were reported to be a scaled average of the responses to the individual stimuli when presented alone. With such a stimulus design the push-pull effect of attention (Treue, 2001) is evident in a response enhancement when the preferred stimulus is attended (push component) and a reduced response strength if the non-preferred stimulus is attended (pull component), compared to the sensory response.

Hereby, the question arises how MT neurons encode different motion vectors that are transparently presented within their RF. A difference between overlapping and non-overlapping stimuli inside a RF would only lead to different neuronal responses in the absence of a simple pooling of all inputs. Majaj et al. (2007) recorded the response of neurons in MT to plaids whose component gratings overlapped within a patch. These were compared to responses to the same component gratings presented in separate patches, showing that cells selective for the motion direction of the whole pattern in the overlapping gratings lost this selectivity when the gratings were separated. Instead they became selective for the individual motion component direction. This suggests, that the computation of pattern-direction selectivity in MT is done locally on a smaller scale than the whole RF and not by a simple pooling of the inputs. To understand the underlying mechanism, it is important to qualitatively measure MT's neuronal response to transparently overlapping stimuli. 
Among questions concerning the computation of multiple-motion components in one RF, transparent motion stimuli depict a particular complexity, as they require the extraction and encoding of several stimulus properties at a given spatial location, whereas local motion cues have to be assigned correctly (Snowden \& Verstraten, 1999). The neuronal responses of the middle temporal visual area (MT) to unattended transparent motion patterns of different separation angles were investigated by Treue et al. (2000). The population response to two widelyspaced motion components showed the presence of two peaks corresponding to configurations when one of the two patterns moved in the preferred direction of the neuron. This is in line with the assumption that the profile of responses to motion in multiple directions is the scaled sum of the responses to the individual components. Thus, a linear combination of the individual components seems to be sufficient for the encoding and the decoding of transparent stimuli.

However, does attention on the transparent stimuli act by simply altering the weight of the individual components? Not much is known about the effects of attention on transparently overlapping stimuli. Patzwahl \& Treue (2009) found a mean response modulation of around $32 \%$ for shifts of attention from the antipreferred to the preferred direction of transparent random dot patterns in area MT. This study used stimuli of different colors to ease the perceptual separation and merely reported the extreme cases of the neuron's preferred and anti-preferred direction.

While attention is a predestined mechanism to disentangle transparent motion patterns, the exact mechanism by which the neural responses to stimuli of completely overlapping and iso-colored direction components is modulated by attention remains elusive.

Here we investigated the modulation of neuronal population responses to transparent random dot motion patterns by attention by comparing conditions where spatial and feature-based attention work in isolation or in concert. We found an attentional enhancement when directing spatial attention into the receptive field but no attentional modulation based on the attended feature. 


\section{Materials \& Methods}

\subsection{Experimental Procedures}

We recorded the responses of direction-selective neurons in area MT of two male macaque monkeys (Macaca mulatta) to moving transparent random dot patterns (RDPs) while the animals performed a visual attention task. The animals were implanted with a custom made implant to prevent head movements during training and recording, and a recording chamber whose position was based on anatomical MRI scans. Surgeries were performed aseptically under isoflurane anesthesia, using standard techniques. All experiments reported in this study were approved by the local animal research committee and complied with relevant laws.

\subsection{Apparatus}

The experiments were conducted in a dimly lit cabin. Animals were seated in a custom made primate chair and viewed the display binocularly from a distance of $57 \mathrm{~cm}$. Visual stimuli were presented on a CRT monitor (LaCie, Electron22 Blue IV) with a display resolution of 40 pixels per degree and a refresh rate of $75 \mathrm{~Hz}$. The eye position was monitored with a high-speed video-based eye tracker at a sampling rate of $230 \mathrm{~Hz}$ (ET-49, Thomas Recording, Giessen, Germany). Stimulus presentation, reward control, monitoring of eye position and collection of behavioral and electrophysiological data was controlled by custom-made software run on an Apple Macintosh PowerPC.

\subsection{Recordings}

Neuronal extracellular activity was recorded from direction-selective MT neurons. In total we recorded 84 complete datasets from both monkeys (monkey $\mathrm{P}=49$ cells, monkey $T=35$ ). The cells were determined to be in area MT by their anatomical position (the chamber was non-ferromagnetic, so it was possible to verify the locations of our recording sites using additional postoperative MRI scans) and their physiological characteristics (directional selectivity, RF size and position). Recordings were performed either with a single tungsten microelectrodes (FHC Inc., Bowdoinham, ME, USA) or a 3-channel system, using quartz-tungsten microelectrodes (The Mini Matrix System, Thomas Recording, Giessen Germany). After the penetration of the dura mater with a sharp guidetube, 
a hydraulic micropositioner (single electrode; David Kopf Instruments, Tujunga, CA, USA) or a rubber tube drive (3-channel system) was used to advance the electrode. Impedances ranged from 0.5 - $4 \mathrm{M} \Omega$. Neural data was recorded and sorted online using the MAP data acquisition system (Plexon Inc., Dallas, TX, USA). Data were filtered (frequency range $150 \mathrm{~Hz}-5 \mathrm{kHz}$ ) and amplified (gain range 1000 - 32000).

\subsection{Stimuli}

We used RDPs consistent of small bright dots (density: 10 dots per deg$^{2}$, luminance $85 \mathrm{~cd} / \mathrm{m}^{2}$ ) plotted within a stationary circular virtual aperture on a gray background (luminance $15 \mathrm{~cd} / \mathrm{m}^{2}$ ). The aperture was sized and positioned to fit within the classical receptive field of the neuron under study as determined by an initial manual mapping procedure. For uni-directional cue patterns, all dots moved coherently in the same direction; for transparent stimuli, half of the dots were assigned to each direction.

\subsection{Behavioral task}

The animals were trained to attend to one of two transparently overlapping moving RDPs in the presence of a transparent RDP of the same configuration in the opposite hemifield. The behavioral paradigm is depicted in Fig. 1. Monkeys started a trial by touching a lever and responded by releasing it. Every trial started with the presentation of the fixation point. In the attend-in and attend-out conditions, a moving unidirectional RDP (the cue) was presented after $225 \mathrm{~ms}$ of fixating. The cue moved for $500 \mathrm{~ms}$, indicating the location (either inside the RF or in the opposite hemifield) and the direction of motion that the animals were later instructed to attend to. A blank period of $800 \mathrm{~ms}$ followed, during which only the fixation point was visible on the screen. In the subsequent attentional period, two transparent stimuli whose component directions had a constant relative angle of $120^{\circ}$ were presented for $400-4600 \mathrm{~ms}$. The directions of the two motion components were systematically varied in steps of 30 degrees. One of the stimuli was placed inside the RF, the other was positioned at equal eccentricity in the opposite hemifield. The animal's task was to attend to the cued RDP and to respond to a doubling of speed in this surface within a reaction time window of 150-500 ms. Any of the three remaining distractor surfaces could change speed 
up to three times. Trials in which only distractors but not the target changed their direction were rewarded after the end of a trial, if the monkey did not respond. Trials in which the animal broke fixation or responded outside the reaction time window were considered as errors and were aborted without reward. In a sensory condition (attend-fix), the monkeys were asked to respond to a luminance change (from 85 to $52 \mathrm{~cd} / \mathrm{m}^{2}$ ) occurring on the fixation point at a randomized time point between 800 and $2400 \mathrm{~ms}$. The transparent patterns were also presented in this condition and had to be ignored. The different attentional conditions and direction combinations were randomly interleaved within the experiment.

$<$ Insert fig.1 approximately here>

Throughout a trial, the animal's gaze had to be maintained on a fixation point within $1^{\circ}-1.25^{\circ}$ of visual angle. The monkeys broke their fixation in $24 \%$ of the trials. In $55 \%$ of the trials, they performed correctly and in $20 \%$ of trials they responded outside the reaction time window.

\subsection{Data Analysis}

Data were analysed offline using custom scripts written in Matlab (The MathWorks, Inc.). For the analysis of neuronal data, only correctly completed trials were included. Firing rates for each condition were calculated by averaging the spike density functions in a time window of 200 to $700 \mathrm{~ms}$ after the onset of the transparent RDPs. Responses were averaged across trials and normalized to the highest firing rate in the attend-fix condition. Population responses were computed by averaging point-by-point across the normalized neuronal responses and were aligned regarding the preferred direction that was determined by obtaining a tuning curve from the responses to the cue period (50-500 ms after cue onset, see Fig. 2 \& 4 "single-fix" condition). Errors given are standard errors of the mean calculated across cells. For each recorded neuron, the attentional index (AI) was computed, defined as the difference in firing rates between two conditions, divided by their sum (e.g. $A l=\left(f r_{\text {In }}-f r_{\text {Out }}\right) /\left(f r_{\text {In }}+f_{r_{\text {Out }}}\right)$, where $f r_{\text {In }}$ and $f_{\text {Out }}\left(f r_{\text {Fix }}\right.$ respectively) are responses when attention was directed inside and outside the $\mathrm{RF}$ or to the fixation point). 
To quantitatively estimate the attentional alteration of the tuning curves across different conditions, the data set for each neuron was fitted by a set of periodic Gaussian functions using the MATLAB Fitting Toolbox. The data from transparent motion conditions were fitted by the sum of two Gaussians corresponding to the independent responses to the two RDPs with independent across conditions variation of all seven parameters. Attentional indices were defined as described above with respective fitting parameters in the attention conditions used instead of firing rates.

We recorded 84 complete datasets in total. After a preselection, 44 cells were included into our final analysis (Monkey $P=23$, Monkey $T=21$ ). The preselection criteria were fits to the response profiles in the single-fix condition and to the sensory condition (attend-fix, Fig. 2 \& 3) with $r^{2}$-values equal or larger than 0.79 . To ensure that the attend-fix condition was free of modulatory effects, we calculated the differences in the height of the two peaks. Because attention is not deployed to any of the presented stimuli in this condition, we expect to observe a bimodal tuning curve, in which the two peaks indicating the preferred direction are of almost equal height. For our selection the peak height difference had to be < $30 \%$.

In order to ensure the reliability of the applied fitting procedure, parameters of both Gaussians in each of the two conditions should be significant. We checked the $95 \%$ confidence intervals of the Gaussian amplitudes in the attend-fix condition. If at least one of them included zero (indicating that the response did not modulate as a function of stimulus direction), the whole data set was excluded. This was the case for 12 . The remaining subset of 32 cells showed a clear bilobed tuning in the attend-fix condition. On the other hand, a non-significant Gaussian in the attend-in or attend-out condition was not a reason for excluding the data set, since switching of attention might suppress one of two components. In such cases, refitting of the attend-In tuning curve was performed, keeping the location and standard deviation of the non-significant Gaussian fixed at the respective values inherited from the attend-fix condition fit. Thereby, our procedure led to a conservative estimation of the indices in ambiguous cases.

The analysis based on fitting parameters was performed in the subpopulation of 32 neurons. Distribution of the values of the fitted parameters for the attend-fix, 
attend-in and attend-out data within the subpopulation is depicted in the supplementary Fig. $\mathbf{S 2}$.

\section{Results}

We analysed the neuronal responses of 44 direction-selective neurons in area MT of two macaque monkeys to moving transparent motion RDPs. One transparent random dot motion pattern was positioned inside the RF of the neuron under study within a stationary virtual aperture. A second transparent RDP, which was physically identical to the inside pattern, was simultaneously shown at the opposite side of the visual field. The stimuli had a relative angle of 120 degrees between the component directions. By systematically varying the overall pattern direction, neuronal responses to 12 different RDP direction combinations were measured. An initial unidirectional cue indicated the relevant spatial location and direction of motion in a given trial. While maintaining gaze on a fixation point, the animals were instructed to respond to a speed increment within the cued direction. The cued direction could either be located inside the RF (attend-in condition) or in the aperture in the opposite hemifield (attend-out condition). In a sensory condition (attend-fix condition), the monkeys were asked to respond to a luminance change occurring on the fixation point, while ignoring the transparent patterns.

The tuning curves with attentional indices for the three conditions of an example neuron are shown in Fig. 2. Additionally we show the single-fix condition. Our population of 44 neurons had a median $r^{2}$-value for the single-fix condition of 0.94 and for the attend-fix condtion of 0.93 . The mean peak height difference was $13.5 \%$.

<Insert fig.2 approximately here>

The actual activity results in a bimodal tuning curve with peaks of equal height centered around the stimulus configurations, where a preferred direction component was present. This finding matches the results of Treue et al. (2000) that predict that the scaled sum of the responses to the individual components of the transparent motion stimuli results in two peaks for transparent stimuli 
separated by more than $90^{\circ}$. In contrast, the single-fix curve depicts the response of the neuron, when only a single direction is presented within its receptive field. Consequently, the curve shows only a single peak according to the neuron's preferred direction.

When comparing the attend-fix tuning curve to the conditions, where attention was allocated to one of the two directions, the attentional modulation of the activity profiles to the physically identical stimuli is revealed.

$<$ Insert fig.3 approximately here>

To quantify the attentional modulation of the population activity by spatial and feature-based attention we calculated the attentional index for the complete population of 44 cells. When attention is switched from a moving transparent RDP inside the RF to a stimulus of identical configuration outside the RF, a purely spatial change in the attentional allocation happens, while feature-based attention remains unchanged. For the population of 44 cells the respective measurement is presented in Fig. 3. We did not find a significant modulation by spatial attention for our population of cells. However, when evaluating the modulation by featurebased attention by comparing the condition when the stimulus is attended outside the RF to the attend-fix condition, in which the stimuli are not attend, we find a significant average modulation of $17 \%$.

<Insert fig.4 approximately here>

As stated above, the three tuning curves derived from the responses to the transparent stimuli showed two peaks corresponding to the response components evoked by each of the two stimulus components. Without making any specific assumptions on the rules of the components' summation, such bilobed curves can be mathematically described by the sum of two independent Gauss functions (Fig. 4a; for details see Supplementary methods). We fitted the responses in each of three attentional conditions by the sums of two Gaussians (Fig. 4b).

To test our prediction for the entire sample of 44 neurons each cell's activity profile was aligned to the cell's preferred direction (determined from the cue period; see 
Materials \& Methods) and normalized to the highest firing rate in the fixation condition before averaging all individual profiles (upper panels in Fig. 5a, 5b, 5c). The depicted population activity in all three conditions shows a bimodal profile with the two peaks not being significantly different from each other in height (paired ttest, all $p$-values $p>0.05$ ).

To isolate spatial and feature-based attention effects, we separately compared the population activity profiles for two conditions respectively. Fig. 5a shows the comparison that reveals the modulation by the combination of spatial and featurebased attention, given by the attend fix vs. attend in relation. The comparison between attend in vs. attend out gives the spatial attentional modulation (Fig. 5b) and the attend out vs. attend fix the modulation by feature-based attention (Fig. 5c).

To test for the relative modulation across attentional conditions, we calculated the attentional modulation index between the fitted peak heights of each individual tuning curve for each pair of attentional conditions (histograms inserted in the top panels). Indices significantly different from zero would reveal that the modulation of the two peaks within one attentional condition is not of the same magnitude. This was not the case for our data sets (ttest, all p-values $>0.05$ ), indicating that the peak heights did not differ in any of our conditions.

The histograms in the middle panel show the distribution of changes of the gain of the Gaussians corresponding to when the preferred direction is attended (histogram on the right) as to when the preferred direction is not unattended (histogram on the left). The histograms are calculated based on the fits considering all seven parameters in the subpopulation of 32 neurons.

For the comparison of attend-in against attend-fix, the histogram for the modulation of the Gaussian representing the attended component of the transparent motion stimulus (histogram right, middle panel Fig. 5a) reveals an average attentional enhancement of $28 \%(p<0.01)$. If attention is directed from the fixation point to the non-preferred direction (histogram left, middle panel Fig. 5a) we find an average attentional enhancement in the same order of magnitude $(29 \%, p<0.01)$. The two populations of the parameters are not significantly different $(p>0.05)$. We observe a similar effect when comparing the attend-fix condition against the attend-out condition (histograms, middle panel Fig. 5c). In the feature-based attentional condition, both parameters of the Gaussian are 
upregulated as well. The right histogram in the middle panel of figure $5 \mathrm{c}$ shows an attentional enhancement of $27 \%(p<0.01)$ for the Gaussian representing the attended preferred direction in the spatial attentional condition. The left histogram depicts the same amount of enhancement for the Gaussian representing the nonpreferred direction $(27 \%, \mathrm{p}<0.01)$. The attend-out against attend-in comparison reveals the impact of spatial attention. However, we do not find any significant modulation in the calculated parameters when comparing those two conditions (Fig. 5b, Fig. S5b).

The lower panels in the figures show the attentional modulation indices, averaged point-by-point across the cells for the three comparisons. We find generally positive modulations in the case of feature-based attention and feature-/spatial attention. For pure spatial attention, we generally find low or no attentional modulation.

$<$ Insert fig.5 approximately here $>$

\section{Discussion}

In this study we investigate the influence of attention on the neural representation of multi-directional spatially overlapping motion patterns. Such "transparent motion" stimuli evoke a bimodal population activity profile. Keeping the physical stimulus conditions identical, we implemented three behavioural conditions, where attention was shifted inside (attend-in) and outside (attend-out) the RF as well as to the fixation point (attend-fix). By comparing the three conditions, we can analyse the effects of spatial attention, feature-based attention and the combination of those two. In contrast to studies, in which stimuli are spatially separated, our design also allows for an analysis of the relative contribution of attended versus unattended features at the same spatial position.

\subsection{The influence of attention on the processing of transparent motion}

The activity profile of one population of 44 MT neurons showed two peaks corresponding to the two stimulus configurations in which one of the directions moved in the neuron's preferred direction. In the absence of attentional allocation 
to the stimuli the two lobes are of equal height, reflecting their identical sensory weights. The individual tuning curves can be well approximated by the scaled sum of the responses to the individual motion components (Treue et al., 2000). This allows us to estimate the effect of various types of attention on the two motion components.

The population activity profile can be well fit by the sum of two Gaussians, enabling a quantitative comparison of neuronal responses for the attend-in versus attend-out conditions and the attended conditions versus the sensory condition (attend-fix). For feature-based attention and the combination of feature- and spatial attention we find an enhancement of the population response to the attended direction, as well as to the unattended direction (Fig. 5 a,c, Fig. S5 a,c.). In accordance to those findings we do not see any significant differences in the ratio of the peak heights within conditions. Therefore the response to the attended non-preferred direction is modulated by the same magnitude as response to the attended preferred direction. Furthermore we do not find a difference in the magnitude of modulation between the responses in the attend-in and attend-out conditions (spatial attention). This indicates that attentional modulation in our study is independent of the spatial allocation of attention, but solely based on a feature effect. Global effects of feature-based attention are described by the feature-similarity gain model, which states that if a neuronal response is modulated by attending to a specific feature, the modulation is independent of the spatial position of that attended feature (Treue \& Martinez-Trujillo, 1999). If the attended feature is positioned outside of a neurons receptive field, the neuronal response will nevertheless be modulated by the attended feature. The sign and magnitude of the modulation is dependent on the feature; if the feature matches the preferred feature of the neuron, the modulation will be an enhancement, while a non-preferred feature will lead to a suppression. In our case we do find an effect of an attended feature which is positioned outside the receptive field of a neuron. However, this modulation does not follow the predictions of the feature similarity gain model, as we do not find any suppression. The neuronal responses are always enhanced with the same magnitude. The observed modulation is comparable with the multiplicative modulation found in spatial attention (Maunsell \&McAdams, 2001). 
4.2. Comparison to studies on attentional modulation of separated motion components in one RF

In a parallel study (Kozyrev et al., prepared for submission) we collected data in a paradigm where two stimuli were placed within the RF of MT neurons, similar to the study presented here. The difference was that the two motion apertures did not overlap, but were placed adjacently within the RF. This study did not investigate the attentional modulation of the full tuning curve for the attend-out condition.

In that study, an attentional shift from the fixation point to the preferred direction component inside the RF resulted in a $14 \%$ enhancement of the population's response and a suppression of $10 \%$ when the nonpreferred direction was attended. Consequently, they also found a modulation of $26 \%$ when calculating the attentional modulation index between the fitted peak heights. Both these outcomes are in contrast to our results. We did not find any differences in the magnitude of the attentional modulations for the different attended directions (middle panels, Fig. 5), nor did we find a significant difference in the modulation of the fitted peak heights (histogram in upper panels, Fig. 5).

Other than in our transparent motion study, in this experiment, mainly spatial attention was sufficient to solve the task. The consequent allocation of distinct types of attention might account for differences in the resulting modulations.

Furthermore, Kozyrev et al. found a broadening of $16 \%$ of the peak corresponding to the stimulus combination where attention was applied to the preferred direction inside the RF was found. The widening of the peak leads to a reduced selectivity for the attended direction, so that the flanks of the peak are most informative. We do not find any changes in the width of the tuning curves in our study.

<Insert fig.6 approximately here>

Several differences between both studies can be suggested as the basis of the discrepancy of the findings. A largely simplified processing mechanism within the two studies is depicted in Fig. 6. Here, a schematic overview of the V1 inputs to MT within the tasks when spatially separated apertures and transparent motion stimuli were positioned within an MT RF is given. On the level of V1, neuron 
populations encoding the different motion components are well segregated across the visual space when the motion components are spatially separated. However, in the transparent motion study, the very same V1 neurons receive input from both motion components. Such a more uniform input to area MT might contribute to differences found across the two studies.

Unlike for cells in area V1, within area MT no systematic difference in the representation of the two spatially non-overlapping stimuli might be expected. Still, both apertures did not seem to be equally represented, as in the fixation condition both peaks in the population activity profile should be of equal height if this was the case (data not shown). A simple reason for this finding is a potential systematic misplacing of the stimuli within the RF.

Attention acts as a filter mechanism that occurs most strongly at the level of the RF. This is compatible with the finding of previous studies demonstrating that selective attention to a stimulus causes a RF to shrink around the attended stimulus, thereby underrepresenting the unattended stimuli at nearby locations within or outside the RF (Womelsdorf et al., 2006; Anton-Erxleben et al., 2009). Thereby, the aperture containing the unattended direction component would be less represented and thus receive less attentional resources. Such a spatially biased representation of the two directions in the receptive field cannot occur in our transparent motion stimuli. Our data is in accordance with this notion. Presented and/or attended features are modulated with the same order of magnitude, which would not be the case if the stimuli would be represented with a spatial bias.

\subsection{Influence of task demand}

Part of the reason for the variability between the different findings in the above mentioned studies investigating attentional modulation of multiple motion stimuli might be the fact that the different tasks require a different amount of attentional load (Spitzer et al., 1988; Spitzer and Richmond, 1991). The impact of attentional modulation may depend on the perceptual load of the behavioural task, with more processing resources recruited when a higher attentional capacity is demanded. 
Chen et al. (2008) showed that task difficulty modulates the activity of neurons in $\mathrm{V} 1$ and that attentional gain can be enhanced by a factor of about 3 when task difficulty was increased. The greater attentional load caused by attending to one component of a transparent pattern (Fig. 5) could account for much of the difference that has been found in comparable studies (Suzuki \& Watanabe, 2009). In our task, the stimuli were iso-colored, making the task even more difficult, ensuring that the animals really attended the cued direction and not the color of the pattern. This was different in studies by Wannig et al. (2007) and Patzwahl (2009), where stimuli of different colors were used to ease the perceptual separation and thus reducing the required attentional load (Croner \& Albright, 1999). Correspondingly, Wannig et al., found a smaller attentional effect when using monochromatic stimuli as compared to heterochromatic stimuli.

Lee \& Maunsell (2010) used a task design, where attentional load was kept constant across different stimulus configurations. They found a strong modulation when more than one stimulus was presented within the RF and claimed that the broad range of attentional modulation effects seen across different conditions could be explained by a response normalization mechanism (Lee \& Maunsell, 2009).

In summary we find a strong effect of feature-based attention and no effect of spatial attention. The effect of feature-based attention acts on a spatiallyindependent, global scale, as predicted by the feature-similarity gain model. However, the modulation profile does not follow the prediction of the model. We do not find suppression of non-preferred features, but neuronal responses are always enhanced with the same magnitude, independent of the characteristics of the attended feature. Because the observed modulation mechanism does not distinguish between different features, it does not seem sufficient to separate the motion signals of different surfaces perceptually. For distinguishing different features independent of the spatial position, as it is necessary for perceiving a transparent motion stimulus correctly, the feature-based attentional mechanism would have to show a feature-dependent modulation. This feature-dependence, however, is absent in our current results. 
Recently it was suggested, that area FST, which is closely positioned and interconnected to area MT and MST in the extrastriate visual cortex, might be a visual area specialized in the segmentation of transparent motion stimuli, as the cells in FST do not show any effects of motion opponency (Rosenberg et al., 2008). More specificly feature-based attention effects might be found here. This notion however, implies an effect of feature-based attention, which is depending on the role of a visual area in the perception of a specific stimulus, where the effect of feature-based attention is most prominent the more closely a stimulus matches the preferred feature of a cell. Transparent stimuli might be too far from the optimal stimulus of a MT neuron to induce the required feature-dependent feature-based effect, which would be needed to perceptually separate a transparent motion stimulus. These suggestions are speculative and our data does not allow us to test this hypothesis.

The similarity of the tuning curves for the attend-in and attend-out condition might reflect the animals' behaviour. To produce such similar tuning curves the animal would have to behave the same in every trial, independent of the attentional condition. The most likely behaviour to achieve this would be to equally split attention between the two stimuli. This would lead to an attentional modulation, which would affect the two attentional conditions in an equal manner, just as we observe in our data. Alternatively it might be argued that the animal shifts its attention in the course of the trial. Due to the complexity of the task, the animal might need to attend to the complete stimulus arrangement in the beginning of the trial to gather enough information in order to solve the task and only later focuses its attention on the instructed stimulus.

In both cases we would expect to find some indication in the animals performance. If the animals splits its attention equally over the two stimuli and ignores the instructive cue, we would observe a negative effect on the animals performance in all the trials. However, if the animal focuses its attention on the relevant stimulus only in the later course of the trials, we expect the animal to perform worst predominantly in short trials. Because in these trials the direction change that the animal needs to respond to fell into an early timeframe in which the monkey has not yet focused its attention on the required stimulus, it is less likely that the correct direction change will be identified. 


\section{Acknowledgments}

This work was supported by the German Ministry for Education and Science Grant BMBF 01GQ0433 to the Bernstein Center for Computational Neuroscience, Göttingen and the Volkswagen-Foundation. We thank Dirk Prüße, Leonore Burchardt and Sina Plümer for excellent technical assistance, Janina Huer for the assistance in the experiments and Shubo Chakrabarti for helpful comments on the manuscript. 


\section{References}

Anton-Erxleben K, Stephan VM, Treue S (2009) Attention reshapes centersurround receptive field structure in macaque cortical area MT. Cerebral Cortex 19(10): 2466-2478.

Britten K, Heuer HW (1999) Spatial summation in the receptive fields of MT neurons. Journal of Neuroscience 19(12): 5074-5084.

Chelazzi L, Miller E, Duncan J, Desimone R (2001) Responses of neurons in macaque area V4 during memory-guided visual search. Cerebral Cortex 11(8):761-772.

Chen Y, Martinez-Conde S, Macknik SL, Bereshpolova Y., Swadlow HA, Alonso JM (2008) Task difficulty modulates the activity of specific neuronal populations in primary visual cortex. Nature Neuroscience, 11(8): 974-982.

Connor CE, Preddie DC, Gallant JL, Van Essen DC (1996) Responses in area V4 depend on the spatial relationship between stimulus and attention. Journal of Neuroscience, 17(9):3201-3214.

Connor CE, Preddie DC, Gallant JL, Van Essen DC (1997) Spatial attention effects in macaque area V4. Journal of Neurophysiology, 75:1306-1309.

Croner LJ, Albright TD (1999) Segmentation by color influences responses of motion-sensitive neurons in the cortical middle temporal visual area. Journal of Neuroscience 19(10):3935-3951.

Ghose GM, Maunsell, JHR (2008) Spatial summation can explain the attentional modulation of neuronal responses to multiple stimuli in area V4. Journal of Neuroscience 28(19): 5115-5126.

Heuer HW, Britten KH (2002) Contrast dependence of response normalization in area MT of the rhesus macaque. Journal of Neurophysiology 88(6):3398-3408.

Katzner S, Busse L, Treue S. (2006) Feature-based attentional integration of color and visual motion. Journal of Vision 6(3): 269-284.

Lankheet MJM, Verstraten FAJ (1995) Attentional modulation of adaptation to two-component transparent motion. Vision Research 35(10): 1401-1412.

Lee J, Maunsell JHR (2009) A normalization model of attentional modulation of single unit responses. PloS One 4(2):e4651.

Lee J, Maunsell JHR (2010) Attentional modulation of MT neurons with single or multiple stimuli in their receptive fields. Journal of Neuroscience 30(8):3058-3066. 
Livingstone MS, Pack CC, Born RT (2001) Two-dimensional substructure of MT receptive fields. Neuron 30:781-793.

Luck S, Chelazzi L, Hillyard S, Desimone R (1997) Neural mechanisms of spatial selective attention in areas V1, V2, and V4 of macaque visual cortex. Journal of Neurophysiology 77(1):24-42.

Majaj N, Carandini M, Movshon JA (2007) Motion integration by neurons in macaque MT is local, not global. Journal of Neuroscience 27(2): 366-370.

Maunsell JH, Treue S (2007) Feature-based attention in visual cortex. Trends in Neuroscience 29(6): 317-322.

Martinez-Trujillo JC, Treue S (2004) Feature-based attention increases the selectivity of population responses in primate visual cortex. Current Biology 14(9):744-751.

McAdams C, Maunsell J (1999) Effects of attention on orientation-tuning functions of single neurons in macaque cortical area V4. Journal of Neuroscience 19(1):431441.

Moran J, Desimone R (1985) Selective attention gates visual processing in the extrastriate cortex. Science 229(4715): 782-784.

O'Craven KM, Rosen BR, Kwong KK, Treisman A, Savoy RL (1997) Voluntary attention modulates fMRI activity in human MT-MST. Neuron 18(4): 591-598.

Patzwahl DR, Treue S (2009) Combining spatial and feature-based attention within the receptive field of MT neurons. Vision Research 49(10): 1188-1193.

Posner MI (1980) Orienting of attention. Quarterly Journal of Experimental Psychology 32(1): 3-25.

Qian N, Andersen RA (1994) Transparent motion perception as detection of unbalanced motion signals II: Physiology. Journal of Neuroscience 14(12):73677380.

Recanzone GH, Wurtz RH (2000) Effects of attention on MT and MST neuronal activity during pursuit initiation. Journal of Neurophysiology 83(2): 777-790.

Recanzone GH, Wurtz RH, Schwarz U. (1997) Responses of MT and MST neurons to one and two moving objects in the receptive field. Journal of Neurophysiology 78(6): 2904-2915.

Reynolds JH, Chelazzi L, Desimone R (1999) Competitive mechanisms subserve attention in macaque areas V2 and V4. Journal of Neuroscience 19(5): 1736-1753. 
Reynolds JH, Chelazzi L (2004) Attentional modulation of visual processing. Annual Review of Neuroscience 27:611-647.

Reynolds JH, Desimone R (2003) Interacting roles of attention and visual salience in V4. Neuron 37(5): 853-863.

Rust NC, Mante V, Simoncelli EP, Movshon JA (2006) How MT cells analyze the motion of visual patterns. Nature Neuroscience 9 (10): 1421-1431.

Salinas E, Abbott L (1997) Invariant visual responses from attentional gain fields. Journal of Neurophysiology 77(6):3267-3272.

Simoncelli EP, Heeger DJ (1998) A model of neural responses in visual area MT. Vision Research 38(5):743-761.

Snowden RJ, Treue S, Erickson RE, Andersen RA (1991) The response of area MT and V1 neurons to transparent motion. Journal of Neuroscience 11(9): 27682785.

Snowden RJ, Verstraten FAJ (1999) Motion transparency: making models of motion perception transparent. Trends in Cognitive Sciences 3(10): 369-377.

Spitzer H, Desimone R, Moran J (1988) Increased attention enhances both behavioral and neuronal performance. Science 240(4850): 338-40.

Spitzer H, Richmond BJ (1991) Task difficulty: ignoring, attending to, and discriminating a visual stimulus yield progressively more activity in inferior temporal neurons. Experimental Brain Research 83(2): 340-348.

Suzuki N, Watanabe O (2009) Perceptual costs for motion transparency evaluated by two performance measures. Vision Research 49:2217-2224.

Treue S (2001) Neural correlates of attention in primate visual cortex. Trends in Neurosciences 24(5):295-300.

Treue S, Hol K, Rauber HJ (2000) Seeing multiple directions of motion Physiology and psychophysics. Nature Neuroscience 3(3):270-276.

Treue S, Martinez-Trujillo JC (1999) Feature-based attention influences motion processing gain in macaque visual cortex. Nature 399(6736):575-579.

Treue S, Maunsell JHR (1996) Attentional modulation of visual motion processing in cortical areas MT and MST. Nature 382(6591):539-541.

Turatto M, Vescovi M, Valsecchi M (2007) Attention makes moving objects be perceived to move faster. Vision Research 47: 166-178.

Wannig A, Rodríguez V, Freiwald Wa (2007) Attention to surfaces modulates motion processing in extrastriate area MT. Neuron 54(4):639-651. 
Watanabe O, Kikuchi M. (2005) Nonlinearity of the population activity to transparent motion. Neural Networks 18(1):15-22.

Womelsdorf T, Anton-Erxleben K, Pieper F, Treue S. (2006) Dynamic shifts of visual receptive fields in cortical area MT by spatial attention. Nature Neuroscience 9(9):1156-1160.

Womelsdorf T, Anton-Erxleben K, Treue S. (2008) Receptive field shift and shrinkage in macaque middle temporal area through attentional gain modulation. Journal of Neuroscience 28:8934-8944.

Yantis S, Serences JT (2003) Cortical mechanisms of space-based and objectbased attentional control. Current Opinion in Neurobiology 13(2):187-193.

Zemel RS, Pillow J (2000) Encoding multiple orientations in recurrent networks. Neurocomputing 32-33:609-616. 


\section{Figure 1}

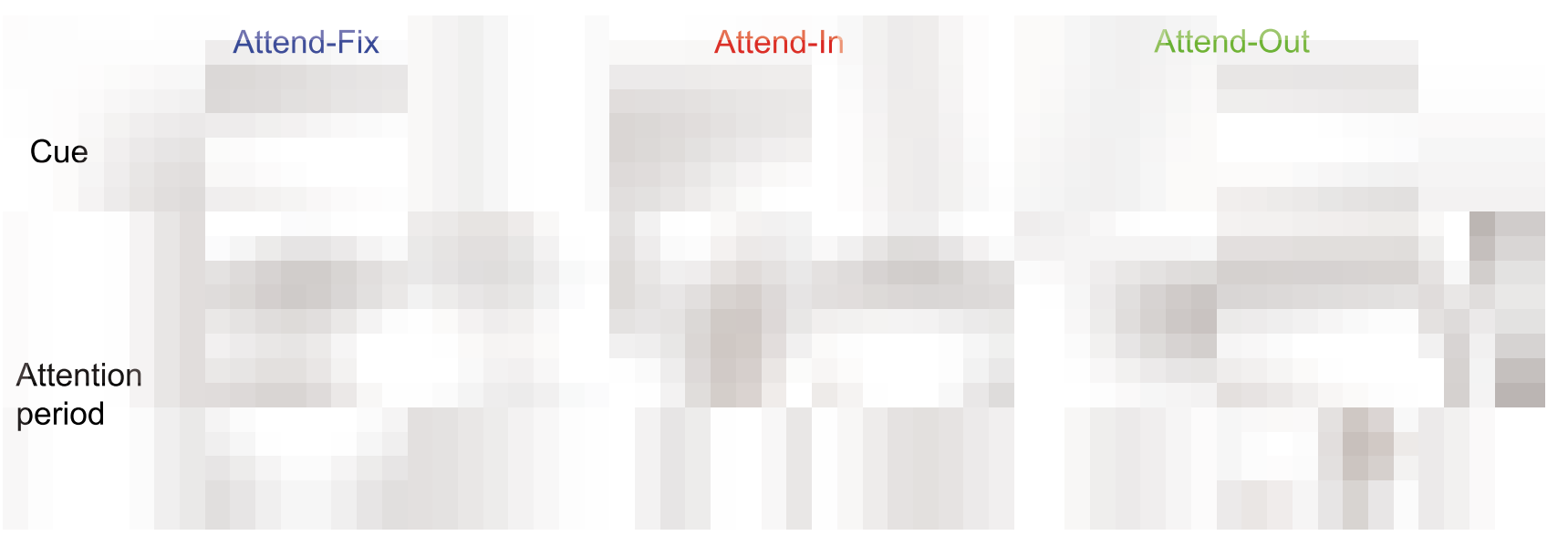

Stephan, Lochte, Kozyrev, Veith \& Treue, Attention on transparent motion

Fig. 1 Schematic trial structure. Each trial began when the monkey foveated a central point. While fixation was maintained, a cue was presented, indicating the location and the direction that the monkey had to attend to after a subsequent interstimulus interval (ISI). In the „attend-in“ condition, the cue indicates a position inside the receptive field (dashed ellipse); vice versa in the "attend-out" condition. Transparent random dot motion patterns (directional separation was kept at an angle of $120^{\circ}$; neuronal response were sampled at every $30^{\circ}$ ) of the same directional configuration were then shown inside the receptive field and in the opposite hemifield. The monkey had to respond to an acceleration in the cued direction and ignore potential accelerations in any of the three remaining distractor directions. The red arrow indicates the target direction, the yellow arrows represent the distractor directions, the thick red arrow indicate the response event (speed acceleration) in the target direction. In the sensory condition („attend-fix“), no cue was presented and the animal had to ignore the transparent stimuli and had to respond to a luminance change of the fixation point. 


\section{Figure 2}

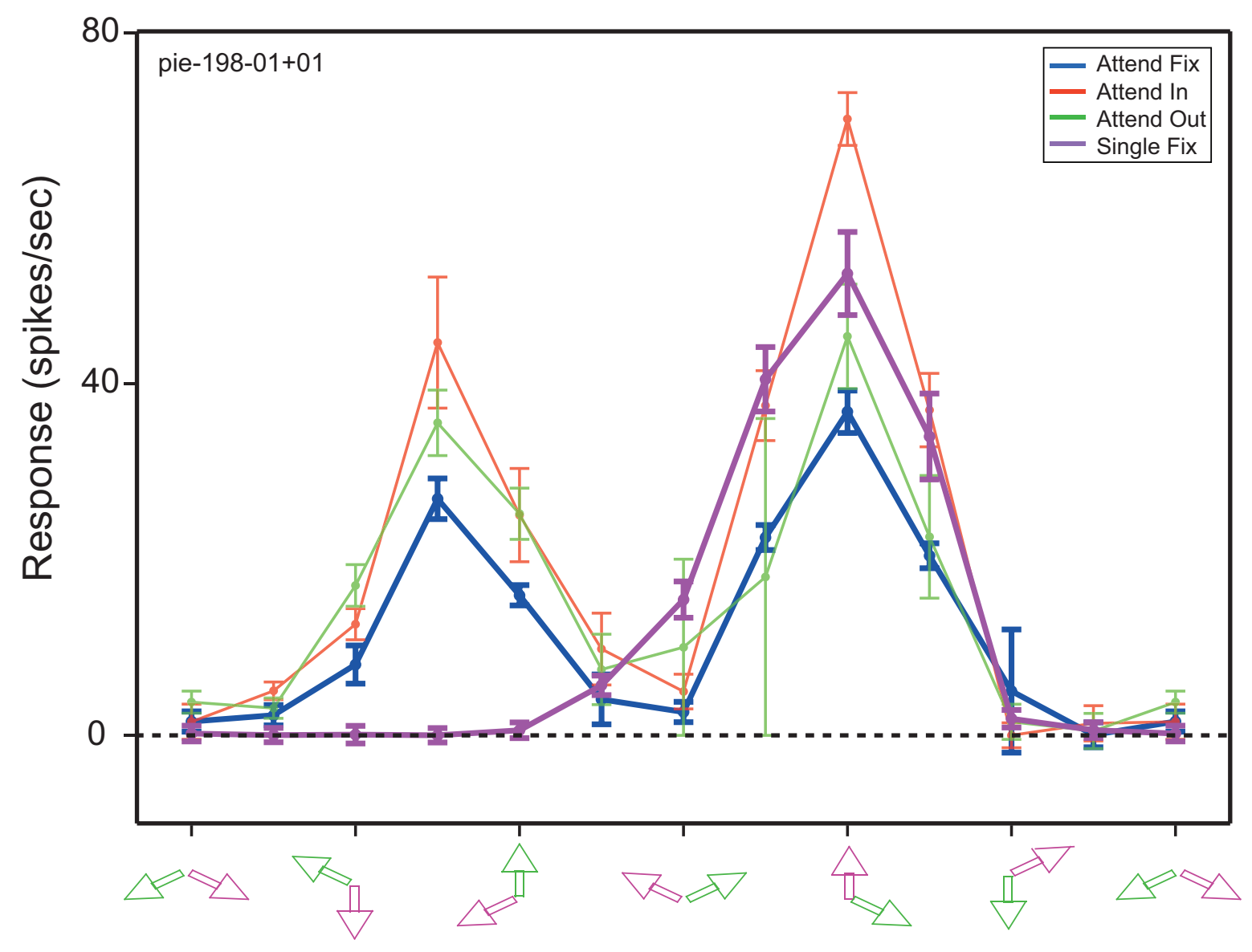

Stephan, Lochte, Kozyrev, Veith \& Treue, Attention on transparent motion

Fig. 2 Tuning curves of an example cell (pie-198-01+01). The average firing rate of an MT neuron (ordinate) to a transparent random dot motion pattern in the attend-in (red), attend-out (green) and the attend-fix (blue) condition is plotted as a function of the direction combinations presented (abscissa). An upward pointing arrow represents the preferred direction, a magenta arrow represents the attended direction (which was always offset by $120^{\circ}$ counter-clockwise from the other direction). Error bars represent standard errors of the meand firing rate. Three curves show the presence of two peaks corresponding to configurations when one of the two patterns moved in the neuron's preferred direction (vertical black dashed lines). In contrast, the single-fix curve depicts the response of the neuron, when only a single direction is presented within its receptive field (see text for details). Consequently, the curve shows only a single peak according to the neurons preferred direction. 


\section{Figure 3}

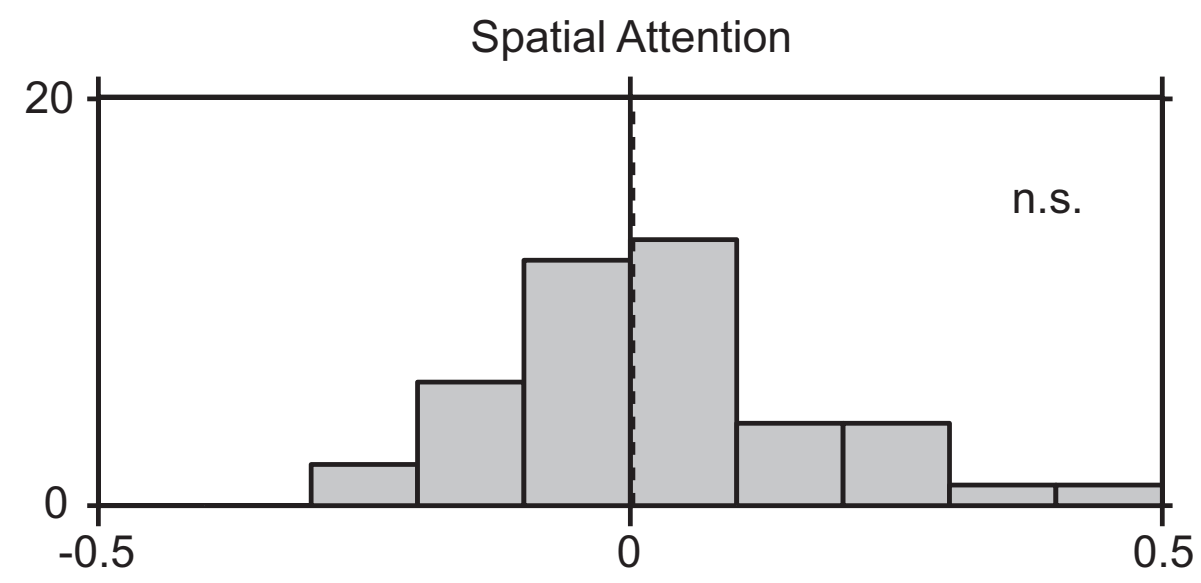

Feature-based Attention

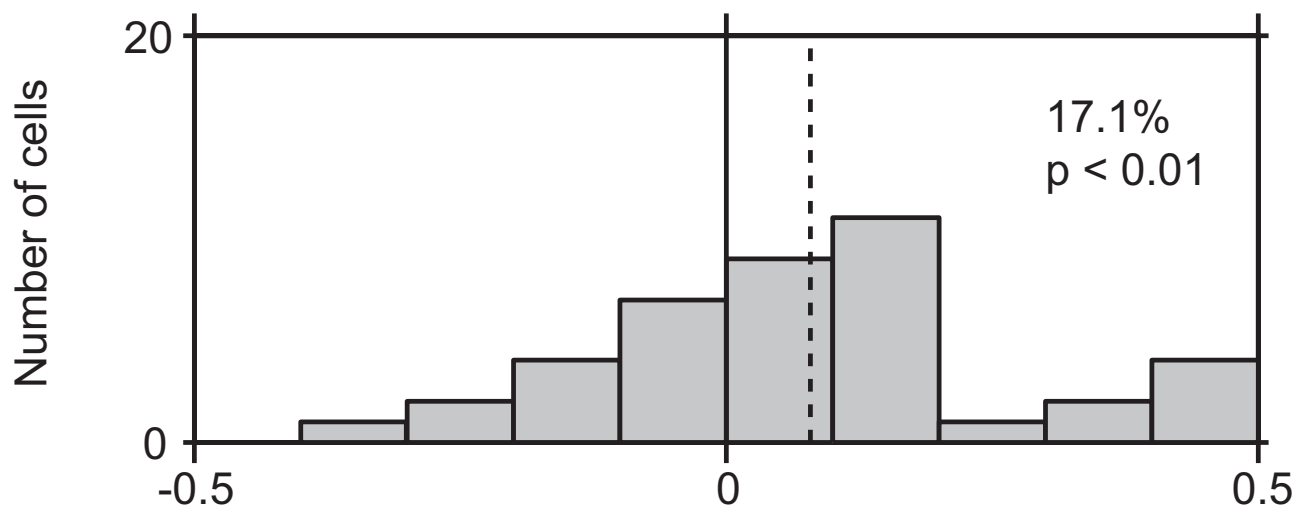

Stephan, Lochte, Kozyrev, Veith \& Treue, Attention on transparent motion

Fig. 3 Attentional modulation by spatial and feature-based attention

The histogram shows the distribution of spatial and feature-based attentional modulation of the response to the preferred direction across the population of 44 cells. Binning of the $\mathrm{x}$-axis is according to the attentional index. The vertical black dashed line marks the mean of the indices. The corresponding average modulation ratio $(17 \%$ for feature-based attention, 'not significant' for spatial attention) is provided in the insertion to the histogram. 


\section{Figure 4}

a)

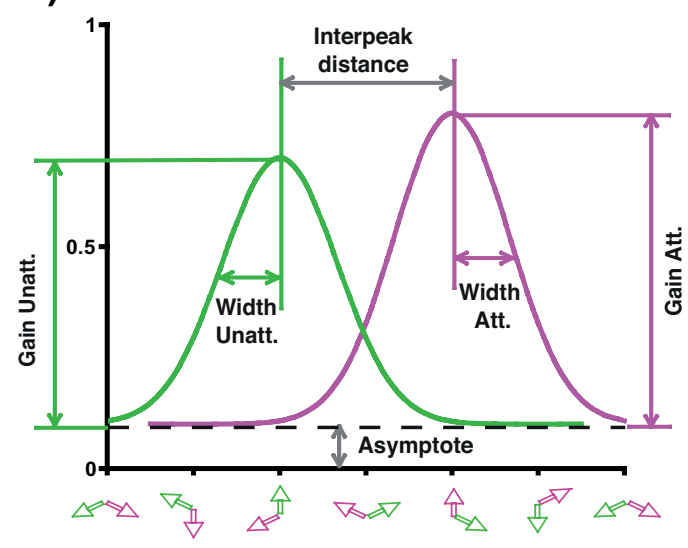

b)

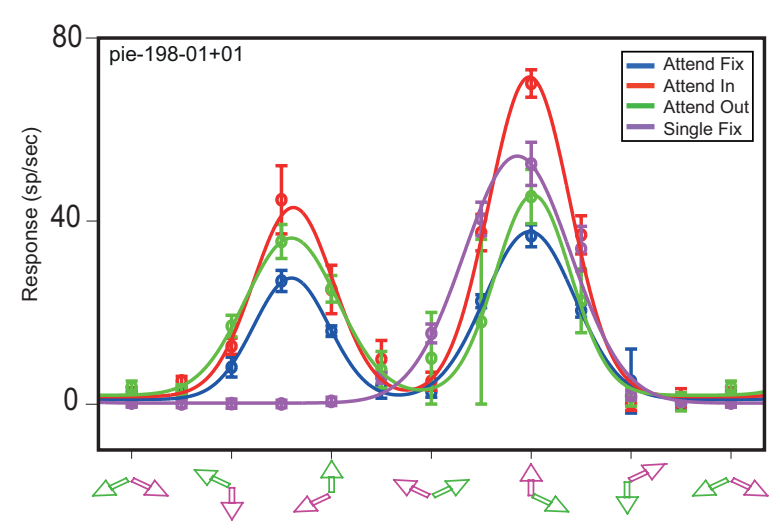

Stephan, Lochte, Kozyrev, Veith \& Treue, Attention on transparent motion

Fig. 4 (a) Schematic overview of the parameters used for the data fitting to the sum of two Gaussians. The attended component is shown in magenta, the unattended component in green.

(b) Example fit of tuning curves of cell pie-198-01+01 by the sum of two Gaussians. Layout is similar to the upper plot of fig. 2a. The firing rates in the attend-fix (blue), attend-in (red), attend-out (green) and single-fix (purple) conditions are shown together with error bars (1s.e.m.). The sum of Gaussians fits are shown by solid lines of the respective color. 
Figure 5

A ) fix/out (spatial \& feature-based attention)

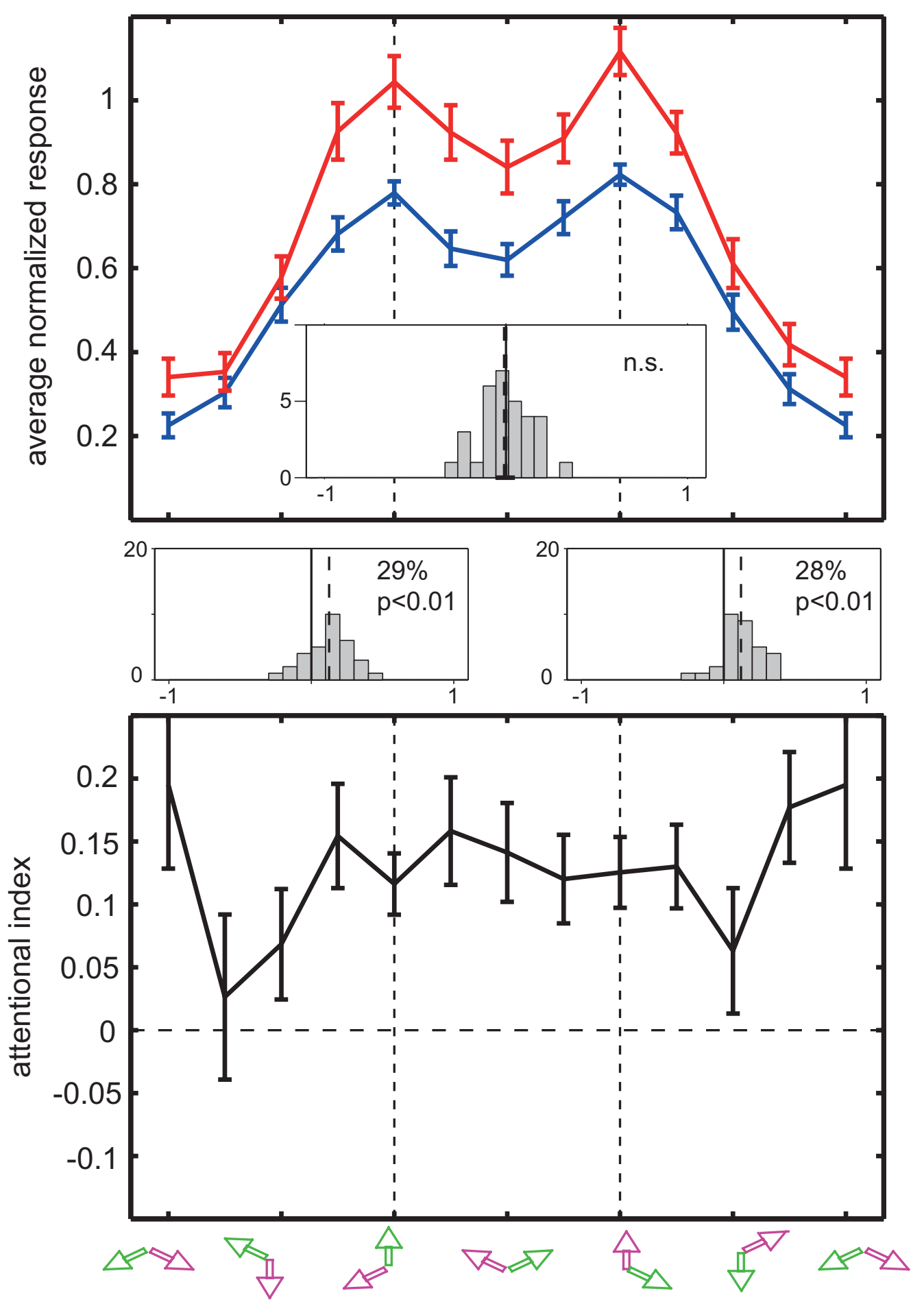


B) in/out (spatial attention)

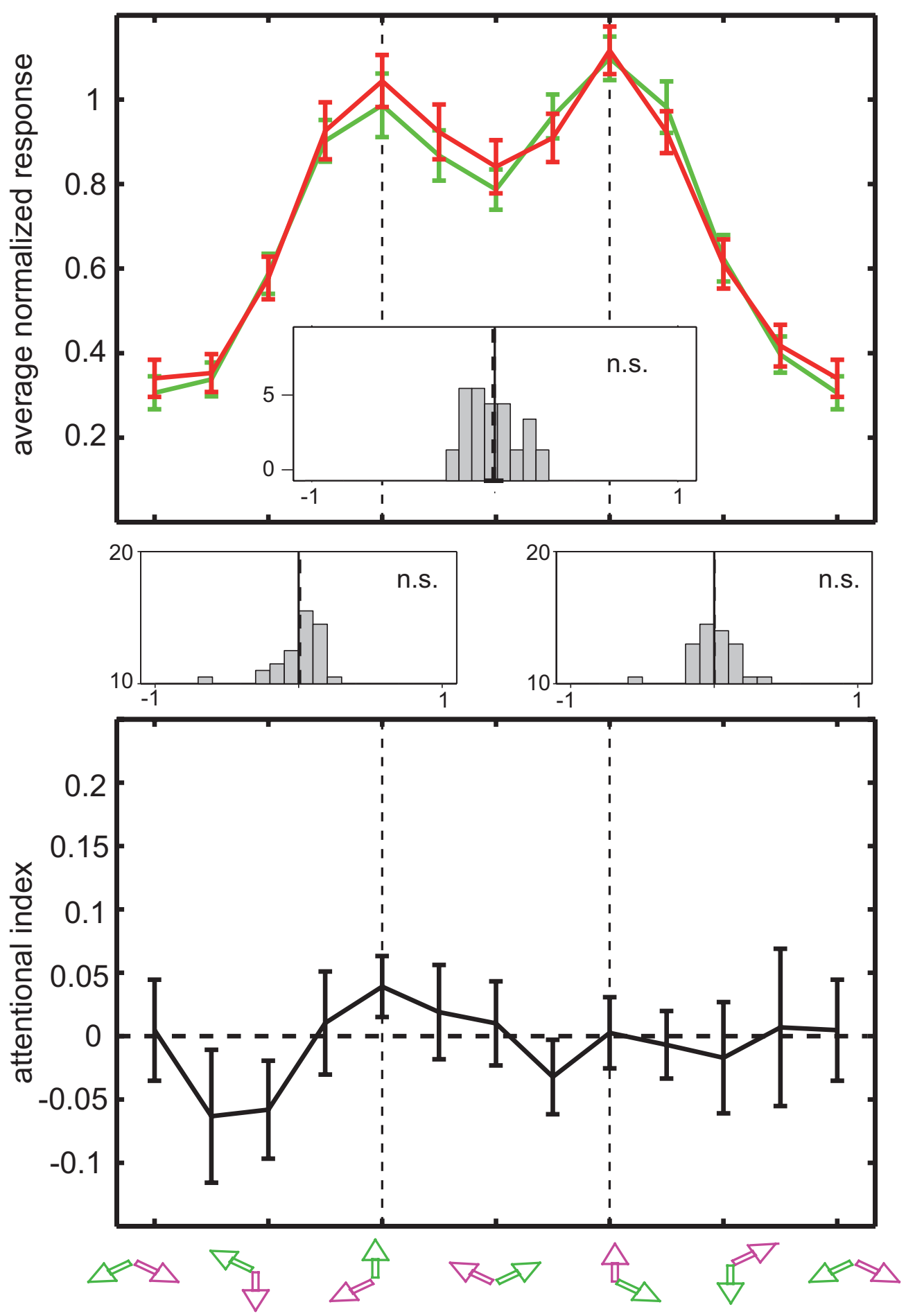


C) fix/out (feature-based attention)

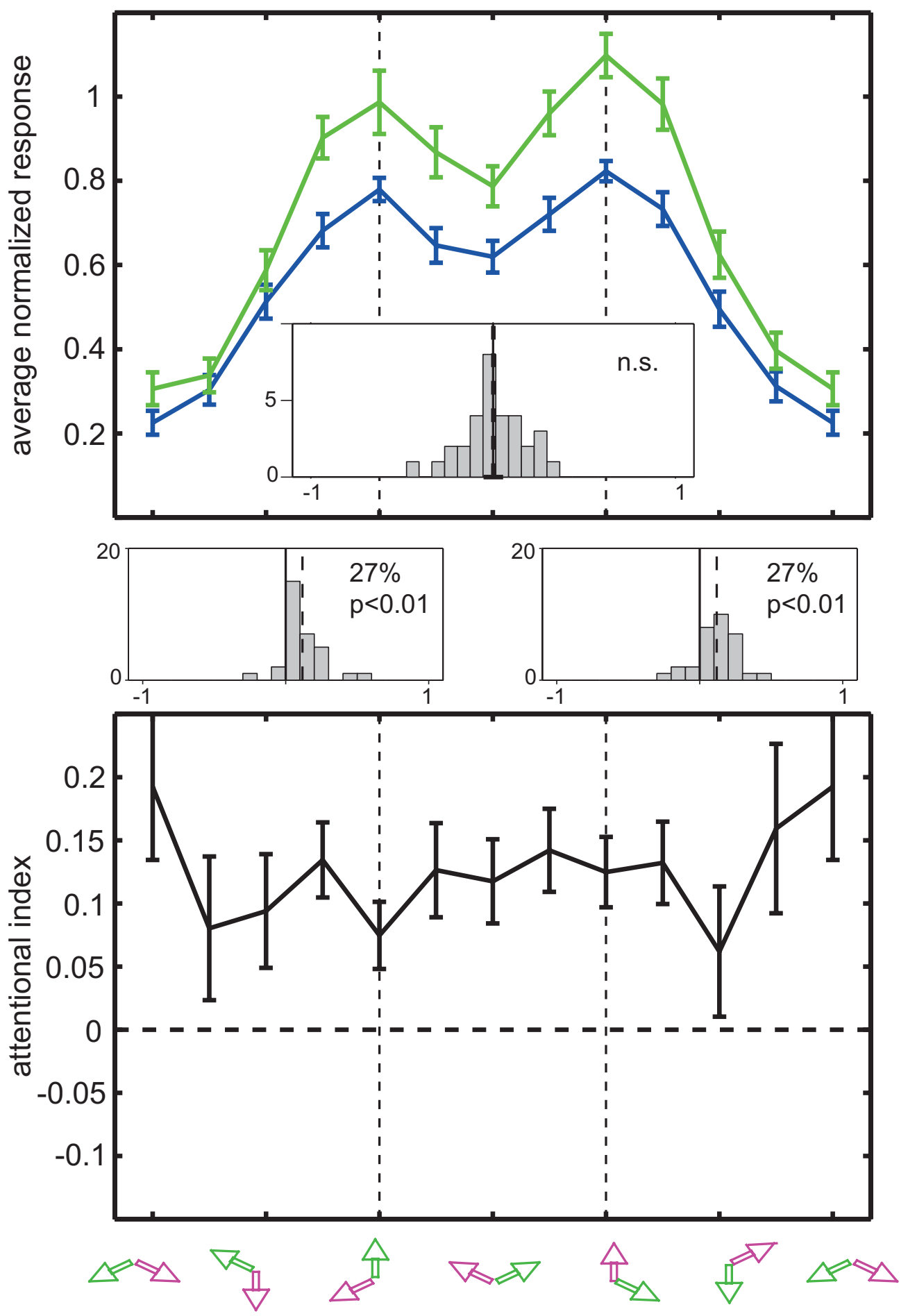

Stephan, Lochte, Kozyrev, Veith \& Treue, Attention on transparent motion 
Fig. 5 Population responses of 44 neurons for each pair of attentional conditions (a) in/fix; b) in/out; c) out/fix). Response profiles averaged across all cells after normalizing the firing rates to the maximal response in the fixation condition (top panel). The layout is analogous to Fig. 2, with each neuron's tuning curve aligned to its particular preferred direction represented by the upward pointing arrow. Error bars represent standard errors across the mean values of each cell's response. The histograms in the top panel show the relative across condition indices of the within-condition peak height ratios calculated within individual tuning curves. Histograms in the middle panel depict the distribution of the fitted Gaussian gains in the subpopulation of 32 neurons. The histograms on the right denote the gain of the Gaussian corresponding to the target component, the left ones those of the distractor component. See text for details.

Mean indices are marked by vertical black dashed lines within the histograms. The mean relative modulation and p-values (t-test) are given in black in the upper right insertions to the histograms. The lower panel shows the modulation profile obtained by averaging the individual attentional indices curves across the 58 neurons. Red stars denote significant indices for those direction combinations including a preferred direction component (marked by vertical black dashed lines). 


\section{Figure 6}

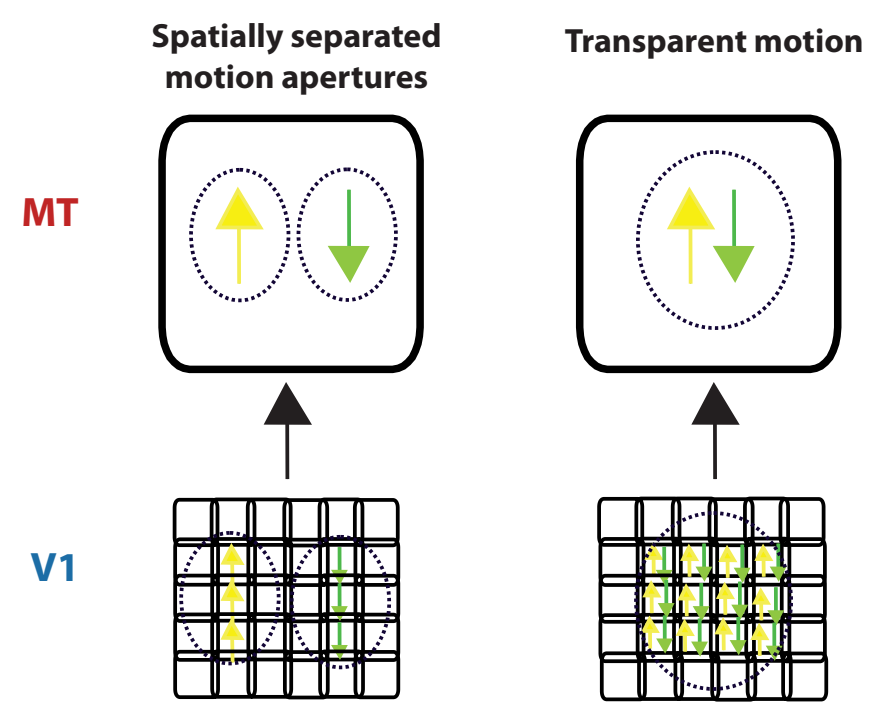

Stephan, Lochte, Kozyrev, Veith \& Treue, Attention on transparent motion

Fig. 6 Schematic overview of the V1 inputs to MT within the tasks with spatially separated and transparent motion stimuli. In the separated stimulus design, different V1 subpopulations feed different motion information into MT, whereas the same neurons in V1 obtain information about both motion components in the transparent motion task design. 
Supplementary Material for

\title{
Attentional modulation of neuronal response in macaque area MT to transparent motion
}

\author{
Valeska M.Stephan, Anja Lochte, Vladislav Kozyrev, Vera Veith \\ and Stefan Treue
}

\section{Supplementary methods}

\section{Fitting procedure}

For a quantitative estimation of modulation in the tuning curves between different attentional conditions, a nonlinear least square fitting method with weights was employed. As weighting factors, inverse values of standard errors at individual data points were used. The standard errors which were smaller than $S E_{\min }=2 / N(n$ 1), where $n$ is the number of repetitions, were corrected to $S E_{\min }$. All calculations were performed with custom scripts written in MATLAB (The MathWorks, Natick, MA) using the Curve Fitting Toolbox.

A periodic Gaussian function was chosen to fit neuronal responses in the unidirectional case (2006):

$$
\begin{aligned}
G_{1}= & a e^{-\frac{\|(x-c)\|_{360}^{2}}{2 b^{2}}}+d \text {, where: } \\
& \|(x-c)\|_{T}=\bmod (x-c+T / 2, T)-T / 2 ; \\
& T=360^{\circ} \text { for the direction-tuned MT neurons. }
\end{aligned}
$$

The 4 parameters of the Gaussian capture the four features of a direction-selective cell: the directional gain or the maximal response modulation (a), the selectivity or tuning width $(b)$, the preferred direction $(c)$ and the response to anti-preferred direction (d) (Treue \& Martinez Trujillo, 1999).

The bidirectional data recorded in the attend-fix, attend-in and the attend-out conditions were fitted using a sum of two periodic Gaussians corresponding to the independent responses to the two stimulus components: 
$G_{2}=a_{1} e^{-\frac{\left\|\left(x-c_{1}\right)\right\|_{360}^{2}}{2 b_{1}^{2}}}+a_{2} e^{-\frac{\left\|\left(x-c_{2}\right)\right\|_{360}^{2}}{2 b_{2}^{2}}}+d$

where indices 1 and 2 denote direction 1 and direction 2 of the RF stimulus respectively. The angular variable $x$ is a mean direction of motion between the two stimulus components; $x$ is counted clockwise from $0^{\circ}=$ upwards. 


\section{Supplementary results}
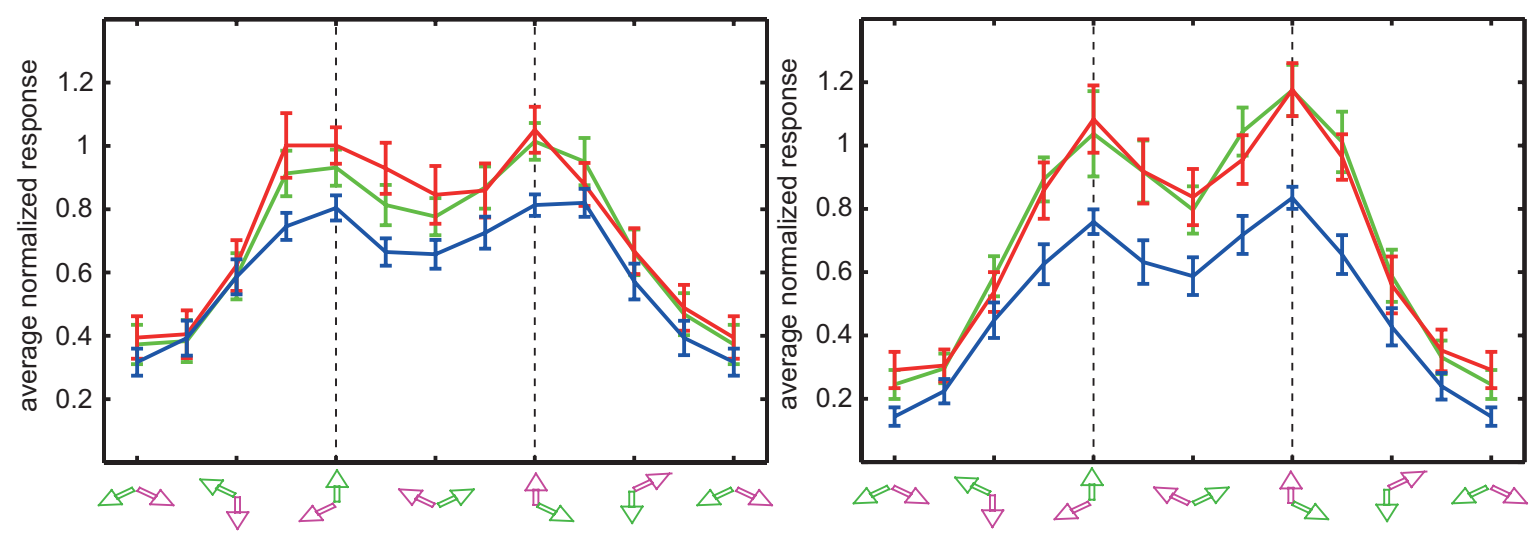

Fig. S1 Activity profiles for three conditions plotted separately for the cell populations of the two monkeys (left: monkey $T=21$, right: monkey $P=22$ ). Layout as in Fig.2. The responses in monkey $\mathrm{P}$ compared to that of monkey $\mathrm{T}$ showed a slightly stronger overall direction-dependent modulation.

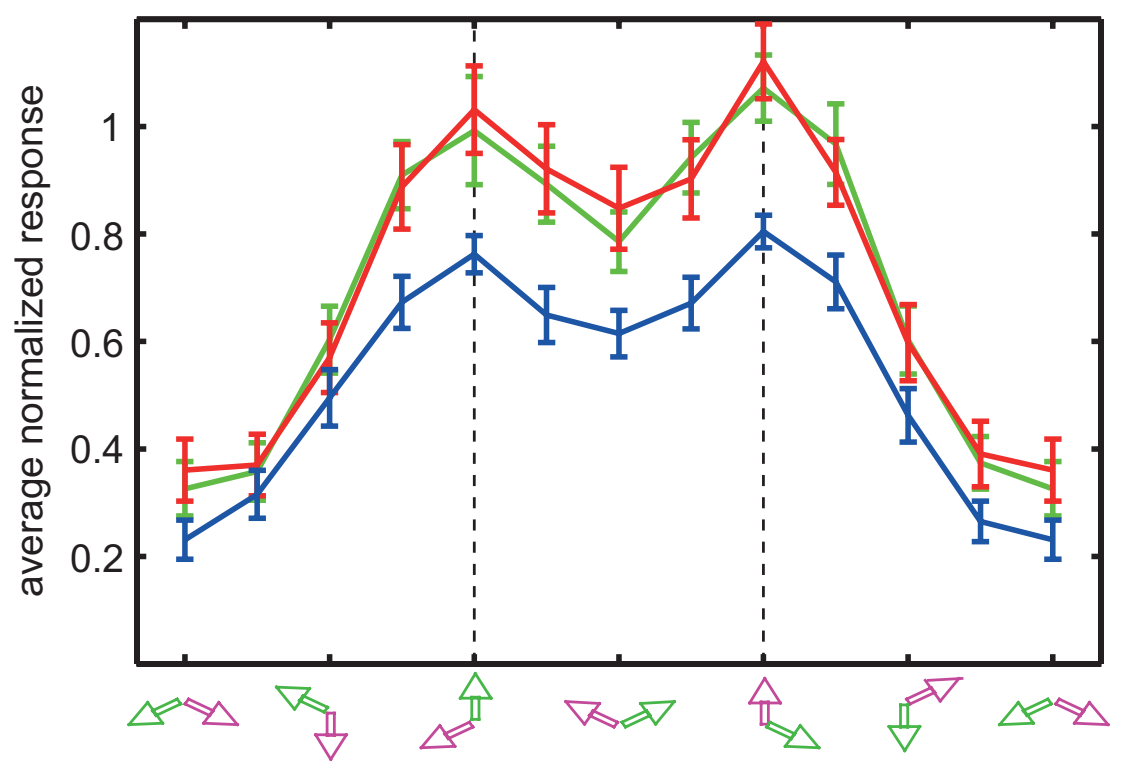

Fig. S2 Activity profiles for the subpopulation of 32 neurons showing significant amplitudes of both Gaussians in the attend-fix condition fit (see Material \& Methods for details). Layout as in Fig.2 and 5. Note that the modulation effects are very similar to those shown for the whole data set in Fig.5 a-c. 

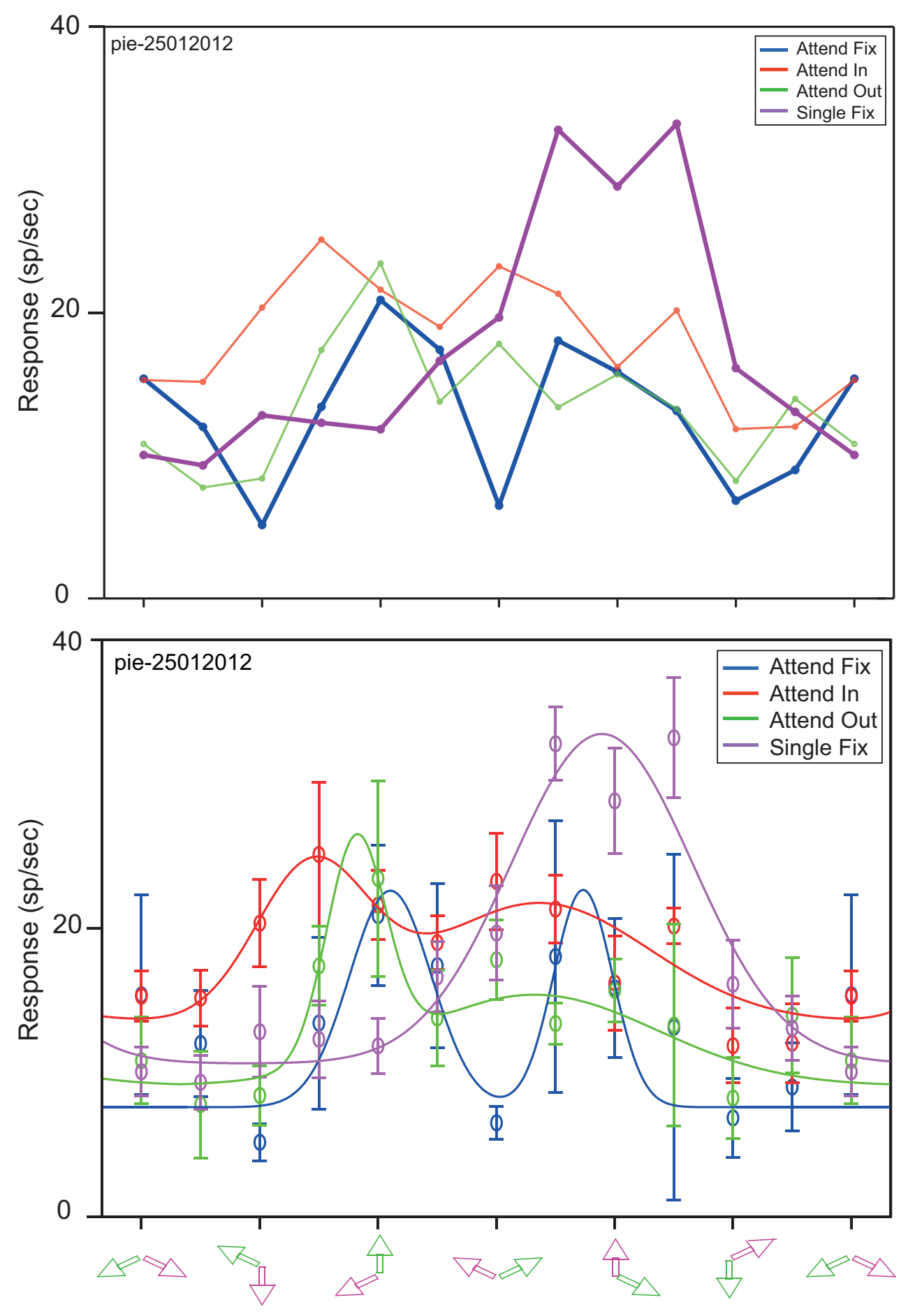

Fig. S3 Tuning curves of an example cell (pie-25012011). The layout is analogue to Fig. 2 and Fig. 4. Errorbars are not shown in the upper panel for better visibility. This cell was not considered for further analysis, as the tuning curve in the attend fix condition (blue curves) did not show a sufficiently bilobed tuning (R-Square < 0.79). The criteria for sufficient tuning is based on the gaussian fittings of the datapoints (lower panel) (see Materials \& Methods for details). 
a)
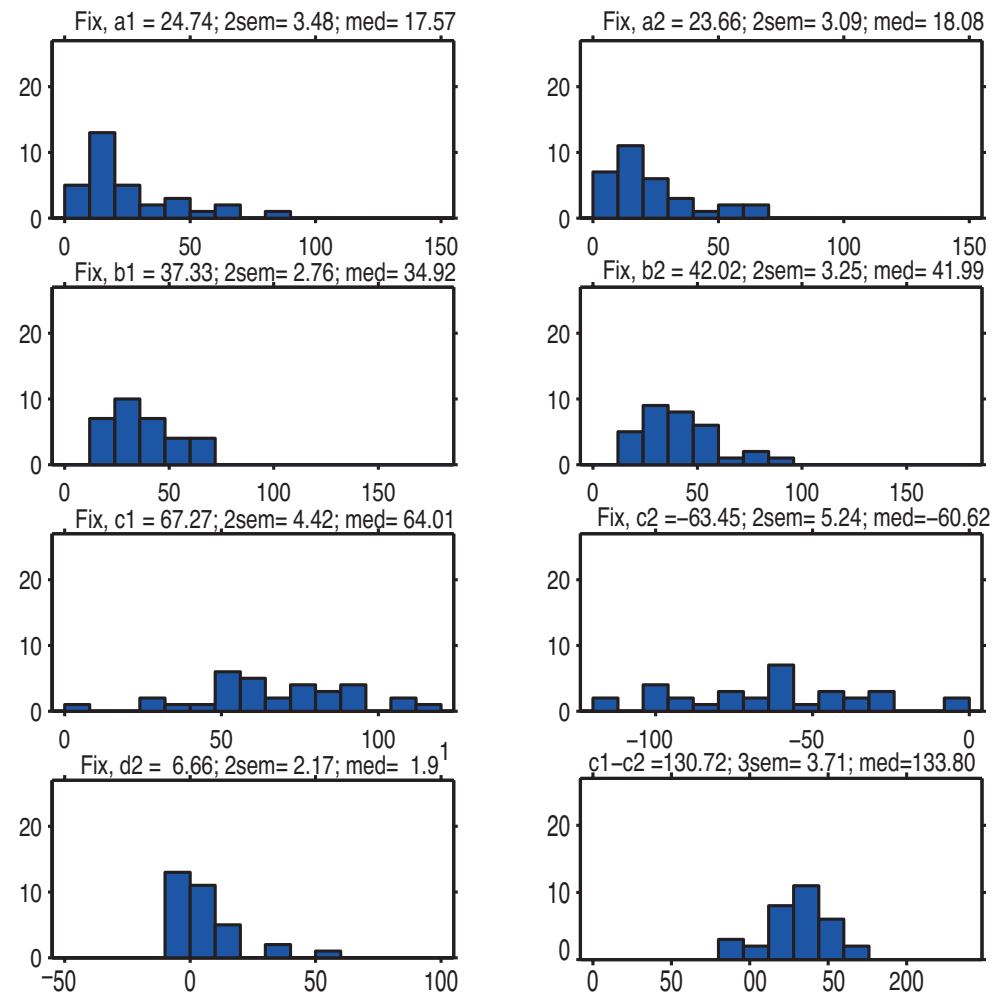

b)
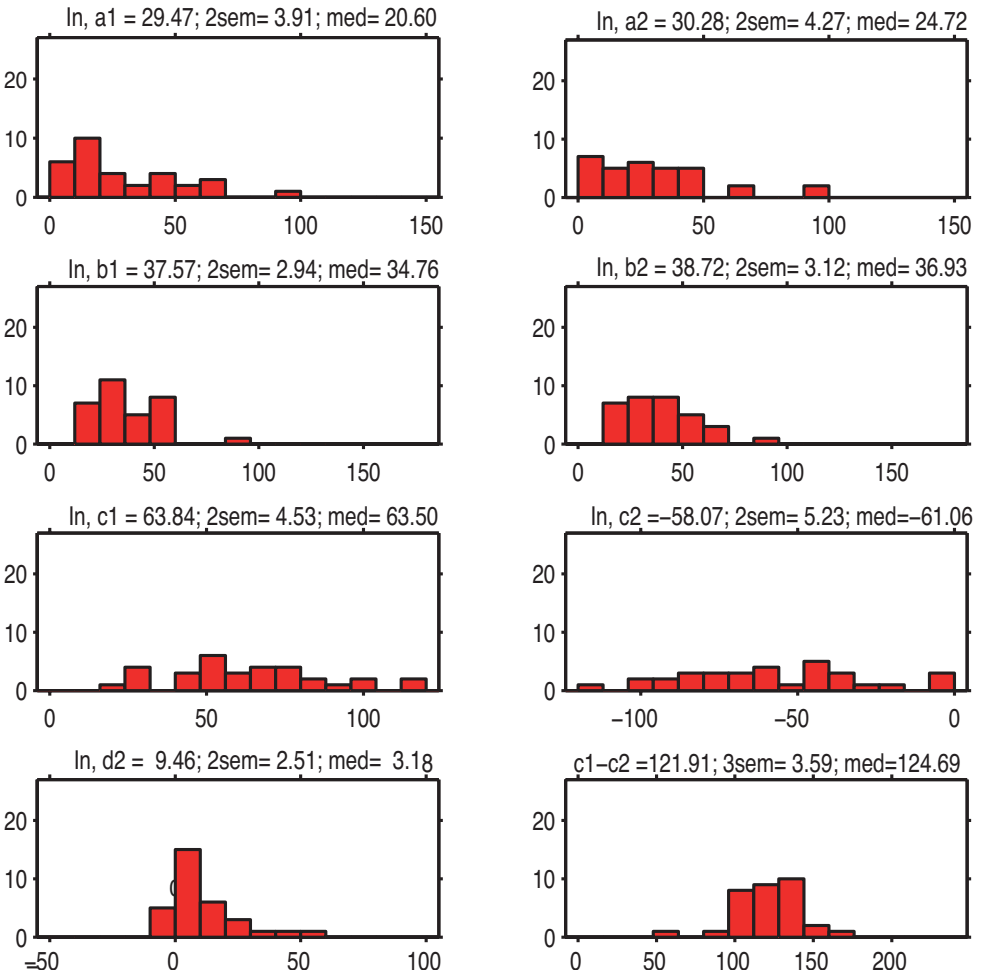
c)
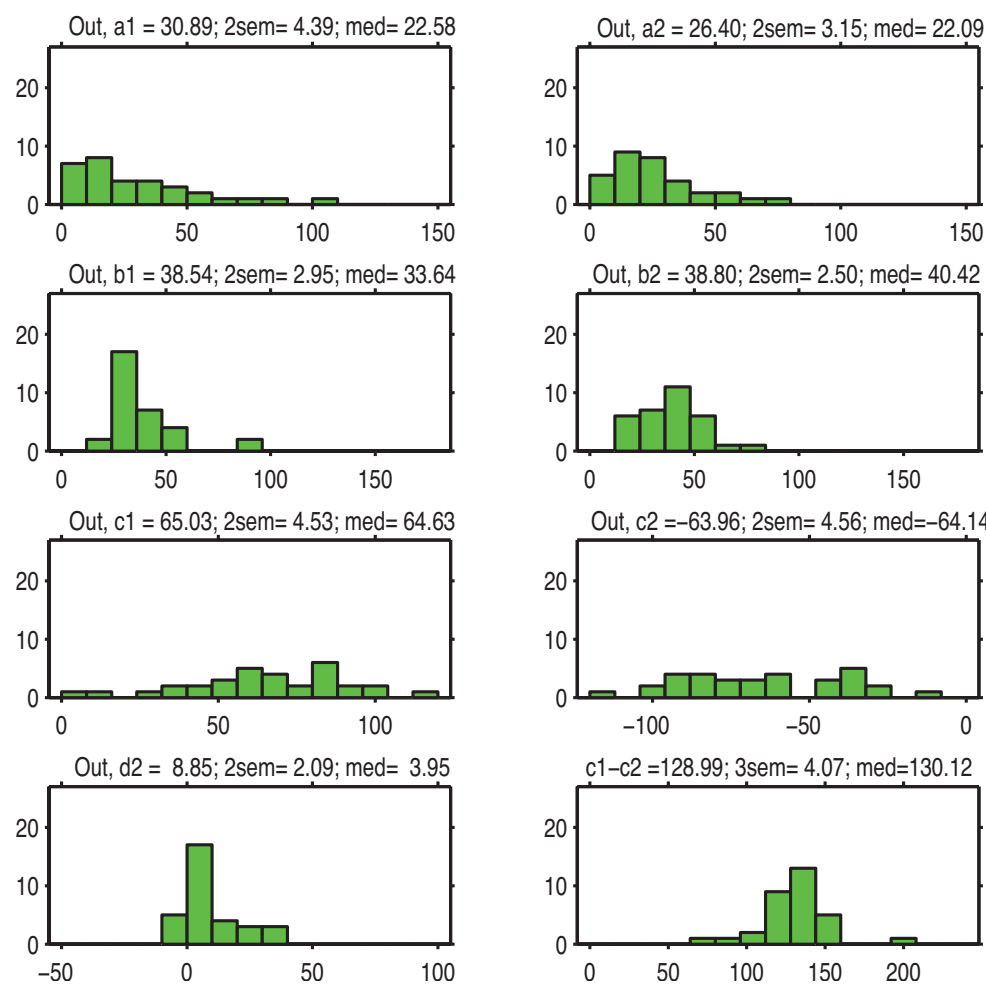

Fig. S4 Distribution of the fitted parameters in the subpopulation of 32 neurons. Xaxes represent the absolute values of the parameters; binning is according to the absolute values of the parameters. Ordinate shows the number of cells in each bin. Parameters of the attend-fix condition fits are depicted in a), those of the attend-in condition in b) and those of the attend-out condition in c). The parameters' values given on top of each histogram correspond to the equation (2). They are presented in form: $<$ parameter $>=<$ mean value $>$; sem $=<$ standard error of mean $>$; med=<median value $>$. c1-c2 (in degrees) is interpeak distance between the two Gaussian components. (see also Fig. 2) 
a) in/fix (feature-spatial attention)
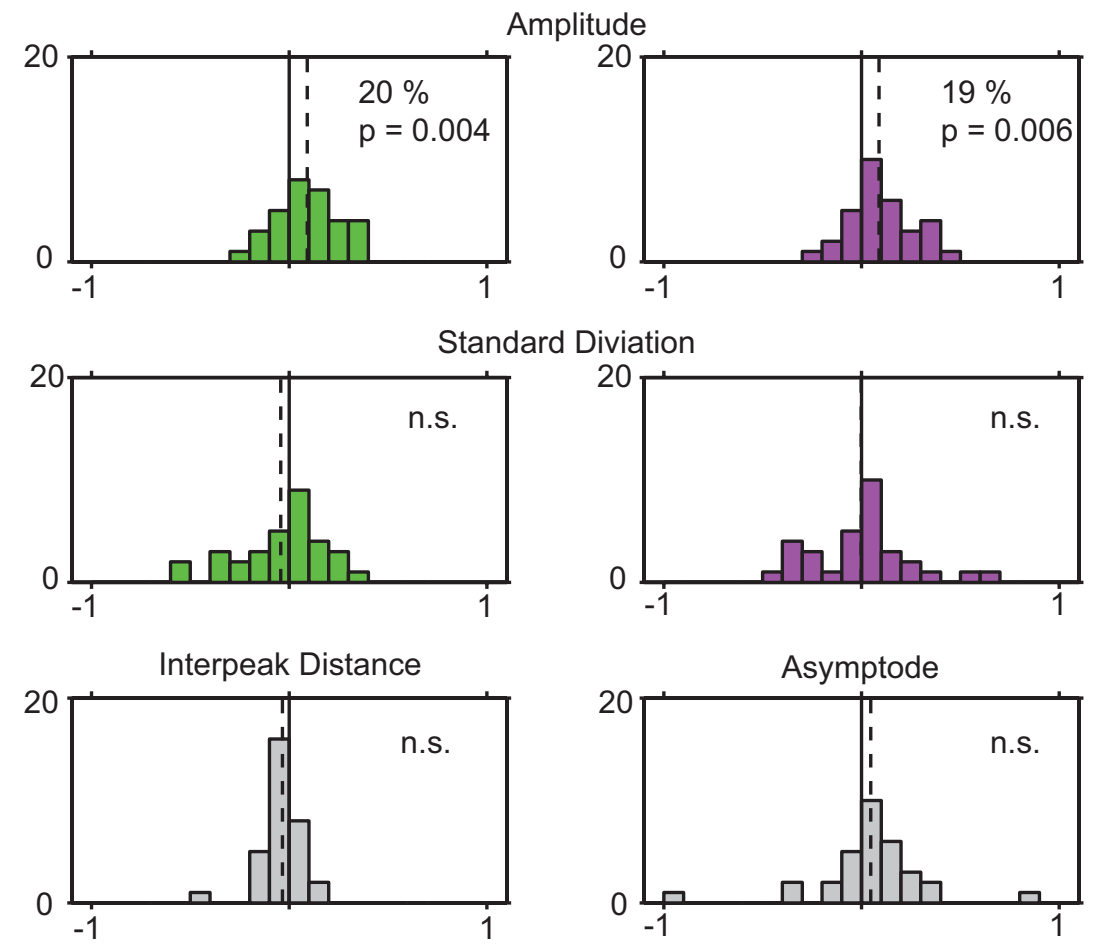

b) in/out (spatial attention)
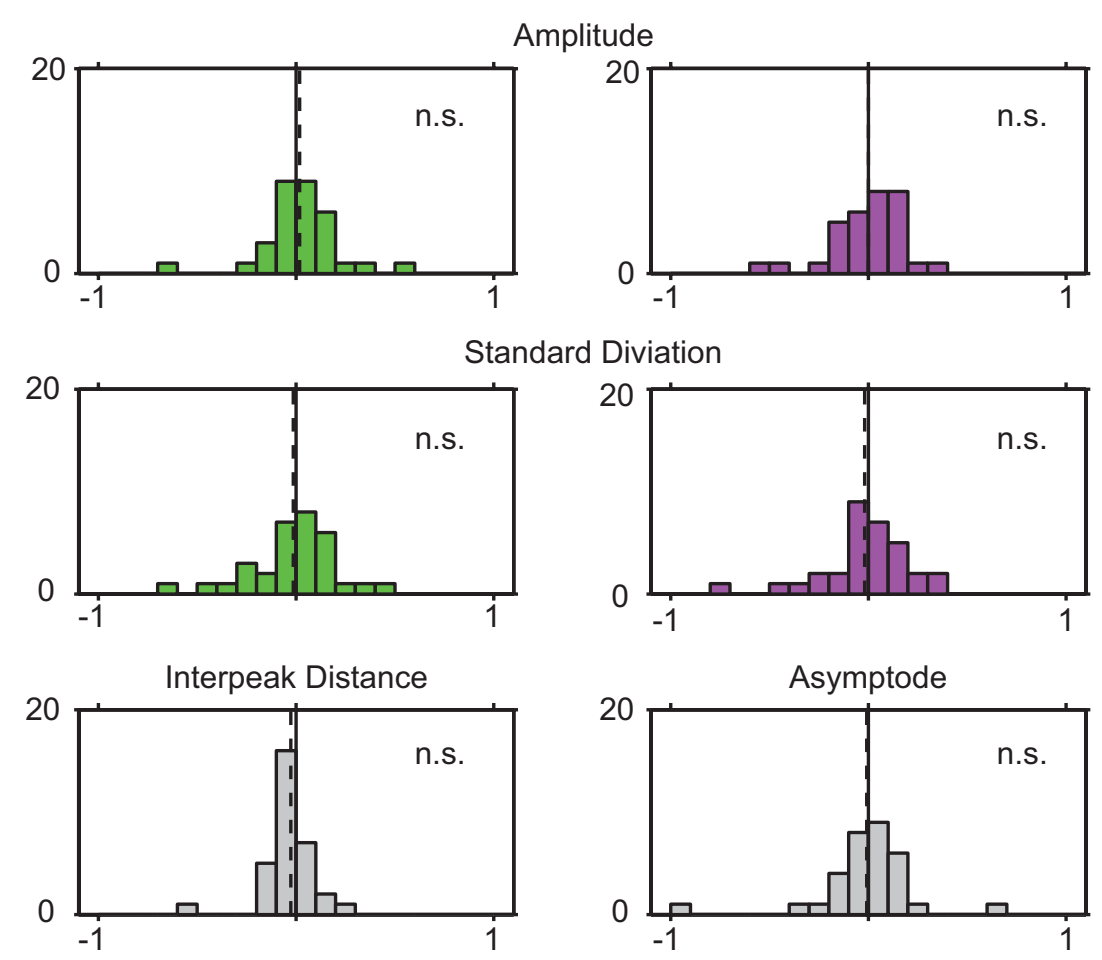
c) fix/out (feature-based attention)
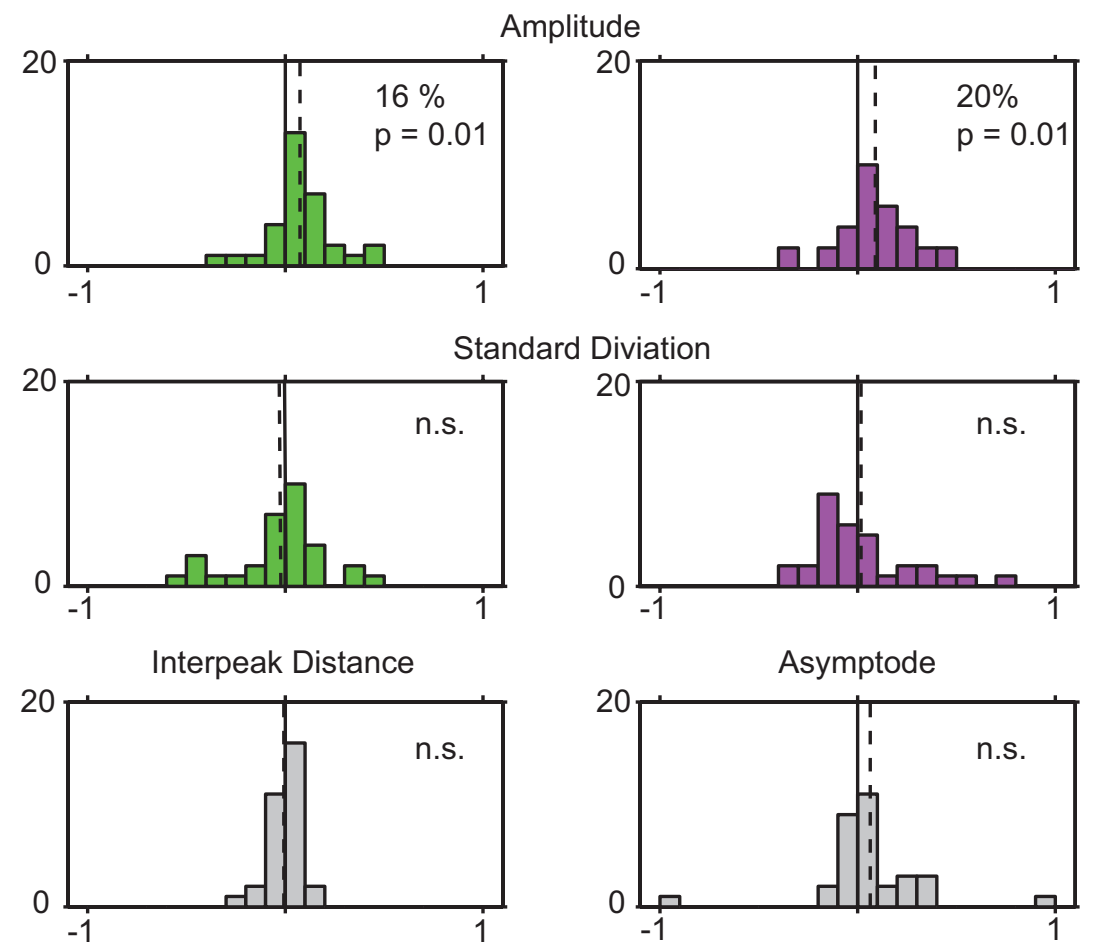

Fig. $\mathbf{S 5}$ shows the distribution of changes of the most relevant fitting parameters (see Materials \& Methods for details) for the three comparisons (a) in/fix; b) in/out; c) out/fix) in the subpopulation of 32 neurons. The ordinate depicts the number of cells per bin. The respective two upper histograms represent attentional changes of the gain of Gaussians corresponding to the attended (magenta) and the unattended (green) patterns. The second row of histograms depicts changes of the components' tuning width for the particular conditions. The lowermost row shows a histogram of the distance between the peaks of the two Gaussians and a histogram of the asymptotic value. Magenta-colored histograms denote the parameters of the component Gaussian corresponding to the target pattern, green-colored ones those of the distractor pattern, gray histograms are relevant to both Gaussian components. Mean indices are marked by vertical black dashed lines. The mean relative modulation and p-values (t-test) are given in black in the upper right insertions to the histograms. „n.s." depicts non significant modulations. Note that, in correspondence to the other results, we only find a modulation of the parameter depicting the gain change in feature-based attention (c) and in the feature/spatial attentional combination (a). 



\subsection{Attentional modulation in V1}

When two stimuli are placed within the receptive field of one neuron in area MT, one can observe a change in the neurons spatial selectivity. The receptive field of the neuron shifts towards the spatial focus of attention. The effect is well described yet the origin is unknown. Two hypothesis might take account for it. One hypothesis states that attentional effects might be inherited' from lower area neurons such as area V1. Alternatively, it might be the case that the attentional effect is not inherited from lower area neurons and rather finds its origin in the synaptic inputs to area MT. We tested which of the two hypothesis can account for the effect on spatial selectivity by recording in area V1 while the monkey did a spatial attention task. We could find that our data does not support the first hypothesis. We concluded therefore that attentional effects are not inherited from lower area neurons to higher area neurons 


\title{
Spatial attentional modulation of
} multi-unit activity in macaque $\mathrm{V} 1$

\author{
Valeska M. Stephan ${ }^{1,2}$, Rui-Feng Lui ${ }^{1}$, Stefan Treue ${ }^{1,2}$ \\ ${ }^{1}$ Cognitive Neuroscience Laboratory, German Primate Center, Kellnerweg 4, 37077 Göttingen, Germany \\ ${ }^{2}$ Bernstein Center for Computational Neuroscience, Bunsenstrasse 10, 37073 Göttingen, Germany
}

\begin{abstract}
Attention is an important mechanism in the visual system to filter out relevant from irrelevant information. Effects of attention have been described for almost every area in the visual processing stream, using different approaches (see e.g. Maunsell \& Cook, 2002; Treue, 2003). In the following study we are interested in the effect of spatial attention on receptive fields in area MT. It was found that shifting ones attention to either one of two visual stimuli within the receptive field of an MT neuron leads to a shift of the receptive field towards that focus of attention (Womelsdorf et al., 2006). Two different mechanisms of how this receptive field shift comes to pass have been proposed. McAdams \& Maunsell (1999) suggested that the receptive field shift is 'inherited' from neurons in lower visual areas, such as V1. According to this hypothesis, spatial attention leads to a multiplicative effect in lower area neurons. This effect is projected to higher area neurons, where it leads to a shift in the receptive field. An alternative hypothesis has been proposed by Womelsdorf et al. (2008). They could find that the shift of the receptive field is best described by a model, which implies a multiplicative effect on neurons in area MT. But, in addition to Maunsell \& McAdams hypothesis, they suggested the alternative hypothesis, that the multiplicative input does not originates in $\mathrm{V} 1$, but comes to pass through a multiplicative weighing on the synaptic inputs into area MT. Unfortunately their data did not provide sufficient information to argue which of these two hypothesis holds true. In this project we want to address this question, by recording from neurons in $\mathrm{V} 1$, while the monkey is doing a spatial attentional task. A spatial attentional effect, which additionally shows multiplicative characteristics, would support the hypothesis from Maunsell \& McAdams. Whereas the absence of an attentional effect or the absence of a multiplicative attentional effect, would rather support the hypothesis by Womelsdorf.
\end{abstract}




\section{Introduction}

Visual attention is known to play a critical role in the processing of visual information. Effects of attention have been described in a wide range of psychophysical experiments and range from enhanced accuracy and reaction time at attended locations to a increase in contrast visibility (e.g. Posner 1980; Carrasco, 2004; Carrasco, 2011). On the neuronal level, it has been found that attention has a variety of effects (e.g. Maunsell \& Cook, 2002; Bisley, 2011; Carrasco, 2011), most of which can be described by a multiplicative effect in the modulation of a neurons firing rate (Maunsell \& McAdams, 2001, Maunsell \& Treue, 2006). A multiplicative scaling of attentional modulation assumes, that while the sensitivity of a neuron is modulated by attention, the selectivity of that neuron does not change. This means that attention increases the firing rate of a neuron by the same magnitude along the tuning curve, but it does not change the shape of the tuning curve, for example the width (Maunsell \& McAdams, 2001). Nevertheless, effects, which do not act according to a multiplicative scaling are not uncommon. The shift of receptive fields in area MT describes an attentional modulation which changes neurons spatial selectivity (Womelsdorf et al., 2006). Two stimuli are placed within the receptive field of one neuron and depending on which of the two stimuli is attended, the receptive field is shifted towards the attended location. While the effect has been implicated by the biased-competition model of attention (Duncan \& Desimone, 1995), the origin of it has not been found, but two hypothesis of how this effect comes to pass have been proposed.

Womelsdorf and colleagues suggested, that while the receptive field shift is caused by a non-multiplicative effect, the origin of this effect could be found in a multiplicative modulation, which is imposed onto the synaptic inputs of the MT neurons (Fig. 1 lower panel, right column). They designed a general gain model, which assumed that attention modulates the spatial weighting of inputs from lower area neurons into area MT in a multiplicative fashion. They could show that their model was well able to describe the observed receptive field modulations and suggested that the effect is the result of a bell-shaped pattern of multiplicative modulations imposed onto the synaptic inputs of the MT neurons (2008). Alternatively, Maunsell\&McAdams $(2001,1999)$ proposed that the receptive field 
shifts could be inherited from multiplicative attentional effects in lower area neurons, such as V1 (Fig. 1 upper panel, right column). MT neurons get their main input through a feedforward projection from the direction selective cells in V1 (Movshon et al., 1996; Van Essen \& Maunsell, 1983). V1 cells process motion on a local scale (Van Essen \& Gallant, 1994) and show a low direction selectivity as a population (Snowdon et al, 1992). However, Movshon and Newsome (1996) could show that V1 cells which project to MT are highly direction selective. They studied neurons in V1 and MT, which are directly linked in their projections. They could show that the direction selectivity of the projecting $\mathrm{V} 1$ neurons ranges in the same magnitude as that of the MT neurons. This result indicates that much of MTs direction selectivity is inherited from $\mathrm{V} 1$ directly and that there is a strong link between MT and V1.

Maunsell \& McAdams hypothesis is build on the link between MT and V1, implying that not only feature preferences are inherited from lower to higher area neurons, but also attentional modulations are projected in a feedforward manner. Maunsell \& McAdams propose a non-multiplicative effect on the receptive field of higher visual neurons as a consequence of multiplicative scaling of the responses in lower area neurons. Receptive fields of higher area neurons are comprised over a spatial extent of several low area receptive fields. If only one of those lower area neurons response is increased by multiplicative scaling, the response would be projected within the local boundaries of its receptive field. As a consequence it would lead to an uneven summation of responses within the higher area receptive field, which then is observed as a shift of the receptive field (Fig. 1).

Both hypothesis are favour a multiplicative attentional effect being on the bottom of the receptive field shift. But while Maunsell \& McAdams propose it to be an inherited feature within the processing hierarchy, Womelsdorf suggests it to be an effect which might be an internal mechanism within an area. Studies, which have shown, that attentional effects in V1 generally show an additive rather then a multiplicative scaling (Thiele et al., 2009; Boynton, 2011; Chen \& Seidemann, 2012), support the later hypothesis.

To gain a further understanding of the mechanisms, which cause a change in spatial selectivity in higher area neurons, we conducted an experiment, in which we recorded from neurons in the area $\mathrm{V} 1$, while the monkey did a spatial attention 
task. If the first hypothesis holds true, we would expect to find that the spatial attentional effects are best described by a multiplicative scaling. However, if we do not find a multiplicative effect, it suggests that this hypothesis does not account for the modulation of receptive fields in area MT.

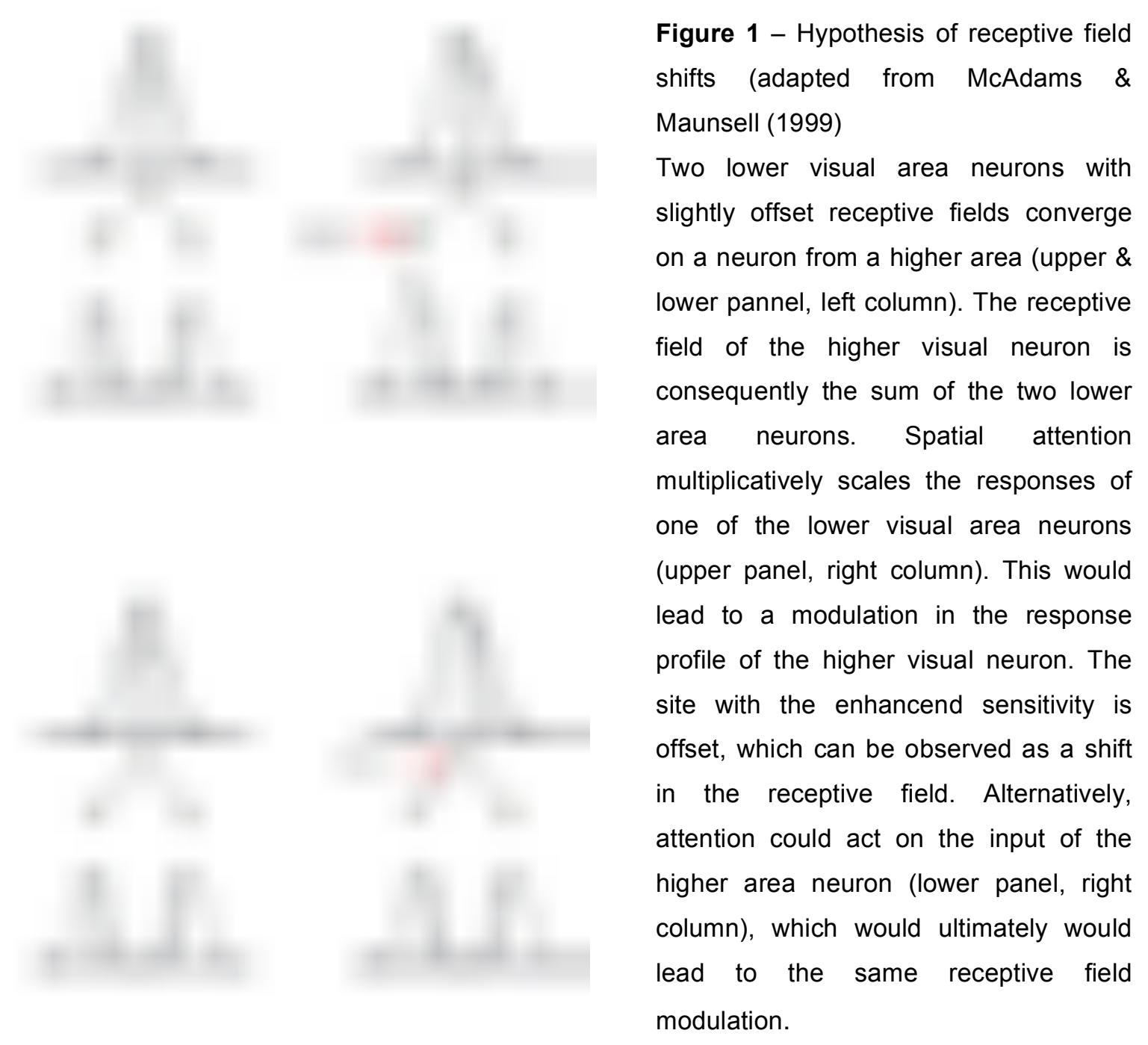

The task was designed to mimic the design of the Womelsdorf (2006) study. Two random dot stimuli are presented to the monkey, both of the same characteristics and placed at the same eccentricity. One stimulus is placed within the receptive field of a V1 neuron, one placed outside the receptive field. The monkey was instructed to attend to either one of the stimuli. While the monkey was doing the task, a probe stimulus was briefly presented at different positions in close proximity of the random dot patterns during the length of the trial. The monkey had to ignore this stimulus. One implication of the task design was that it might activate 
strong attentional effects in V1. Attentional modulation in V1 appears to be particular sensible to stimulus arrangements. It could be shown, that attentional effects might be diminshed or even absent, when only one target stimulus is presented (Luck et al., 1997, McAdams \& Maunsell, 1999). If the stimulus however is embedded within a contextual stimulus or presented in the presences of distracting information, strong attentional effects can be observed (e.g. Motter 1993, McAdams \& Reid, 2003). Furthermore, fMRI studies could show that, compared to higher area, attentional modulation in V1 is pronounced when a small region of space is attended (Müller et al., 2003).

We tested for the implication that the specifics of the task design promote attentional modulation in V1, by introducing a control condition, in which the probe stimulus is not present, therefore removing the distracting environment, which pronounces attentional modulation.

We observe small, but significant attentional effects for the probe condition, but only for a non-direction selective subpopulation. We do not find a significant effect for the non-probe condition, independent of direction selectivity. Furthermore we characterise the attentional effect and do not find a multiplicative effect. Though due to technical shortcomings this result is to be viewed with care.

\section{Materials \& Methods}

For this project we recorded extracellular responses of neurons in the primary visual cortex (V1) of one male rhesus monkey (Macaca mulatta), while the monkey performed a visual spatial attention task. The monkey was surgically implanted with a custom-made orthopaedic implant that prevented head movements during recording, and a multielectrode CerePort Utah Array (Black Rock Microsystems,Salt Lake City, USA) in V1. The surgeries were conducted under aseptic conditions with isoflurane anaesthesia. During working days, the monkey was held under a water controlled schedule and obtained the majority of fluid as reward (either water or juice) during the experiment. All procedures and experiments were approved by the animal ethics committee of the regional government office of Braunschweig. 


\section{1 - Electrophysiological Settings}

Extracellular recordings were obtained with a chronically implanted multielectrode CerePort Utah Array. The array is created from platinum. It consists of 96 electrodes, arranged in a $10 \times 10$ square and is approximately $5 \times 5 \mathrm{~mm}$ in size (Fig. S1). The space between neighbouring electrode is 400 micrometre and each of the electrodes has a length of 1.5 millimetres. The impedance is in the range of 100-800kOhm. Neuronal data was collected with the Omniplex System (Plexon Inc., Dallas, TX). The omniplex system digitizes the signal at $40 \mathrm{kHz} / 16$ bits. The analog low-cut filter was set to 0.5 , the high-cut to $8 \mathrm{kHz}$. The gain amplification of neuronal signals can be between 250-8000.

The selection of the implantation site was based on anatomical MRI scans. A craniotomy was performed and a flap of the dura mater was lifted. Simultaneously, a connector was implanted at the opposite hemisphere to the recording site.

We recorded 28 datasets over 23 days. One dataset refers to one completed experiment with recordings from all 96 electrodes.

\section{2 - Quality of signal}

We observed a rapid decline in the signal quality over the first weeks after implantation of the array (Fig S2). Whereas shortly after implantation we could isolate single units at up to 30 sites, at the start of the recordings we could isolate maximally one unit reliably. Therefore we did not analyse single unit activity, but focused our analysis on multi-unit activity. Due to limitations in the neuronal signal and the layout of the task, we were not able to test for day-by-day stability of the neuronal data we recorded. To test whether neuronal data stays stable over a prolonged period of several days or longer, one necessity is to test the change in the waveform. As our signal had already declined heavily at the start of the experiment we did not find any clear defined waveforms. Therefore we could not test for long-term stability with the help of waveforms. Alternatively, we could have applied indirect measurements as to whether the data of an electrode shows consistency over several days. One type of indirect measurement would have been to measure the direction tuning of the neuronal responses at each electrode 
on a daily basis. Unfortunately, we did not routinely conduct a complete tuning experiment in our data collection. Another indirect measurement could have been the mapping of the receptive fields on a day by day basis. But as the receptive field structure and position was not in our main focus in these experiments, we did not measure receptive fields in regular intervals.

We considered our recordings to be independent recordings and did not test for interaction between close and far positioned electrodes on a day-by-day basis for each electrode.

Note that in the following we will try to avoid terms, which might suggest that we dealt with data from single cells. For the sake of readability, we will sometimes refer to recorded neuronal data from one electrode as data from one unit.

\section{3 - Thresholding}

Due to the quality of the signal we did not analyse single unit activity, but analysed multi-unit activity (MUA). We applied a thresholding method to the data of each recording site in each dataset (Liu \& Newsome, 2002; Pooresmaeili et al., 2010). Because we were interested in the change of the neuronal response rate depending on the sensory input and attentional condition, we determined the threshold in a baseline condition. External noise of the electrode stays constant (Super et al., 2005) therefore any change observed in the MUA data reflects a change of the sampled neuronal data. In the baseline condition only a fixation point was presented on the screen and the monkey had to detect a luminance change in the fixation point (see "Experimental Protocol and Conditions" for details). For each recording site we averaged the neuronal response rate in a time window of $100-700 \mathrm{~ms}$ after trial onset over all trials in the baseline condition. After setting an arbitrary threshold we counted the neuronal events, which crossed the threshold. We compared the frequency of neuronal events to a fixed neuronal response rate of $10 \mathrm{~Hz}(+/-1)$. If the mean neuronal response rate of the baseline condition matched the fixed neuronal response rate, we would apply this threshold to all trials of the remaining conditions of one data set of one electrode. If the mean neuronal response rate would not match the fixed response rate, we would adjust the threshold to a new value and restart the procedure. This method is only 
one of various methods of preparing MUA data for further analysis (e.g. Super et al., 2005; Pooresmaeili et al., 2010; Cohen \& Newsome, 2009). We chose this method over other approaches, because it was a very quick and straightforward way to prepare our data for further analysis. Liu and collagues (2002) used this method for analysing speed tuning in MT and could show that this approach provided a sufficient method to process their data. Furthermore, Super et al. (2005) compared MUA data, which was revised with a similar arbitrary threshold and MUA data, which was administered with a low-pass filtering method. They did not find that any of these two methods had an advantage over the other. Furthermore they could show that MUA data largely provided similarly clear results as recordings from well isolated single neurons.

The reported mean firing rates for cells in the primary visual cortex is between 1 and up to $9 \mathrm{~Hz}$ (Olshausen \& Field, 2005). We chose a threshold of $10 \mathrm{~Hz}(+/-1)$, which is slightly higher then the reported values, but still in an acceptable range to capture differences in firing rates.

\section{4 - Stimulus Presentation \& Behavioural Control}

Visual stimuli were presented with a custom software program running on an Apple Macintosh G4 Computer. The software also monitored and recorded eye position and behavioural responses of the animal. Neuronal data was recorded with the Omniplex System (Plexon Inc, Dallas, USA). During the experiments the monkey was seated in a primate chair $57 \mathrm{~cm}$ in front of a monitor (CRT monitor, LaCie, Electron22 Blue IV; 40 pixels per degree, $75 \mathrm{~Hz}$ refreshrate). Motion stimuli consisted of moving random dot patterns (RDPs) presented within a stationary circular aperture. Dots moving outside the aperture were replaced by dots at random positions at the opposite side of the RDP aperture. RDPs contained 15 dots per square degree of visual angle with individual dots subtending 2 by 2 pixels. Dots were always white $\left(75 \mathrm{~cd} / \mathrm{m}^{2}\right)$ presented on a grey background (24 $\mathrm{cd} / \mathrm{m}^{2}$ ). 


\section{5 - Experimental Protocol and Conditions}

\subsection{1 - Mapping Task}

While the anatomical MRI scans gave us a basic understanding about the position of the array in the cortex, it was still necessary to determine functionally the precise position of the array in the cortex. We conducted several visual field mappings to determine the exact size and positions of the receptive fields for the neuronal signals at each electrode. By comparing this data with data from the literature, we determined the position of our array in the cortex.

After coarsely locating the receptive fields with a manual mapping experiment, to the lower right quadrant of the visual field close to the fovea, we applied a systematic mapping experiment to identify the exact position and size of the receptive fields for every electrode. A fixation point would appear in the middle of the screen, which the monkey had to foveate. By releasing the lever, the monkey had to indicate a small luminance change in the fixation point within a response time window of up to $500 \mathrm{~ms}$ after the response event. If the monkey answered outside the response time window, the trial was not rewarded. Throughout the trial, a $0.4 \times 0.4$ degree white square flashed up for $180 \mathrm{~ms}$ at several regularly interspaced positions on the screen (Fig. 2). The stimulus was presented in a random sequence at approx. 170 positions on a regularly spaced 4.5 by 4.5 degree grid. The grid was centred on the estimated position of the receptive fields. To ensure that the complete area would be mapped by our stimulus we partly overlapped the stimulus positions. The eyeposition of the left eye of the monkey was closely monitored during the experiment. The fixation window was a circle of 1 degree radius. The trial would be aborted if the monkey moved his eyes outside the fixation window. We only included the neuronal response from successfully accomplished trials in our analysis. For every electrode, we determined the mean neuronal response in a time window of $40-140 \mathrm{~ms}$ as a function of the spatial positions of the stimulus. We determined the positions of the maximum response, which we defined as centroid of the receptive field and defined the outline of the receptive field to be the half maximum response (Fig. 3). To verify the position of the array in the cortex, we analysed the position and the size of the receptive fields for every electrode (see Supp. Materials). 

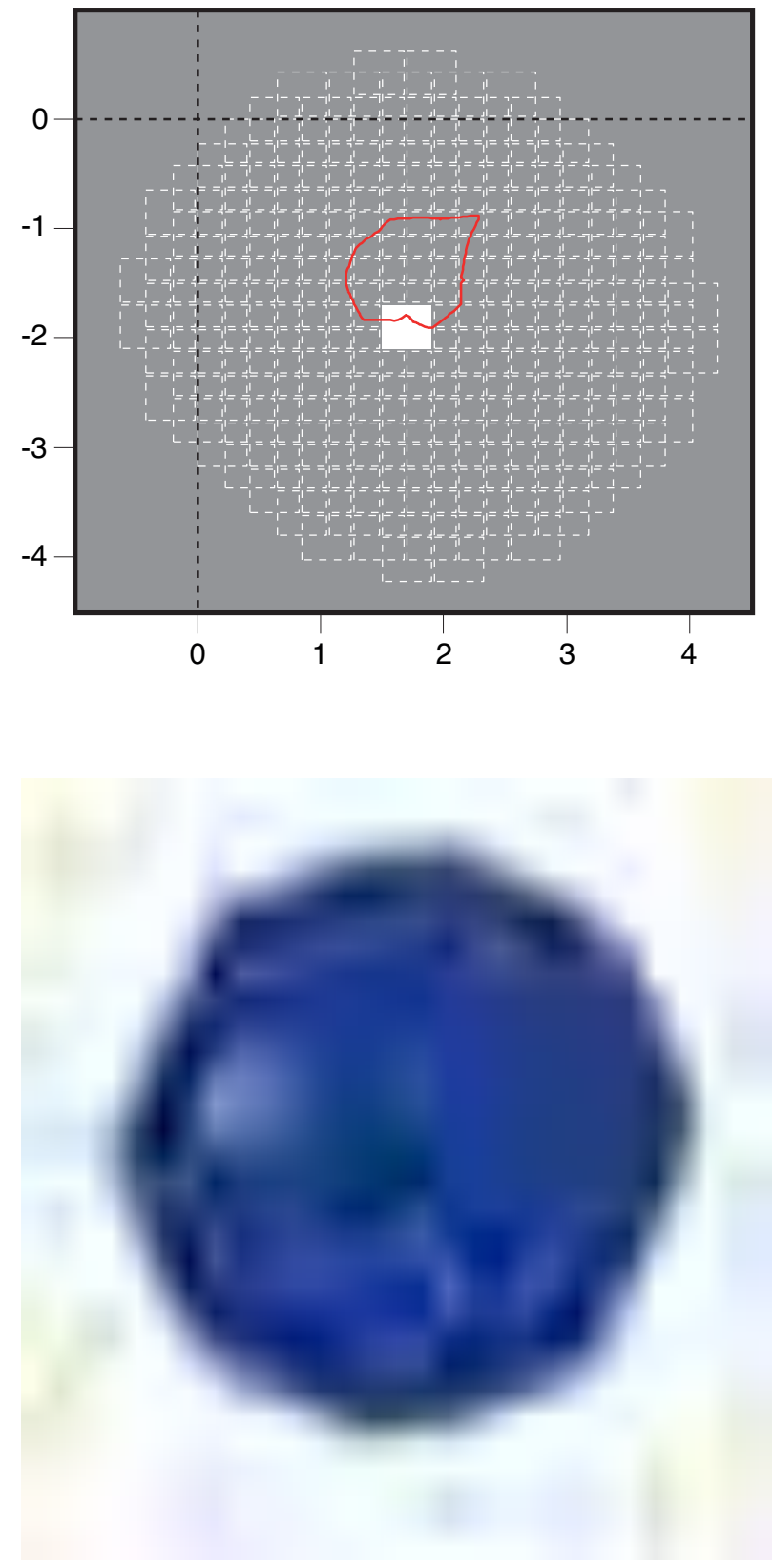

Figure 2 - Mapping grid

$\mathrm{X} / \mathrm{Y}$ axis mark the visual degree. The position of the fixation point was at $0 / 0$. The white square symbolises the actual probe in one example position. The red line shows one example receptive field. All 178 positions the probe could appear at, are marked as a rectangle with dotted lines. Note that the positions the positions of the probe are all overlapping.

Figure 3 - Visual Field Map

Example of a visual field map for one recording site. $\mathrm{X}$ - and $\mathrm{Y}$ - axis are in the visual angel. $0 / 0$ is the position of the fixationpoint. Blue colors mark low neuronal responses, red colors high neuronal response rates. The black contour depicts half the maximum response and defines the outline of the receptive field. The cross marks the position with the maximum response.

\subsection{2 - Attentional Task}

The spatial cueing task we developed for this study was based on the task used previously by Womelsdorf et al. (2006): After foveating the fixation point the monkey had to touch a lever, which was attached to the monkey chair, in order to start an experimental trial. A static random dot pattern (RDP) was then presented for $440 \mathrm{~ms}$ on the screen, indicating the spatial position of the valid stimulus (target) for this trial. After a brief delay of $130 \mathrm{~ms}$ two random dot stimuli appeared on the screen, which were 1.5 degrees in diameter. The monkey had to indicate a 
small direction change of 45 degree in the target stimulus while ignoring the other stimulus (distractor) (Fig. 4). The spatial layout of the two stimuli was constant over all experimental sessions. The centre of stimulus 1 was placed at the position $-1.35 /-1.65$ degree on the screen. At this position the stimulus would cover most of the receptive fields optimally. Stimulus 2 was placed at the same eccentricity but such that no receptive field would be covered by the stimulus (1.95/0.95 degree) (see Fig. 13 for correct representation of stimuli in relation to receptive fields). The distance of the two stimuli centroids was 4 degrees. The space the two stimuli cover was adjusted so that both could be positioned into a hypothetical MT receptive field. The lengths of the trials were taken from a flat distribution and could vary between $1000 \mathrm{~ms}$ and $7000 \mathrm{~ms}$. The monkey got rewarded for successfully indicating a direction change in the target stimulus within a response window of up to $500 \mathrm{~ms}$ after the response event. Trials in which the monkey reacted before or after the response window or saccaded outside the fixation window of 1 degree radius, were not rewarded. In one condition of the experiment ("with-probe" condition), we presented an additional probe stimulus simultaneously to the target and distractor stimulus. While the monkey was attending to the target stimulus a small random dot pattern ( 0.75 degrees diameter) flashed up for 200 ms at various position on the screen (Fig. S10). The monkey had to ignore this stimulus. In each experiment we presented two different directions in the RDPs. Direction 1 and direction 2. Direction 2 was always 180 degree opposite of direction 1. Target and distractor moved both move into the same direction or in opposite directions. All together we had four different direction pairs: 0/180, 45/225, 90/270, 137/315 degrees.

In the attentional conditions of the task, the monkey was instructed with a cue to attend to either the stimulus outside or inside the receptive field. In the attentional control conditions, the sensory stimulation was the same as that of the attentional condition, but no cue stimulus was shown. Instead the monkey had to attend to the fixation point and indicate a small luminance change in the fixation point. In addition, we used one baseline condition, in which only the fixation point was present on the screen. The monkeys task was again to indicate a luminance change in the fixation point. The different types of conditions were indicated to the monkey with different colored fixation points. Attentional conditions were marked with a red fixation point, all other condition had a grey fixation point. 
Alltogether we had the following conditions: attentional condition inside ("In"), attentional condition ("Out"), attentional control condition and baseline condition. All conditions were tested with all direction pairs.

Different conditions were presented block-wise. In the majority of our recording sessions the blocks were structured as followed: 25 successfully completed trials ("hittrials") for the attentional conditions in which the direction combination of the stimuli could vary, but the attentional target was constant (either attending inside or outside the receptive field). 10 hittrials of intermixed baseline and attentional control conditions. For the attentional control conditions the combination of direction could vary. Despite the block design, we presented the cue stimulus for the attentional conditions in every trial.

Figure 4 - Timecourse of a trial in the attentional condition ("without Probes")

The trial starts when the monkey holds the lever and fixates on the fixation point (Fixation). A static random dot pattern appears and indicates the spatial location of the stimulus, which the monkey should attend to in the run of the trial (Cue). After a brief delay (Delay), two moving random dot pattern appear (Stimulus Onset). The monkey has to indicate a small direction change in the target stimulus (in this example, the upper stimulus) by releasing the lever. The response event can take place any time between 600 and $7000 \mathrm{~ms}$ after trial onset (Response Event). For the attentional control condition, the timecourse layout is exactly the same, with the difference of no cue being presented.

Note that this graphic is only a sketch, which represents the actual sizes and distances only approximately. For a correct representation of size, position of the receptive fields in relation to the stimuli and distance between the two stimuli please refer to picture 12 and 13. 


\section{6 - Data Analysis}

All calculations were performed with custom written Matlab Scripts (The MathWorks, Inc).

We measured the neuronal responses of each electrode from successfully initiated trials until behavioural relevant events occurred (i.e. color changes of the fixation point or direction changes of the attended stimulus). Trials which were terminated due to fixation errors or when the lever was released outside the response time window were not analysed. For determining the effects of spatial attention we analysed only the successful trials of the conditions, in which both stimuli were moving in the same direction. To be included in the analysis a dataset had to have at least 8 successful trials in all valid conditions.

\subsection{1 - Criteria}

We analysed neuronal responses in the period of $600 \mathrm{~ms}-1600 \mathrm{~ms}$ after trial onset for the attentional trials and attentional control trials. For the baseline condition we chose a period of $100-700 \mathrm{~ms}$ after trial onset. We calculated the mean neuronal response rate for each condition by averaging over all trials of the condition in the analysis period. To determine the responsiveness of the neurons we compared the mean neuronal response rates in the baseline condition to those of the attentional control conditions. In the baseline condition only the fixation point was on the screen and the monkey had to detect a subtle luminance change in the fixation point. In the attentional control condition, the monkey had to report a luminance change in the fixation point as well, but we simultaneously presented the two stimuli on the screen. The stimuli would either move both in direction 1 or direction 2. To be recognized as responsive, a neuron had to show a doubling of the mean neuronal response rate in at least one attentional control condition compared to the baseline condition. The mean of the attentional condition and the baseline condition had to be significantly different (two-sample ttest, $p<0.05$ ). Neurons, which fulfilled the requirements, would be included in the spatial attention analysis. 
We additionally tested the responsive neurons for direction selectivity. To be considered direction selective, mean neuronal responses in the condition attentional control condition direction1 (acc 1) and attentional control condition direction2 (acc 2) had to show a difference in the response of 3 times the mean neuronal response rate. Data, which passed this requirement, would be considered as direction selective. For the subpopulation of direction-selective cells we calculated the directional index according to:

$$
\mathrm{DI}=(\operatorname{acc} 1-\operatorname{acc} 2) /(\operatorname{acc} 1+\operatorname{acc} 2)
$$

or vice versa (Snowden et al., 1992). We found only a small percentage of our cells were direction selective, therefore we did not focus our analysis on these cells. Note that it was independent of direction selectivity as to whether a cell would be included in the spatial attention analysis.

\subsection{2 - Analysis}

To determine the strength of the attentional modulation we calculated the attentional index for the conditions in which the monkey had to attend inside the receptive field ('In') and when the monkey had to attend to the stimulus outside the receptive field ('Out'). After subtracting the neuronal response rate of the baseline condition from the neuronal response rate of the attentional conditions, we determined the attentional index according to

$$
\mathrm{Al}=(\ln -\text { Out }) /(\ln +\text { Out })
$$

We calculated one attentional index for every neuron, which was included in the analysis. For neurons, which were direction selective we calculated the Al for the preferred direction. For neurons, which were not direction selective we calculated the attentional index for the direction in which the neuronal response rate was higher. The difference did not need to show significance.

To control whether the attentional modulation was multiplicative, we additionally computed the attentional index for the null direction of the neurons. We tested 
whether the two subpopulations (preferred direction, null direction) were significantly different with a paired ttest. If the effect follows the multiplicative scaling, the average difference of the attentional indices of both subpopulations should not differ significantly $(p<0.05)$.

To identify an additive effect, we calculated the absolute difference in firing rate between the conditions 'In' and 'Out' for the preferred direction and null direction. If the attentional effect is additive, the absolute difference in firing rate should be similar for the preferred and the null direction. We tested whether the two populations were significantly different with a paired ttest $(p<0.05)$.

\section{7- Eyeposition}

While recording a spatial attentional task in V1, ensuring a stable eyeposition is extremely important. Receptive fields in V1 are small. The average size lays in the magintude of 1-2 degrees (e.g. Gur \& Snodderly, 2007). And a high fluctuation or a systematic shift of the eyepostion could lead to a corresponding shift of the retinotopic receptive fields. Because of the receptive fields size in $\mathrm{V} 1$, the probability that due to a shift in eyeposition the optimal stimulation of the neuron by the stimulus is not given anymore is very high. We monitored the eyeposition of the monkey's left eye with a video-based eye-tracking system (Thomas Recordings, Gießen, Germany). The eyepositions were recorded at $230 \mathrm{~Hz}$, digitized and stored at $200 \mathrm{~Hz}$. Eyefixation was controlled throughout the whole experiment. The fixation window was always 1 degree radius around the fixationpoint. Any saccade outside this fixation window during a trial would lead to the abortion of the ongoing trial.

We analysed the monkeys eyepositions for all hittrials in the different experimental conditions in order to find out whether or not there is a systematic shift in the monkeys eyeposition depending on the attentional condition. For the time window of the analysis period (600-1600 ms after stimulus onset) we calculated eyepositions in the baseline condition (only fixation point is present), for the conditions in which the monkey had to attend inside the receptive field (attentional condition 'In') and the conditions in which the monkey had to attend outside the receptive field (attentional condition 'Out'), independent of the directions 
presented. We calculated the average eyeposition of all hittrials of the aforementioned three conditions and averaged over all data sets. We plotted the results in a coordinate system, relative of the $0 / 0$ degree position.

\section{8 - Behavioral Control}

We measured the monkey performance, by calculating the mean hittrate and mean reaction times. We calculated these values for the conditions, on which we based our spatial attention analysis. We averaged across experimental sessions. Because we were rather interested in how many of the errors were due to a misallocation of attention, we subtracted the number of trials which were aborted due to a fixation error from the complete number of trials before calculating the mean.

\section{Results}

\section{1 - Confirmation of recording site}

We analysed the receptive field positions for every recording site. At a mean distance of 1.77 degrees eccentricity ( $p<0.00005, S D=0.3153$ ) relative to the fixation point the receptive fields showed a mean size of 0.3 ( $p<0.00005$, $S D=0.1258$ ) degrees (Fig. 5). The receptive field size is in accordance with literature values (Hubel \& Wiesel, 1974; Gattass et al., 1987; Gur \& Snodderly, 2007). Considering the size our array was covering on the surface of the cortex (Gattass, 1987), a correlation of size and eccentricity was not expected and not apparent in our data. We did however see a clear correlation of eccentricity and position of the centroid of the receptive fields. We found that the eccentricity increases the further away from the fovea $(0 / 0)$ the recording site is (Fig. S3-9). 

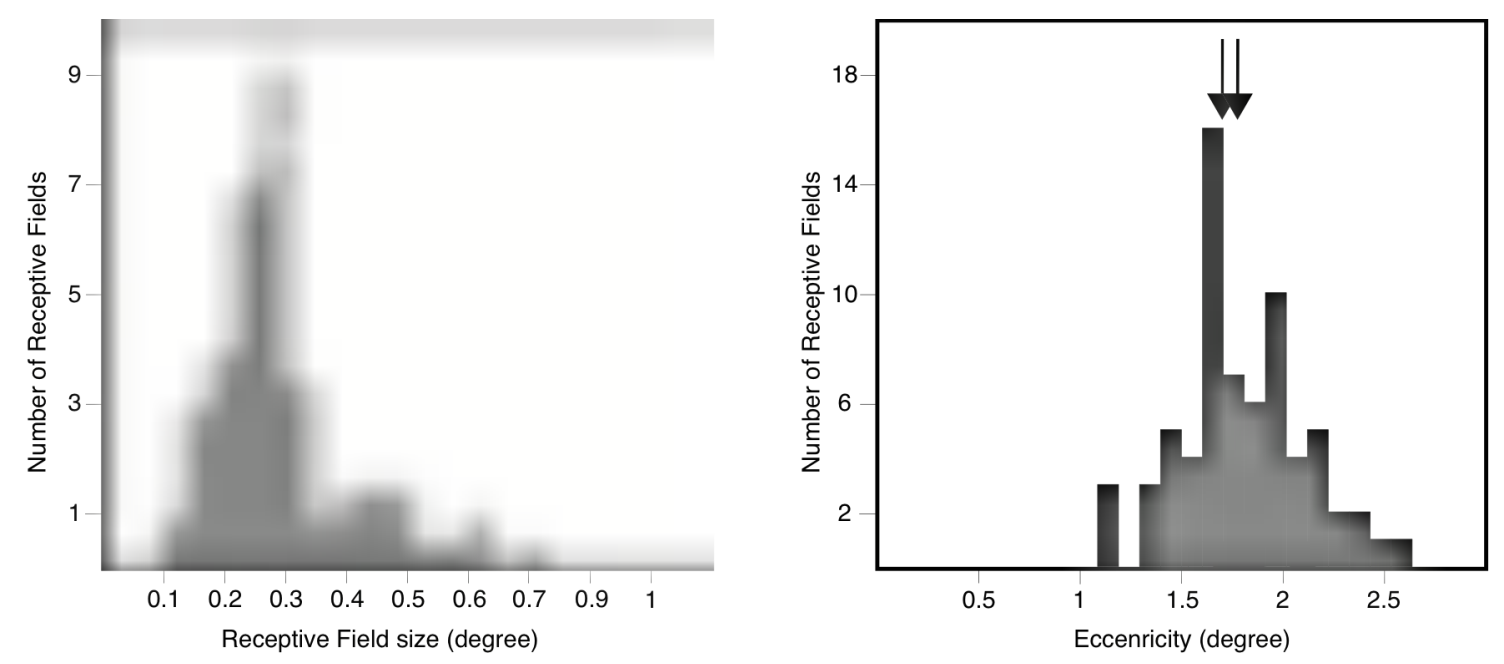

Figure 5 - Distribution of receptive field size and eccentricity Left - Frequency histogram of receptive field size in visual degree.

The $x$-axis denotes the size in visual degree; the $y$-axis denotes the number of receptive fields with a given size. The black arrow indicates the mean ( 0.29 degrees), the grey arrow indicates the median (0.27).

Right - Distribution of distance of the receptive centroids from the fovea.

The $x$-axis denotes the eccentricity of the receptive field centroids from the fovea in visual degree; the $y$-axis denotes the number of receptive fields. The black arrow indicates the mean distance (1.77 degree); the grey arrow indicates the median distance (1.67).

\section{2 - Attentional Conditions}

\subsection{1 - "with-probes" condition}

To analyse the spatial attentional modulation in V1, we compared the condition of attending the stimulus inside the receptive field against attending the stimulus outside the receptive field for those trials where both stimuli were moving in the same direction. For analysing the spatial attentional modulation we did not take directional tuning into account, but units only had to show responsiveness towards the stimulus (see Materials and Methods). Of 2208 neurons, which initially were recorded for this condition, 844 remained in the final analysis. Of the 1364 neurons, which were excluded from the final analysis, the majority (980 neurons, $71.8 \%$ ) were excluded because they did not show a sufficient response to the 
stimuli. $384(28.15 \%)$ neurons did not have a minimum of 8 successful trials in the valid conditions and were therefore excluded from the analysis. (Fig. 6) For every neuron we determined the directionality. We defined directionality as a 3 fold difference in the neuronal response rate to stimuli differing by 180 degree for the attentional control conditions (acc 1 VS acc 2). Out of 844 units, which were responsive to the stimulus, 48 neurons showed a significant directional preference (mean direction selectivity index: 0.72 ( $p<0.00005)$ ) (Fig.7).

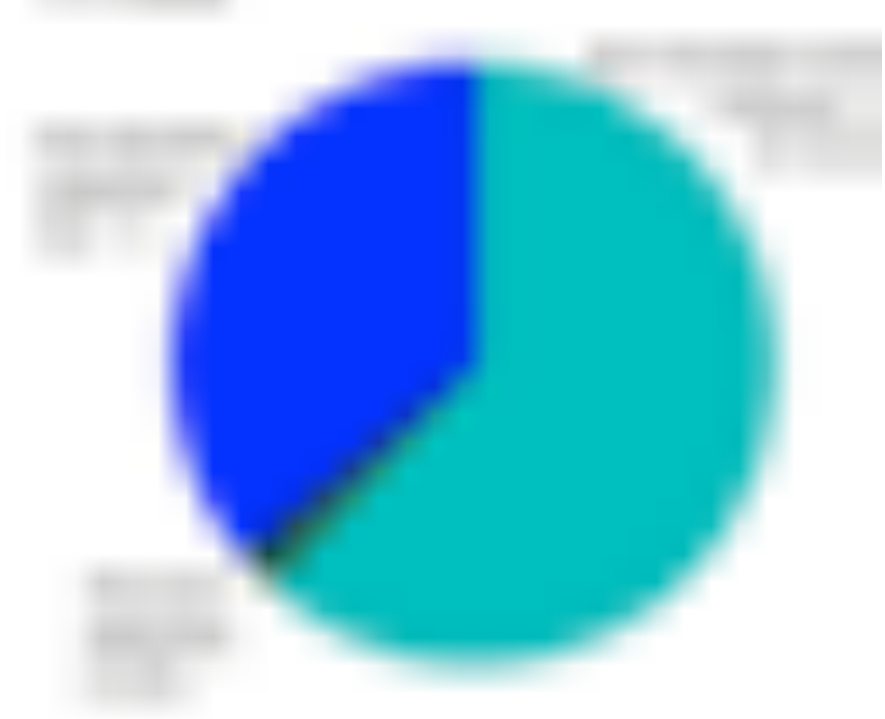

Figure 6 - Number of neurons in the analysis ("with probes" condition).

The complete dataset consisted of 2203 neurons. Of those, $61.9 \%$ (1364 units) were not included in the analysis (cyan), 36\% (796 units) were not direction selective (blue) and $2 \%$ (48 units) were direction selective (green).

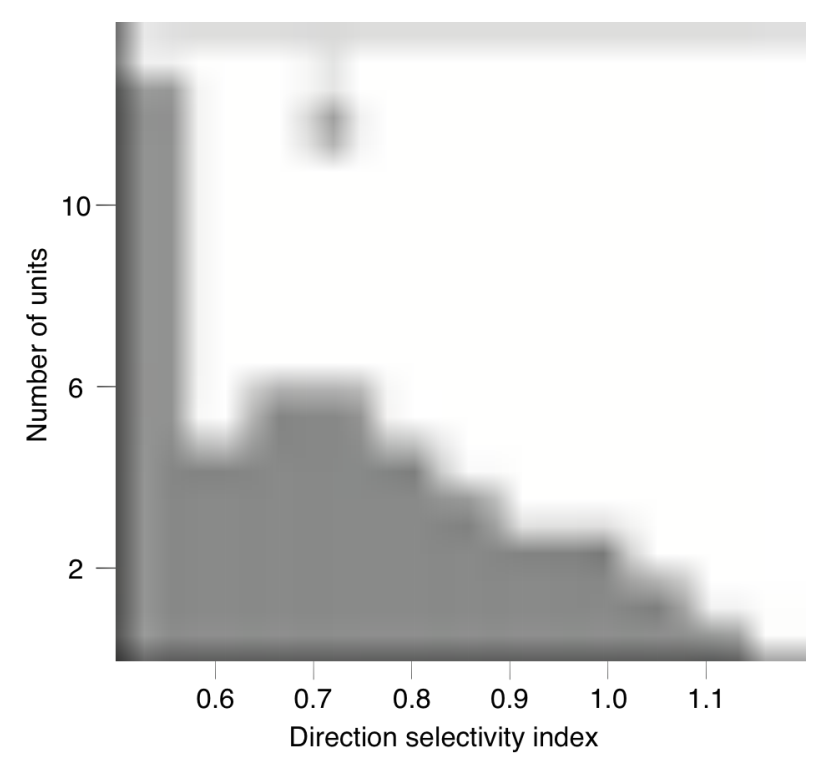

Figure 7 - Histogram of direction selectivity indices for subpopulation of direction selective units The $x$-axis denotes the direction selectivity index; the $y$-axis denotes the number of units. The black arrow indicates the mean $(0.72)$, the grey arrow indicates the median (0.7). 
We calculated the attentional indices for neurons, which showed a significant responsiveness towards the stimuli. These neurons showed a small, but significant positive modulation of 3.5\% ( $<$ < 0.00005) (Fig. 8). We separated the population of neurons into neurons, which showed direction selectivity and neurons, which showed no direction selectivity. For neurons, which showed no direction selectivity we find a significant attentional modulation of $4.1 \%(p<0.00005)$ (Fig. 9)

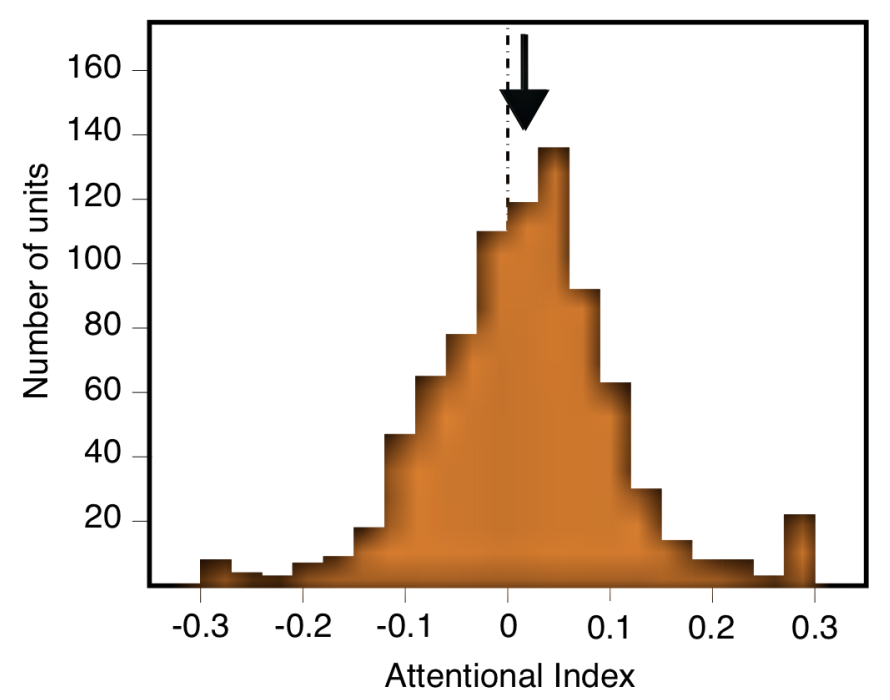

Figure 8 - Distribution of attentional indices for all neurons, which were responsive to the stimulus ("with probes" condition)

On the $y$-axis the number of neurons is depicted, on the x-axis, the attentional index. The dashed black line indicates 0 on the $x$-axis. A black arrow indicates mean values, medians are indicated by a grey arrow. For visibility reasons, we restricted the $x$-axis to the values of $0.3 /$ 0.3 .

The average attentional modulation of the population is $3.5 \%(p<0.00005)$ (black arrow). The median of the modulation is $3.41 \%$. There were 19 values larger then 0.3 and 6 lower then 0.3 .

The direction selective neurons showed a modulation of $-5.95 \%$, but the modulation was not significant ( $p>0.05)$ (Fig. 10). In order to evaluate whether the mean attentional effect for the direction selective subpopulation differs to the mean effect of the entire population we used bootstrapping to estimate the confidence interval of the mean attentional index of the complete population. We did a bootstrap analysis with 10000 repetitions. We found that the mean attentional index of the direction selective subpopulation (mean attentional index $=-0.031$ ) 
lays outside the confidence intervals (ci_lower $=-0.015$, ci_upper $=0.048$ ) for the means extracted with the bootstrap analysis. This indicates that the result for the subpopulation could not come from randomly sampling attentional indices from the whole population.

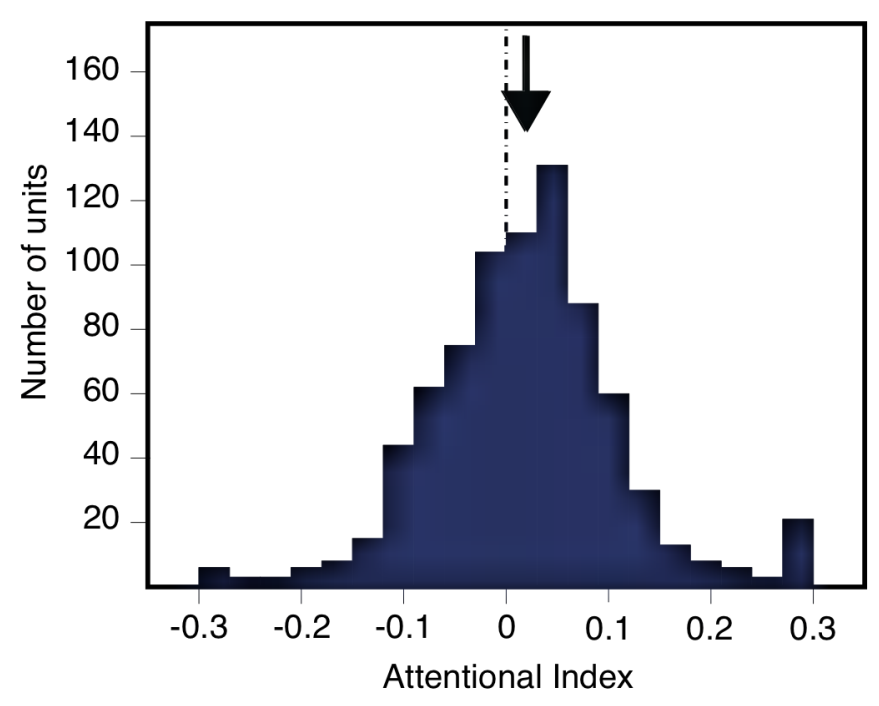

Figure 9 - Distribution of attentional indices for neurons, which were not direction selective ("with probes" condition).The mean attentional modulation is $4.1 \% \quad(p<0.00005)$ $($ median $=(3.72 \%))$. There were 18 values larger then 0.3 and 4 lower then -0.3 .

Description of the axis see figure 4.

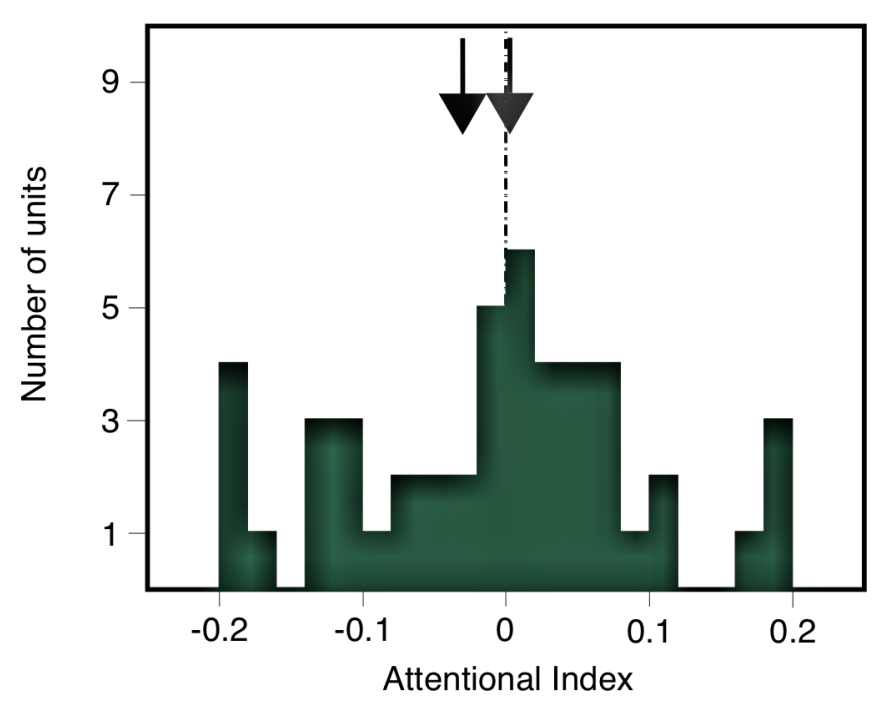

Figure $10-$ Distribution of attentional indices for neurons, which were direction selective ("with probes" condition).

The mean attentional modulation is $5.95 \% \quad(p>0.05) \quad$ (black arrow). A grey arrow indicates the median modulation $(0.55 \%)$. For visibility reasons, we restricted the $x$-axis to the values of $0.2 /-0.2$. There were 3 values larger then 0.2 and 3 lower then -0.2. The minimum value for the attentional index in the population was -0.52 ; the maximum value was 0.303 .

We tested whether the data showed an attentional modulation, which is multiplicative or, alternatively, additive. Multiplicative attentional modulations could be found in a number of visual areas such as MT or V4 (Maunsell \& McAdams, 2001; Treue \& Maunsell, 1996). For V1, however, there are a number of 
evidences, that the attentional effect is additive, rather then multiplicative (Boynton, 2011; Thiele et al., 2009). For identifying the attentional mechanism, we analysed the subpopulations of direction selective and non-direction selective neurons separately.

For direction selective cells we compared the attentional index of the preferred direction against the attentional index of the null direction in a paired ttest. If the modulation was multiplicative the two populations should not differ significantly from each other. We found however, that the two populations were significantly different from each other $(p<0.00005)$. For neurons, which did not show a significant directional selectivity, we defined the preferred direction as the direction, which would lead to the higher absolute neuronal response rate and vice versa. We compared the two populations in a paired ttest and found a significant difference $(p<0.00005)$.

To identify an additive effect, we again divided the population of neurons into the aforementioned subpopulations. We calculated the absolute difference in firingrate for the conditions 'In' and 'Out', once when both stimuli went in the preferred direction and vice versa. If the attentional effect is additive, the absolute difference in firingrate should in both cases be similar. Therefore the paired ttest should show no significant difference between the two conditions of the subpopulations. For the two conditions of the direction selective cells (preferred direction versus non-preferred direction) we found a significant difference $(p<0.00005)$. We found the same result when testing the subpopulation of the nondirection selective cells $(p<0.00005)$.

\subsection{2 - "without-probes" condition}

We conducted the same analysis for the attentional condition in which no probe stimuli were presented. Of 480 cells, which went into the analysis, 35 remained after applying the forementioned criteria. All of the 445 cells, which did not qualify for the analysis, were removed because they did not fulfil the response criterion. Of the 35 remaining cells, none were direction selective (Fig. 11). We again 
calculated the attentional indices for neurons, which showed a significant responsiveness towards the stimuli. These 35 neurons did not show a significant attentional modulation (mean $\mathrm{Al}=-2.84 ; \mathrm{p}>0.05)$ (Fig. 8).

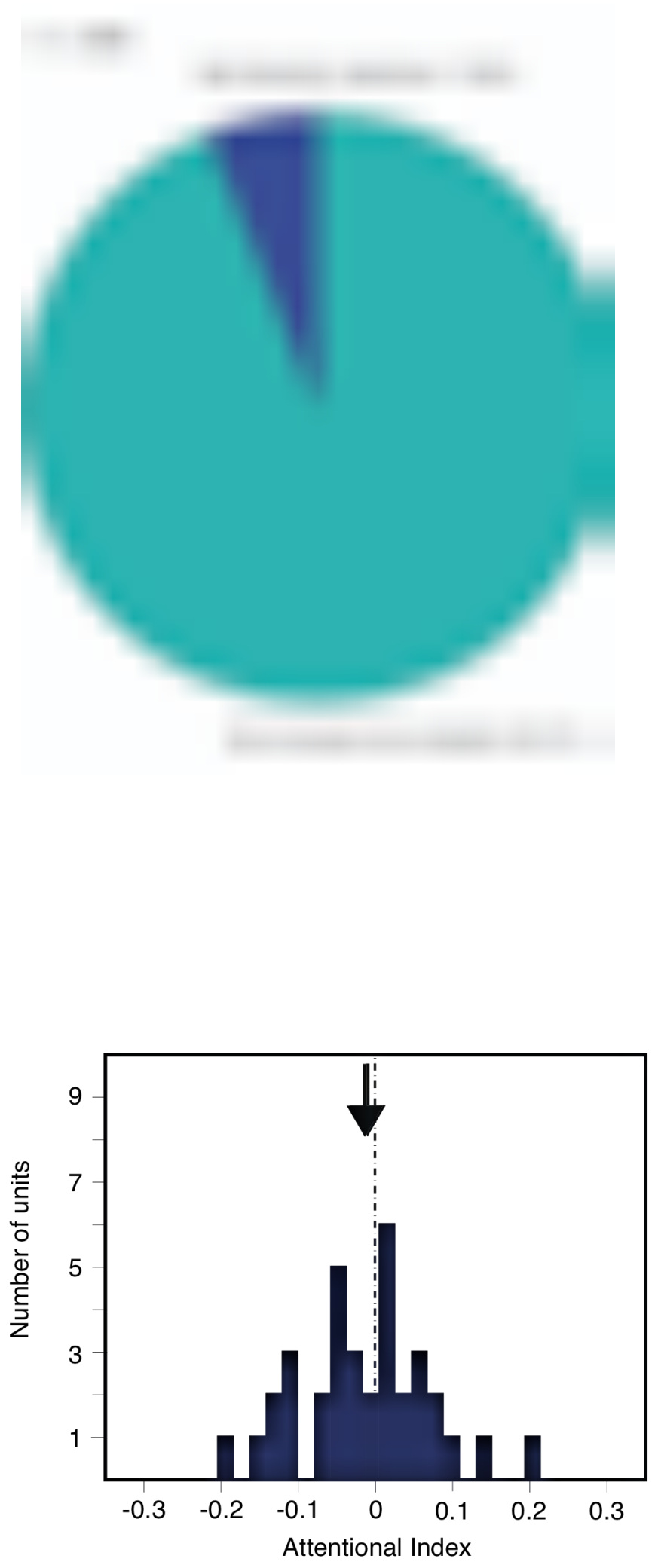

Figure 11 - Number of neurons in analysis ("without probe" condition)

The complete population had the size of 480 neurons. Of those, $92.7 \%$ (445 units) were not included in the analysis (cyan). The remaining 7\% (35 units) were not direction selective (blue).

Figure 12 - Distribution of attentional indices for all neurons, which were responsive to the stimulus ("without probes" condition).

On the $y$-axis the number of neurons is depicted. On the $\mathrm{x}$-axis, the attentional index.

The mean attentional modulation is $-2.84 \% \quad(p>0.5)$ (black arrow). A grey arrow indicates the median modulation (-1.98 \%). 


\section{3 - Eyepostion}

We computed eyepositions for the attentional experiment ("with probes"). We computed the average eyeposition for the time of the relevant analysis period for the attentional conditions of attention inside the receptive field and outside the receptive field. Additionally we computed the mean eyeposition for the fixation only condition in the same time window. We find a shift of 0.09 degrees between the two fixation conditions $(p<0.0005)$. The mean distance between the fixation only and attentional condition 'In' is 0.165 degree $(p<0.0005)$. The mean distance between the fixation only and attentional condition 'Out' is 0.16 degree $(p<$ 0.000005) (Fig. 12). A shift of the eyeposition depending on the condition is to be expected. However, if we transfer these shifts to the position of the receptive field in relation to the stimulus we can see that the shifts in the eyeposition would have led to a very small displacement of the stimulus depending on the experimental condition (Fig. 13). The majority of the receptive fields seemed to be covered well in both attentional conditions. Although we did not test for the effects of the eyepositions quantitatively, we are very confidents in assuming that the magnitude of the displacement does not have a significant effect on the neuronal responses.

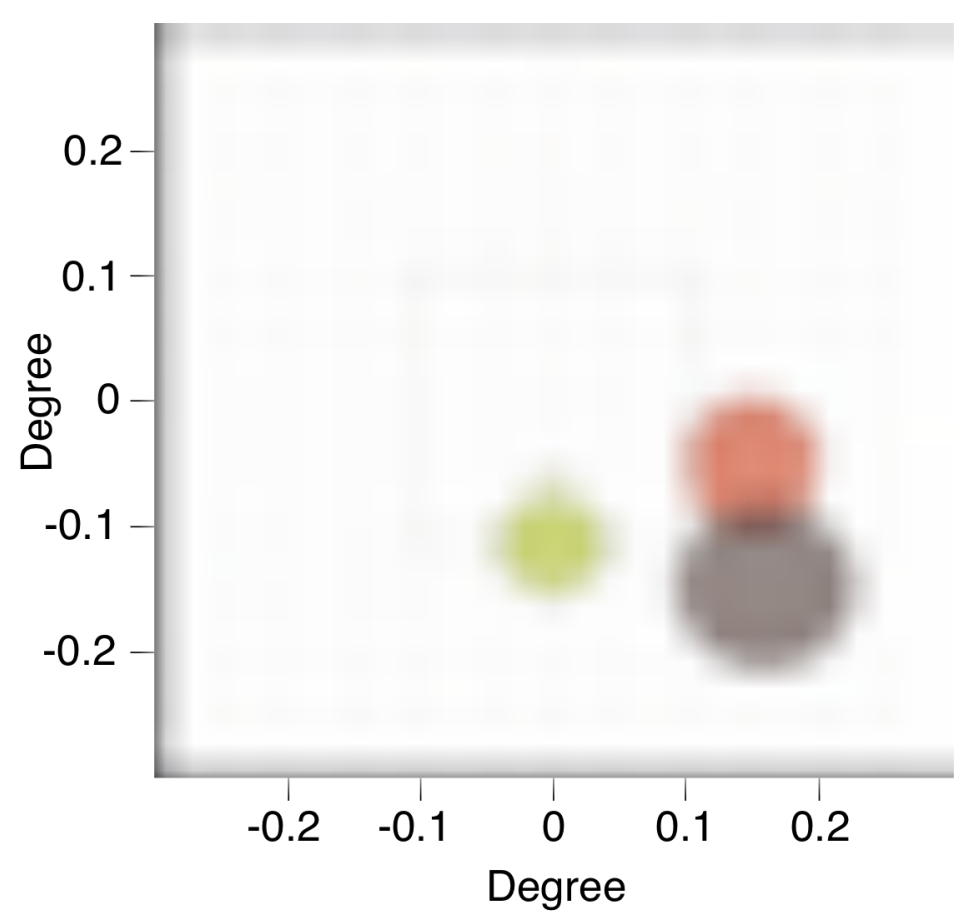

Figure 12 - Average eyeposition for attentional condition 'In'/'Out' and Fixation Only condition.

The $\mathrm{x}$ and $\mathrm{y}$ axis are depicted as visual angle in degree. The center of the circles mark the mean $\mathrm{x} / \mathrm{y}$ positions of the eyepositions, the radius is the square root of the standarddiviations of $x$ and $y$. Yellow is the fixation only condition, brown attentional condition 'In', red attentional condition 'Out'. The dotted square marks the outline of the fixationpoint on the screen. 


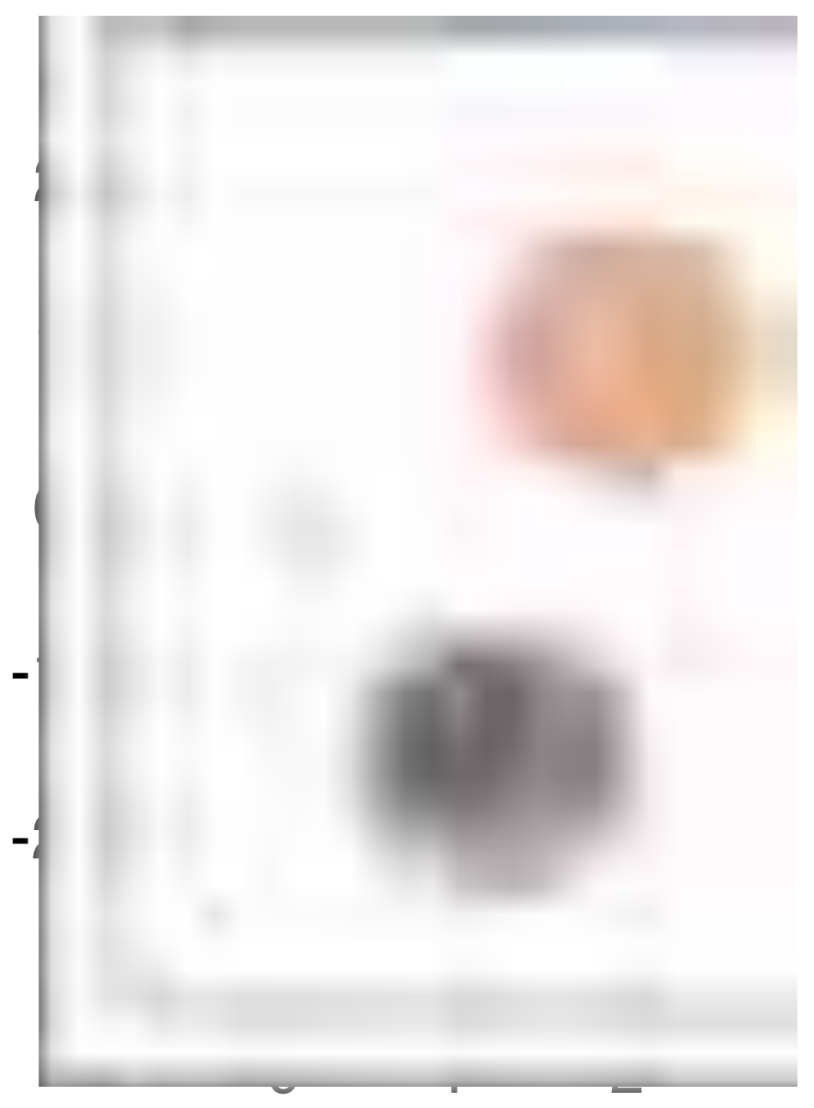

Figure 13 - Stimuli positions in relation to receptive fields and average eyepositions.

$X / Y$ axis are in visual degree. Circles indicate the stimuli positions in the visual field (red - outside stimulus; brown inside stimulus). Grey lines indicate receptive field size and positions; in red four receptive fields are highlighted as random examples (see figure $\mathrm{S4}$ ). The black dashed square indicates size and position of fixation point. Small dots at the fixation point indicate average eyepositions for different attentional conditions (red - attention outside, brown - attention inside, yellow fixation only (see also figure 12)).

\section{4 - Behavioural performance}

After subtracting trials which were aborted due to fixation breaks, we calculated the mean hitrate and mean reaction time averaged over all attentional and all attentional control conditions. Mean hitrate for the control condition was $99.69 \%$ $(S E=0.2155, S D=1.46)$. Mean hitrate for the attention conditions was $70.42 \%$ $(S E=1.2956, S D=12.43)$. Mean reaction time for the attentional control condition was $410.82 \mathrm{~ms}(\mathrm{SE}=0.79, \mathrm{SD}=24.18)$. Mean reaction time for the attentional conditions (attending inside and outside of the receptive field) was $496.53 \mathrm{~ms}$ (SE $=0.8712, \mathrm{SD}=39.73$ ). Differences in the mean performance and mean reaction time between the attentional control conditions and attentional conditions were significant $(p<0.0005)$. There was no significant difference between mean hitrates and mean reaction time for the attentional condition when the monkey attended inside the receptive field or outside the receptive field $(p>0.05)$. 


\section{Discussion}

In this project we were interested in contributing to solving the still open question of how receptive field shift, induced by spatial attention, in area MT, come to pass. The foundation for our proceedings were laid out by two hypothesises concerning this modulation in spatial selectivity. Maunsell and McAdams (2001) suggested that receptive field shifts in higher visual areas, such as area MT, spring from multiplicative attentional effects in lower area neurons, specifically in the primary visual cortex V1. Alternatively Womelsdorf and colleagues (2008) offered an alternative hypothesis, which states, that while the receptive field shifts in area MT might be due to a multiplicative effect, this effect does not necessarily have to be inherited from V1 directly. It can just as well be a characteristic of synaptic inputs of area MT neurons. To investigate the questions which of these two hypothesises is more likely to offer an explanation for the receptive field modulation in area MT, we recorded from neurons in V1 while the monkey did a spatial attention task. The spatial attention task was based on the spatial attention task of the Womelsdorf study. The task design, however, might provide a specific arrangement of the stimuli, which might promote attentional effects in V1. We tested this hypothesis by comparing attentional modulations of two versions of the task. One resembled the task design of the Womelsdorf study in its details ("with-probe" condition), in the alternative version however, we removed the distracting probe stimulus and only presented the two random dot pattern ("without-probe" condition).

To identify a spatial attentional effect we analysed neuronal responses of neurons, which showed a significant sensory response towards our stimuli. Additionally we analysed the direction selectivity of those neurons. Only a small percentage of cells showed significant direction selectivity. Snowden and colleagues (1992), who tested cells for their direction selectivity, showed that $32 \%$ of their tested neuronal population in V1 showed a significant direction selectivity. We find only $2.1 \%$ of our neuronal population showing a significant direction selectivity. The most likely explanation for this is, that due to our diminished signal, most of the neuronal signals were too noisy to pass the high criterion we had set, to be recognized as direction selective. Furthermore it is to note, that multiunit activity reflects data, which is pooled over the responses of several neurons. Although, due to the 
column architecture of the area, it is highly likely that cells which are in close proximity to each other, share certain features, as for example direction tuning, the data nevertheless reflects data from a pool of neurons and will therefore not show such a defined tuning as data from a single neuron (Super et al., 2005). An alternative explanation to the lack of direction selective cells could also be, that the electrodes of our array did not penetrate the layer 4 in V1, which holds the majority of direction-selective cells (Livingstone, 1998). Opposing this explanation, however, is our receptive field size. Gur \& Snodderly (2007) found for receptive fields in layer $4 \mathrm{~B}$ receptive field width ranging from 0.2 to 0.29 degrees. Receptive fields in other layers ranged around 0.1 (layer 3) and 0.48 (layer 6) degree. Our receptive field size are 0.29 degree on average, which matches their finding in receptive field size for layer 4 . Nevertheless, we can not state with all certainty, that our electrodes did in fact record from layer 4, as we did not test for other characteristics cells in this layer show (waveforms, signal-to-noise ratio (Livingstone, 1998)).

For both versions of the task designs, we divided the population of the cells, which showed a sensory response into direction selective and non-selective cells. We calculated the directional index for the direction selective cells and found a relatively high level of direction selectivity (direction selectivity index: 0.7237 (mean), 0.709 (median) $(p<0.00005))$. Snowden et al., 1992 found a direction selectivity index of 0.44 for a population of V1 neurons. Our subpopulation shows a much higher direction selectivity. Movshon et al. (1996) could show that V1 cells, which project directly to a MT cell show a high direction selectivity (direction selectivity index $=0.96$ ). We might therefore take this as careful indication that at least some of our direction-selective neurons might also project directly to a MT cell.

We calculated the attentional index for the subpopulations of direction and nondirection selective cells. For the data from the "with-probe" condition, we found a small but significant attentional modulation of the whole population of $3.5 \%$ increase of neuronal responses. We also found a significant modulation of $4.1 \%$ for the non-direction selective cells, but no significant modulation for the direction selective cells. Considering the established strong link between projections of 
direction-selective cells in V1 and MT, this result is surprising. One reason for this might be, that our sample size of 48 neurons was too small for the results to be representative. But because we did not do any statistical power analysis, we can only speculate on this. If however, we take the result as genuine, one implication might be, that while tuning properties are inherited from lower areas to higher areas, attentional modulation might not. This stands in contrast with the implication of the hypothesis of Maunsell \& McAdams (2001), who suggested, as a consequence of their multiplicative scaling model, attentional effects are not a topdown influence from higher areas, but are also projected from lower to higher areas along the hierarchy. If that would hold true for our data, we should see an attentional modulation particularly in the direction selective neurons. Of all neurons in our population, they are the most likely candidates for projecting directly to area MT. We see no effect in the population of direction selective neurons, therefore our data does not support the hypothesis of Maunsell \& McAdams.

For the data from the "without-probe" condition we did not find any significant attentional modulation. This however can be due to the quality of the signal. More the $95 \%$ of the recorded data did not show a sufficient responsiveness towards the stimuli. If, however, we compare our results for the "with-probe" condition to a study in which they also presented two stimuli -one inside, one outside the receptive field, both in close proximity- but no additional visual input (Luck et al., 1997), we can see that unlike our results, they could find no attentional effects in V1. This comparison can be understood as an additional evidence, that attentional modulation in V1 is dependent on the stimulus arrangement and task demands.

In comparison to other cortical areas, neurophysiological data showed only small attentional modulations for $\mathrm{V} 1$. The effect ranging in the magnitude of $5-8 \%$ (Olshausen \& Field, 2005; Maunsell \& Cook, 2002). Our results are slightly lower then the effects strength on average, but still in the approximate order of magnitude.

We also analysed the characteristics of the observed attentional effect. For a number of different areas it could be shown, that the attentional modulation are often multiplicative (Maunsell \& Cook, 2002; Maunsell \& McAdams, 2001). For V1 
however this does not seem to be the case. fMRI studies in humans showed an additive modulation rather then multiplicative (Boynton, 2011). Neurophysiology data from monkeys could confirm this result. Thiele and colleagues (2009) found that spatial attention had an additive rather then a multiplicative effect on the contrast response function for $\mathrm{V} 1$ neurons. In light of these findings, we analysed our data as to whether the modulation mechanism was additive or multiplicative. For testing the data for a multiplicative effect, we divided the subpopulations of direction selective cells depending on their preferred directions. We computed the attentional indices for all cells in those two groups and tested whether or not they were significantly different from each other. We found that for the direction selective cells the attentional indices of the two groups were significantly different from each other. We found the same result for the non-direction selective cells. If the attentional effect was multiplicative, we should not find a significant difference between the groups, because the attentional effect should be of the same magnitude independent of the direction presented to the neuron (Maunsell \& McAdams, 2001).

For analysing whether or not the data would alternatively show an additive effect of the attentional modulation, we defined the attentional modulation as absolute difference of the firing rate between attention 'In' and attention 'Out'. We computed the additive attentional modulation for the preferred and null direction for every neuron. We tested the additive attentional modulation for the subpopulation of direction selective cells with a paired ttest. If the attentional effect is additive the absolute difference in firing rate between the two directions for the two attentional states should be the same; independent of the preferred direction, because the additive modulation adds a constant value of spikes to the firing rate, independent of the direction of the presented stimulus. We found that for the subpopulation of direction selective cells, the additive attentional modulation was significantly different $(p<0.00005)$. The same is true for the non-direction selective cells.

One explanation that we don't observe a clear result, which supports one over the other attentional mechanism, could be the diminished quality in the signal (see Materials and Methods). We did not test specifically for the signal-to-noise ratio as a qualitative measure for our data, but the rapid decline of recording sites, which 
showed responsive data, over time (see Results 2.3.1), can be taken as an indication for a high level of noise in our data. The level of noise would specifically have an effect on the observation of an additive effect. In the additive effect, a constant value of spikes is added to a baseline firing rate. If the level of noise in the signal is high, the constant factor will become obscured within the noise.

We could show that neurons in area V1 show a spatial attentional modulation. While the effect is small in magnitude, it is nevertheless highly significant. Interestingly, we see no effect in the subpopulation of the direction-selective neurons. We analysed our data in terms of the attentional mechanism. We did not find any support in our data for either of the attentional mechanisms. However, in particular the additive effect could have been obscured by a high level of noise.

With this result we could only partly support the hypothesis by Maunsell \& McAdams (2001), which suggested that a multiplicative attentional effect in lower area neurons is inherited by higher area neurons, resulting in a receptive field modulation. We do find an attentional effect, but this effect is a) not multiplicative and $b$ ) is only present in the non-direction selective subpopulation. As discussed earlier, particular the second point speaks against the hypothesis from Maunsell \& McAdams.

The second hypothesis from Womelsdorf et al. (2008) seems to account for our data better then the hypothesis by Maunsell \& McAdams. Womelsdorf proposed that while the attentional effect is inherited from lower area neurons, the multiplicative effect might be imposed onto the signal at the synaptic inputs of area MT itself. In order to be valid, our data does not need to show a multiplicative effect. Furthermore, as the model does not imply an inherited attentional effect from $\mathrm{V} 1$, there is no absolute necessity for our direction-selective subpopulation to show an attentional effect.

In light of our current results, the proposed hypothesis by Womelsdorf and colleagues does seem to account for our data and the likely explanation for the receptive field shifts in area MT. However, these results need to be verified by data from a different monkey. Furthermore, to reach a fuller understanding of the relationship of $\mathrm{V} 1$ and $\mathrm{MT}$ receptive fields and their interaction under different 
attentional states, a study of simultaneous recordings in these two areas with directly correlated receptive fields would be a logical step.

\section{Acknowledgments}

We thank Shubo Chakrabarti, Stephanie Westendorff and especially Cliodhna Quigley for valubale comments on the manuscript We thank Dirk Prüße, Leonore Burchardt and Sina Plümer for excellent technical assistance. 


\section{References}

Blasdel G, Campbell D (2001) Functional retinotopy of monkey visual cortex. Journal of Neuroscience 21:8286-8301.

Boynton GM (2011) Spikes, BOLD, Attention, and Awareness: A comparison of electrophysiological and fMRI signals in V1. Journal of Vision 11

Carrasco M, Ling S, Read S (2004) Attention alters appearance. Nature Neuroscience 7:308-313.

Chen Y, Seidemann E (2012) Attentional modulations related to spatial gating but not to allocation of limited resources in primate V1. Neuron 74: 557-566

Cohen MR, Maunsell JH (2009) Attention improves performance primarily

by reducing interneuronal correlations. Nature Neuroscience 12:1594 -1600.

Desimone R, Duncan J (1995) Neural mechanisms of selective visual attention. Annual Review of Neuroscience 18:193-222

Gattass R, Nascimento-Silva S, Soares JGM, Lima B, Jansen AK, Diogo ACM, Farias MF, Marcondes M, Botelho EP, Mariani OS, Azzi J, Fiorani M (2005) Cortical visual areas in monkeys: location, topography, connections, columns, plasticity and cortical dynamics. Philos. Trans. R. Soc. London Ser. B. 360, published online:709-731.

Gattass R, Sousa APB, Rosa MGP (1987) Visual topography of V1 in the cebus monkey. Journal of Comparative Neurology 259: 529-548

Gur M, Snodderly DM (2007) Direction selectivity in V1 of alert monkeys: evidence for parallel pathways for motion processing. Journal of Physiology 585(2): 383-400 Hubel DH, Wiesel TN (1974) Uniformity of the monkey striate cortex: a parallel relationship between field size, scatter and magnification factor. Journal of Comparative Neurology 158: 295-306

Liu J, Newsome WT (2003) Functional organization of speed tuned neurons in visual area MT. Journal of Neurophysiology 89: 246-256

Livingstone MS (1998) Mechanisms of direction selectivity in macaque V1. Neuron 20: 509-526

Luck SJ, Chelazzi L, Hillyard SA, Desimone R (1997) Neural mechanisms of spatial selective attention in areas $\mathrm{V} 1, \mathrm{~V} 2$, and $\mathrm{V} 4$ of macaque visual cortex. Journal of Neurophysiology 77:24-42.

Maunsell JHR, Cook EP (2002) The role of attention in visual processing. Philos. Trans. R. Soc. London Ser. B 357:1063-1072 
Maunsell JHR, McAdams CJ (2001) Effects of attention on the responsiveness and selectivity of individual neurons in visual cerebral cortex. In: Visual attention and cortical circuits(Braun, J. et al., eds), pp 103-120 Cambridge, Massachusetts: MIT Press.

Maunsell JHR, Treue S (2006) Feature-based attention in visual cortex. Trends in Neuroscience 29:317-322.

McAdams CJ, Maunsell JHR (1999) Effects of attention on orientation-tuning functions of single neurons in Macaque cortical area V4. Journal of Neuroscience 19:431-441.

McAdams C, Reid RC (2003) Effects on attention on the spatial and temporal structure of receptive fields in macaque primary visual cortex. Society for Neuroscience, Annual Meeting .

Motter BC (1993) Focal attention produces spatially selective processing in visual cortical areas V1, V2, and V4 in the presence of competing stimuli. Journal of Neurophysiology 70:909-919.

Movshon JA, Newsome WT (1996) Visual response properties of striate cortical neurons projecting to area MT in macaque monkeys. Journal of Neuroscience 16:7733-7741.

Müller NG. Bartelt OA, Donner TH, Villringer A, Brandt SA (2003). Physiological correlate of the 'zoom lens' of visual attention. Journal of Neuroscience 23(9): 3561-3565.

Olshausen B, Field D (2005) How close are we to understanding V1? Neural Computation 17: 1665-1699

Pooresmaeili A, Poort J, Thiele A, Roelfsema PR (2010) Separable codes for attention and luminance contrast in the primary visual cortex. Journal of Neuroscience 30:12701-12711.

Posner MI (1980) Orienting of attention. Quarterly Journal of Experimental Psychology 32:3-25.

Snowden RJ, Treue S, Andersen RA (1992) The response of neurons in areas V1 and MT of the alert rhesus monkey to moving random dot patterns. Experimental Brain Research 88:389-400.

Super H, Roelfsema PR (2005) Chronic multiunit recordings in behaving animals: advantages and limitations. Progress in Brain Research 147:263-282. 
Thiele A, Pooresmaeili A, Delicato LS, Herrero JL, Roelfsema PR (2009) Additive Effects of Attention and Stimulus Contrast in Primary Visual Cortex. Cerebral Cortex 19:2970-2981.

Womelsdorf T, Fries P, Mitra PP, Desimone R (2006) Gamma-band synchronization in visual cortex predicts speed of change detection. Nature 439:733-736.

Womelsdorf T, Anton-Erxleben K, Treue S (2008) Receptive field shift and shrinkage in macaque middle temporal area through attentional gain modulation. Journal of Neuroscience 28:8934-8944. 
Supplementary Materials for

\section{Spatial attentional modulation of multi-unit activity in macaque $\mathrm{V} 1$}

Valeska M. Stephan, Rui-Feng Lui \& Stefan Treue 


\section{Multielectrode array and neuronal signals}

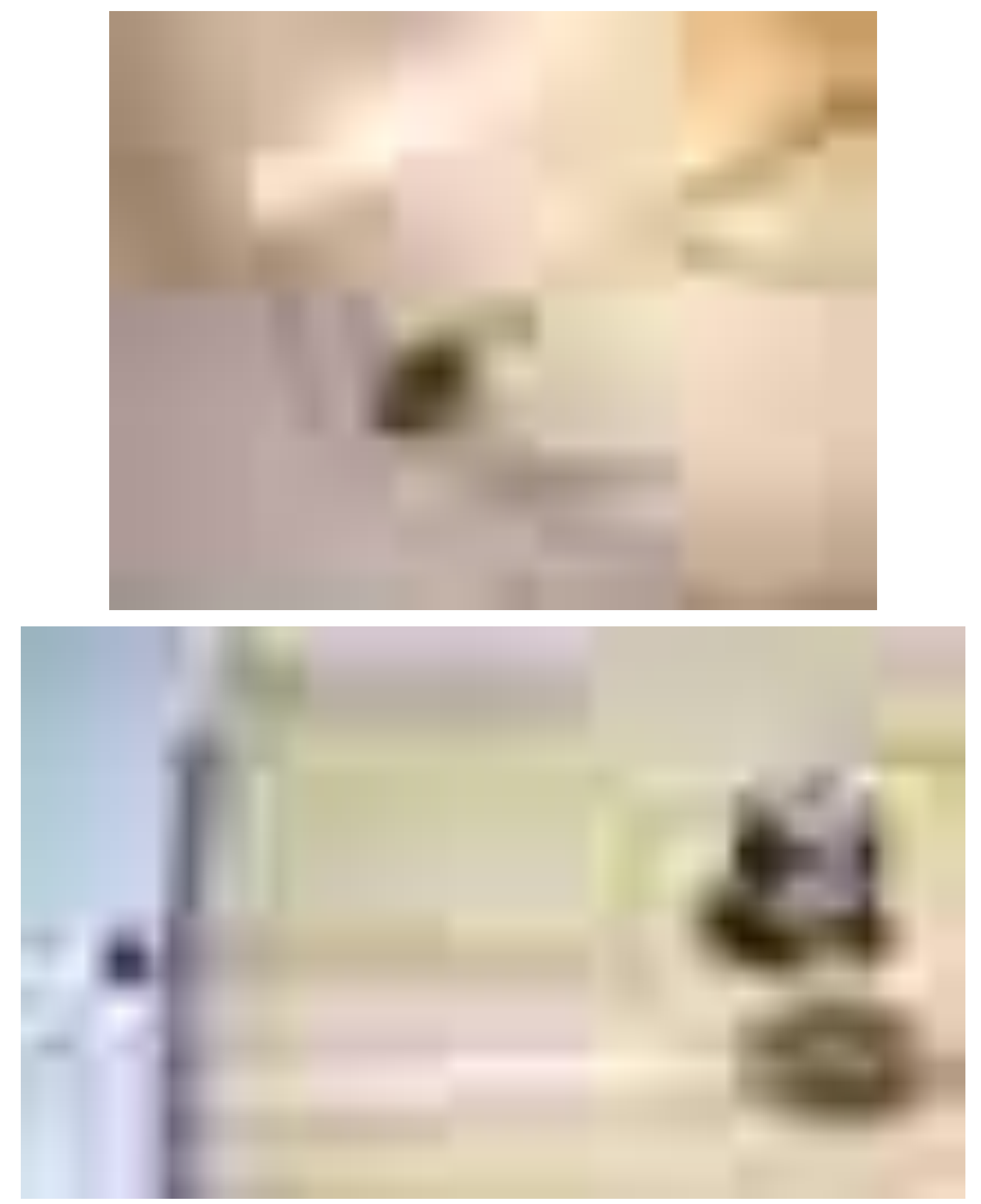

Figure S1 - Multielectrode CerePort Utah Array (BlackRock)

Upper - View on the array from the side (match as size comparison). The array consists of $10 \times 10$ electrodes and is $5 \times 5 \mathrm{~mm}$ in size. The depth of each electrode is $1.5 \mathrm{~mm}$ and the inter-electrode distance is 400 micrometre. A bundle of wires connect the electrodes with the connector (lower). The connector is positioned on the monkeys skull and serves as a connection to the Omniplex recording system. Two additional grounding wires are attached to the connector. 

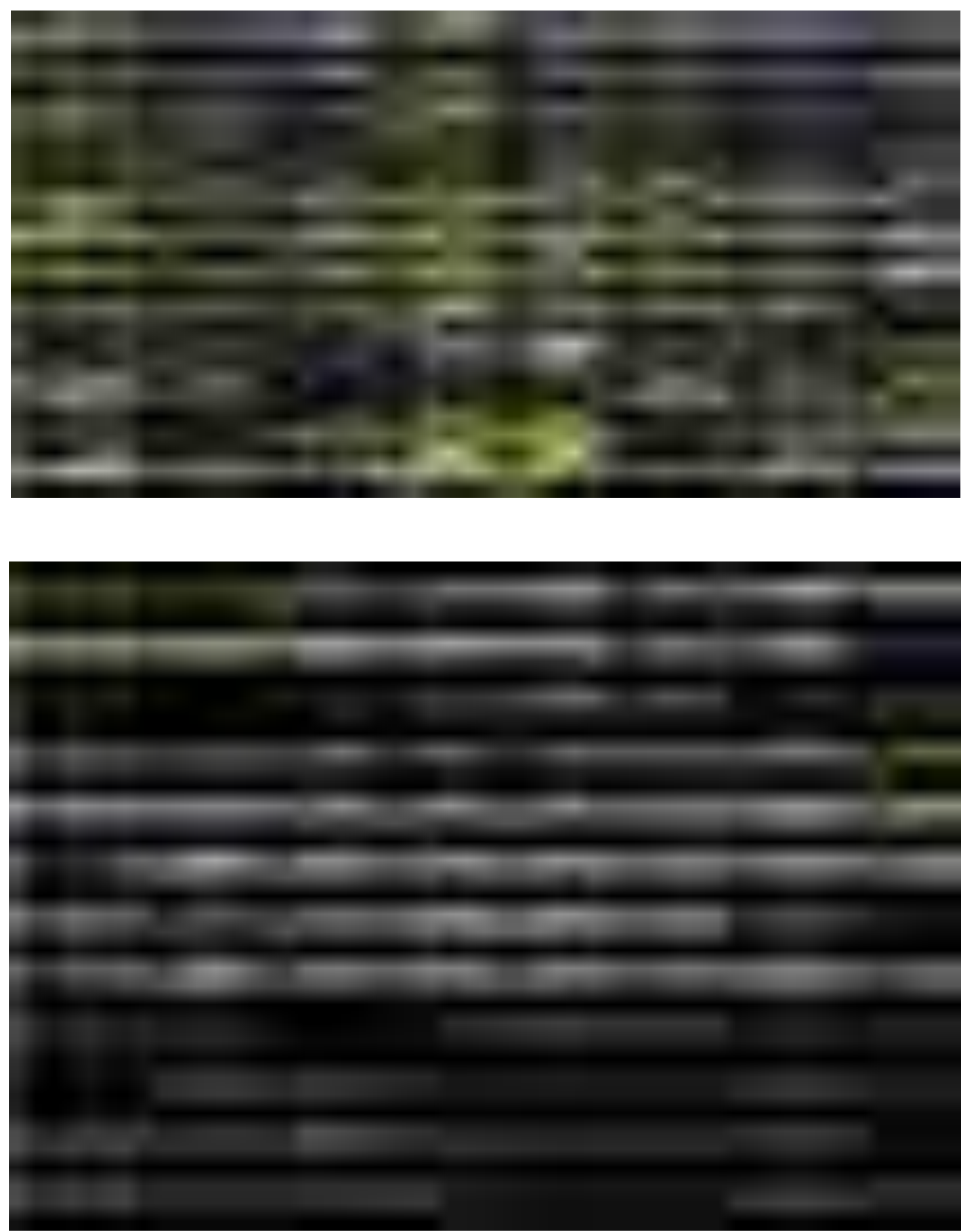

Figure S2 - Waveforms, (one week) after implantation and (eight weeks) after implantation (lower) Each square window represents the signals of one electrode. Isolated single units are represented in either yellow or green. Signals, which could not be isolated manually, are represented in grey. We observed a rapid decline in signal quality over the first two months. Isolation of single units was soon not possible anymore. Therefore we conducted our analysis using multiunit activity. 


\section{Analysis of receptive fields and verification of array position in the cortex}

To verify the position of the array, we correlated the centroids position with the actual distance to the fovea on the cortex (Fig S3 \& S4). Furthermore we validated the centroids positions to the horizontal meridian (Fig S5 \& S6).

We first calculated the position of the centroid for the receptive field of each electrode (Fig. S4, S5). The average value of the centroids was computed and the position was defined at the center of the array. The position was topographically correlated to the actual cortex surface (Gattass et al., 1987). The actual positions on the cortex were described with polar coordinates, tempolateral points. The horizontal meridian of $\mathrm{V} 1$ was defined as pole and polar axis respectively (Gattass et al., 1987). All 96 electrode positions on the cortex were calculated relative to position of the array centre. The receptive fields in the monkeys visual field were plotted from neuronal signals as a function of the actual positions of the responding electrodes in the cortex (S6-S9). The results are consistent with the previous studies that showed topographical organization in the primary cortex (e.g. Gattass et al. 1987; Blasdel and Campbell, 2001). 
Figure S3 - Distribution of recordingsites in the array.

View onto the array from the top. Each number refers to one recordingsite. Colorcoding was done as reference to correlate centroids to recordingsite. The array was implanted into the cortex so that the top (Wire(Frontal)) faced anterior, right side of the array faced medial, left side faced lateral and down side faced posterior. 


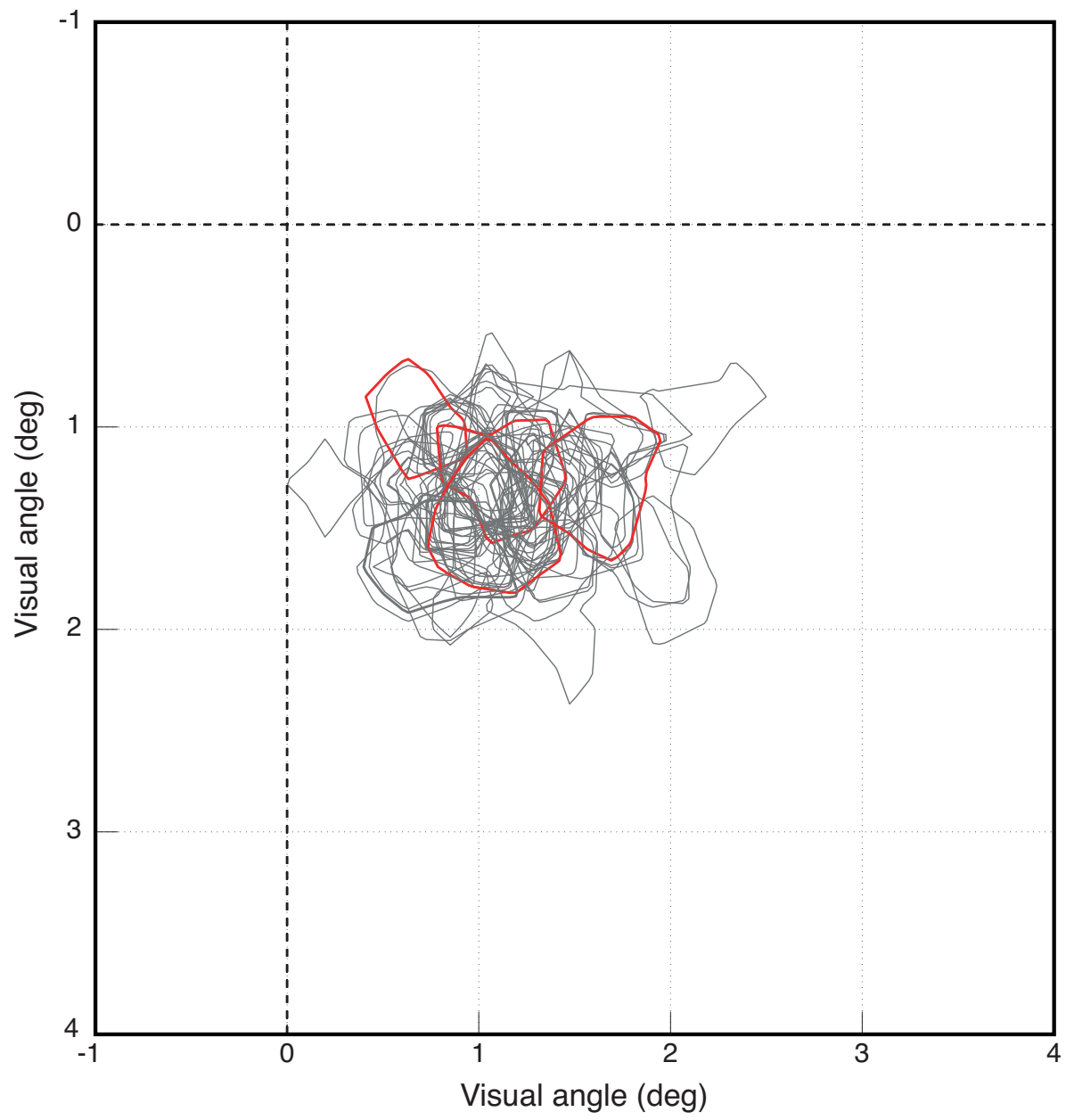

Figure S4 - Receptive Field outlines

Every grey line marks the outline of one receptive field (four example receptive fields are indicated with red lines). Receptive field outlines are defined as the half maximum response (see M\&M). $x / y$ are axis in visual degree. The position of the fovea is at 0/0 (cross of the dotted lines) 


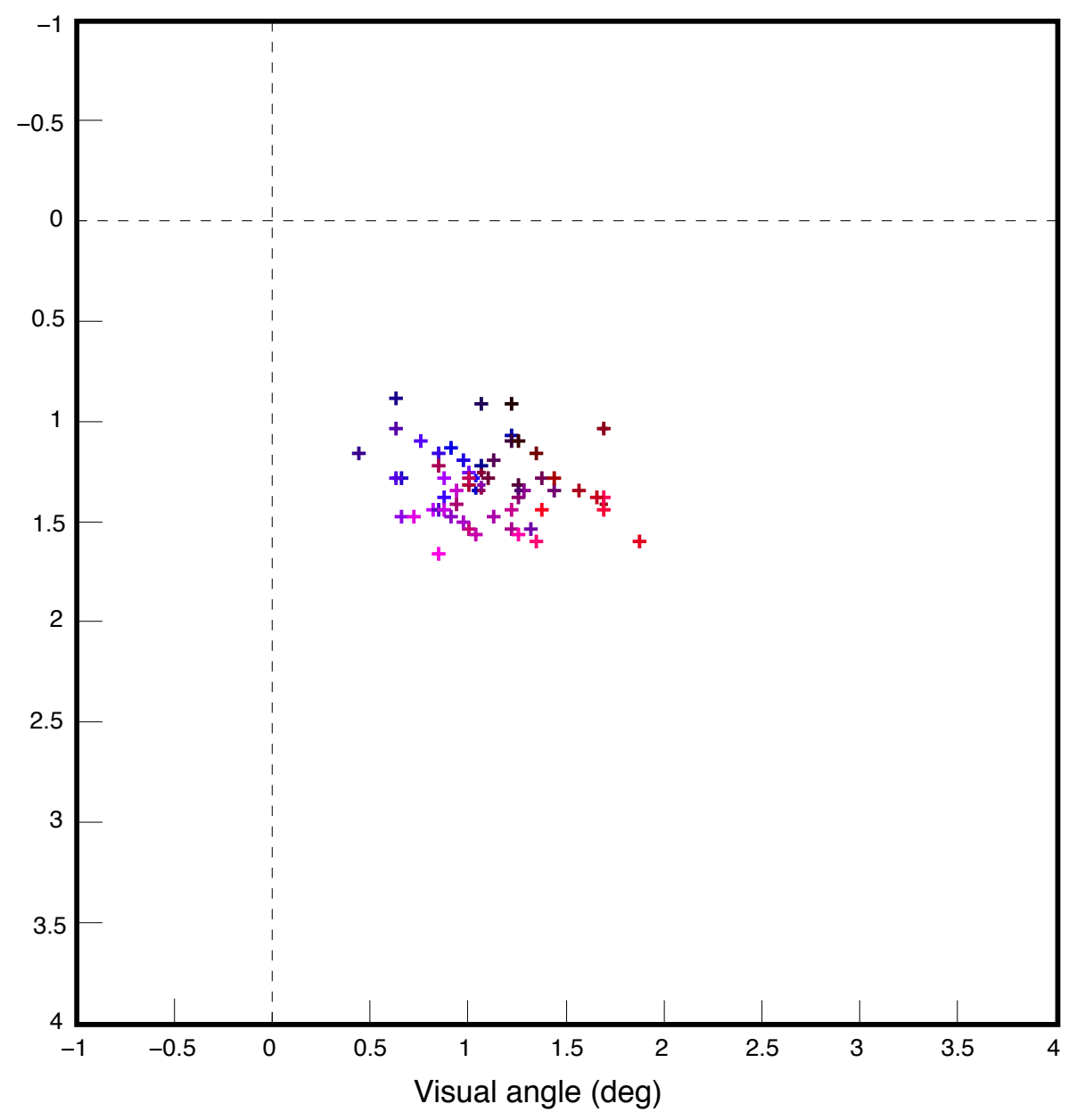

Figure S5- Position of receptive field centroids.

Every cross marks the centroid of one receptive field. The colour code represents the position of the electrodes in the array (fig. S 5). The $x / y$-axis depict visual degree. Position of the fovea is $0 / 0$ visual degree (cross of the dotted lines). 


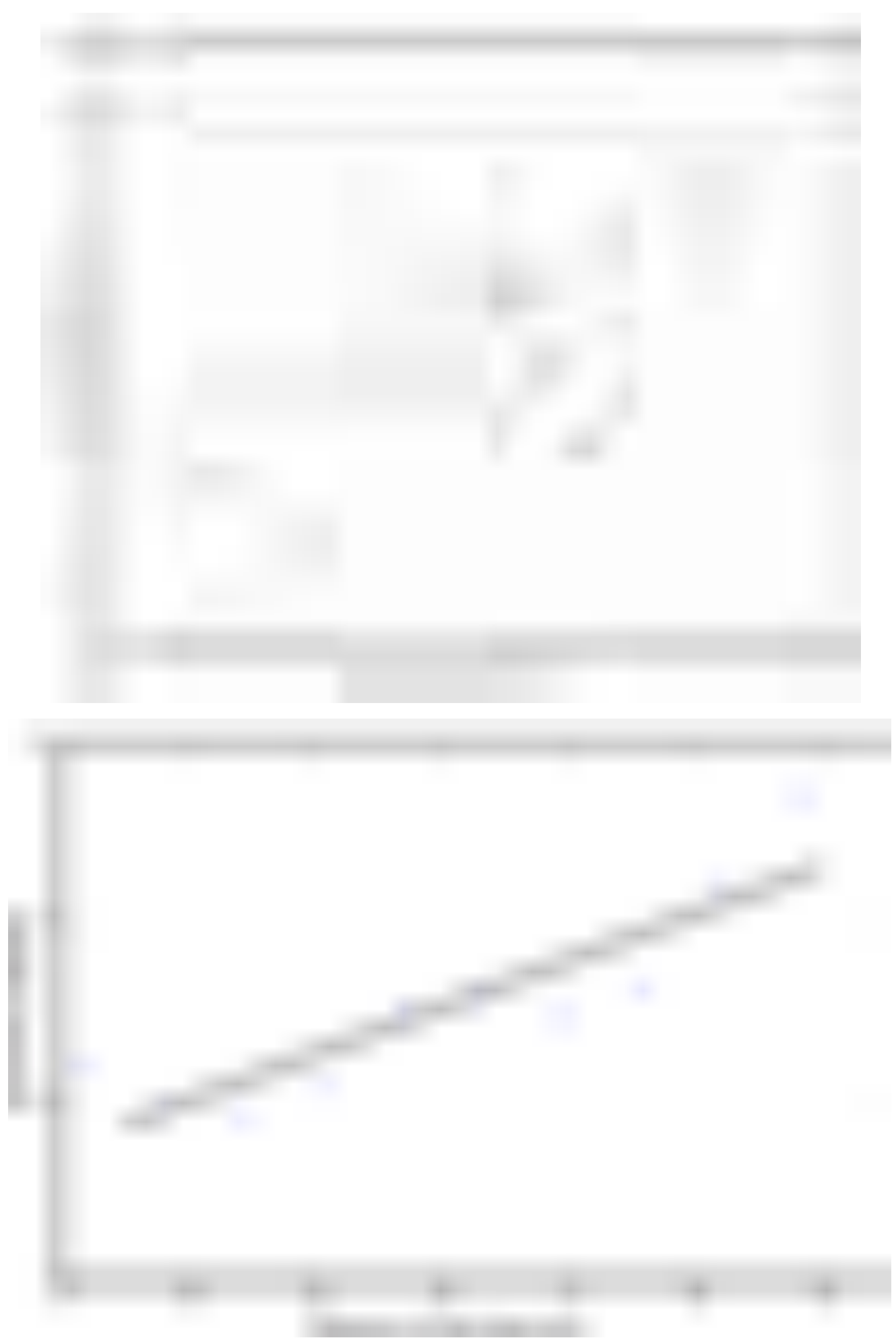

Figures S6 \& S7 - The eccentricity of receptive fields as a function of distance of the electrodes to original fovea on cortex. The asterisks in the section denote the receptive filed centroids in animal's visual field and electrodes positions in cortex. The numbers beside the asterisks indicate the electrodes in the array (Fig. S3). All plotted data points were binned into 10 equally spaced containers, the vertical broken lines showed the container edges. The average position of receptive fields in each group was calculated and plotted in S6. 


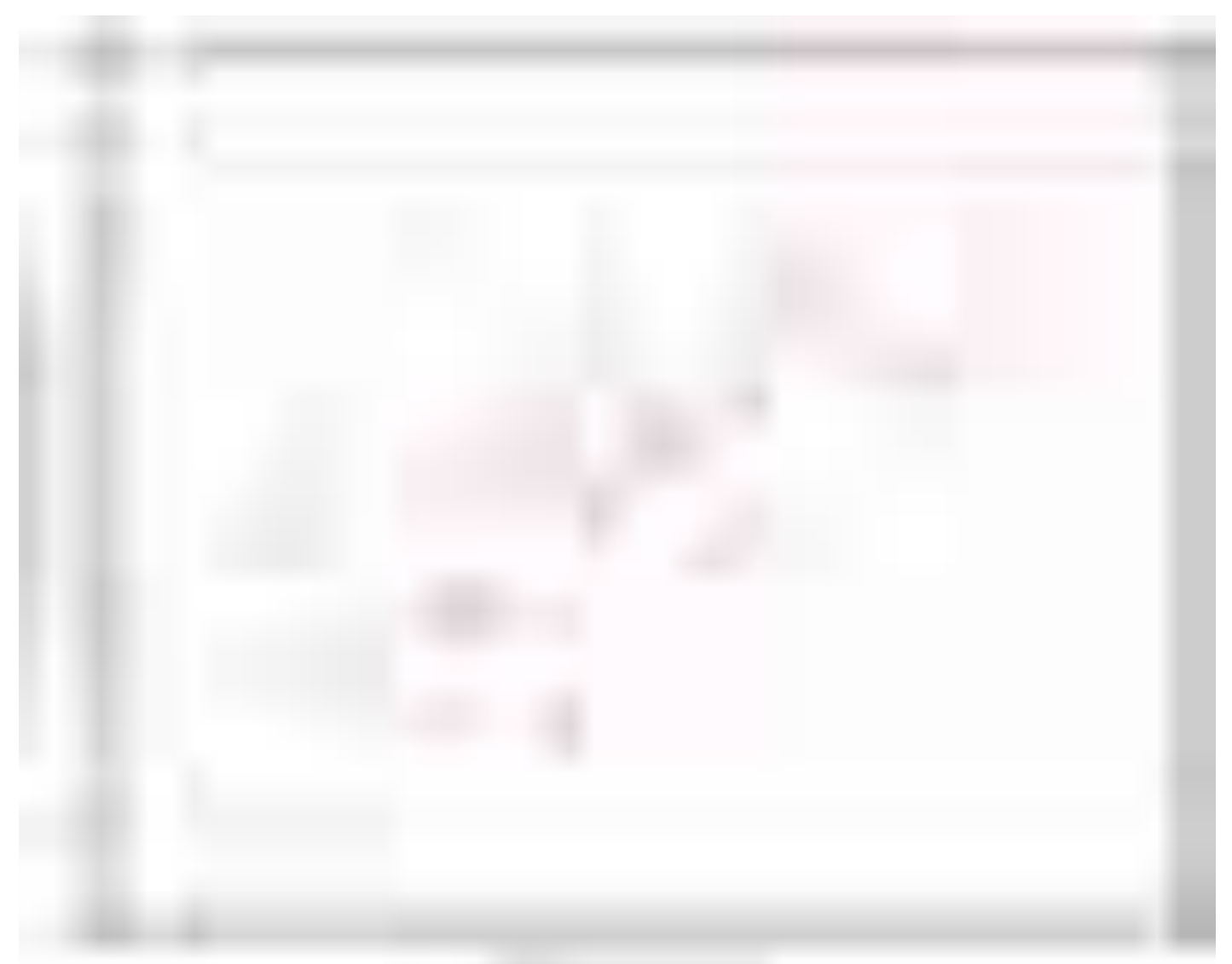

Figures $\mathbf{S 8 ~ \& ~} \mathbf{S 9}$ - The distance from centroids of receptive fields to horizontal median in visual field was monotonically increased when electrodes positions moved away from horizontal median of primary cortex. Labeling conventions as in S6 \& S7 respectively. 


\section{Stimulus positions ("with probe" condition)}

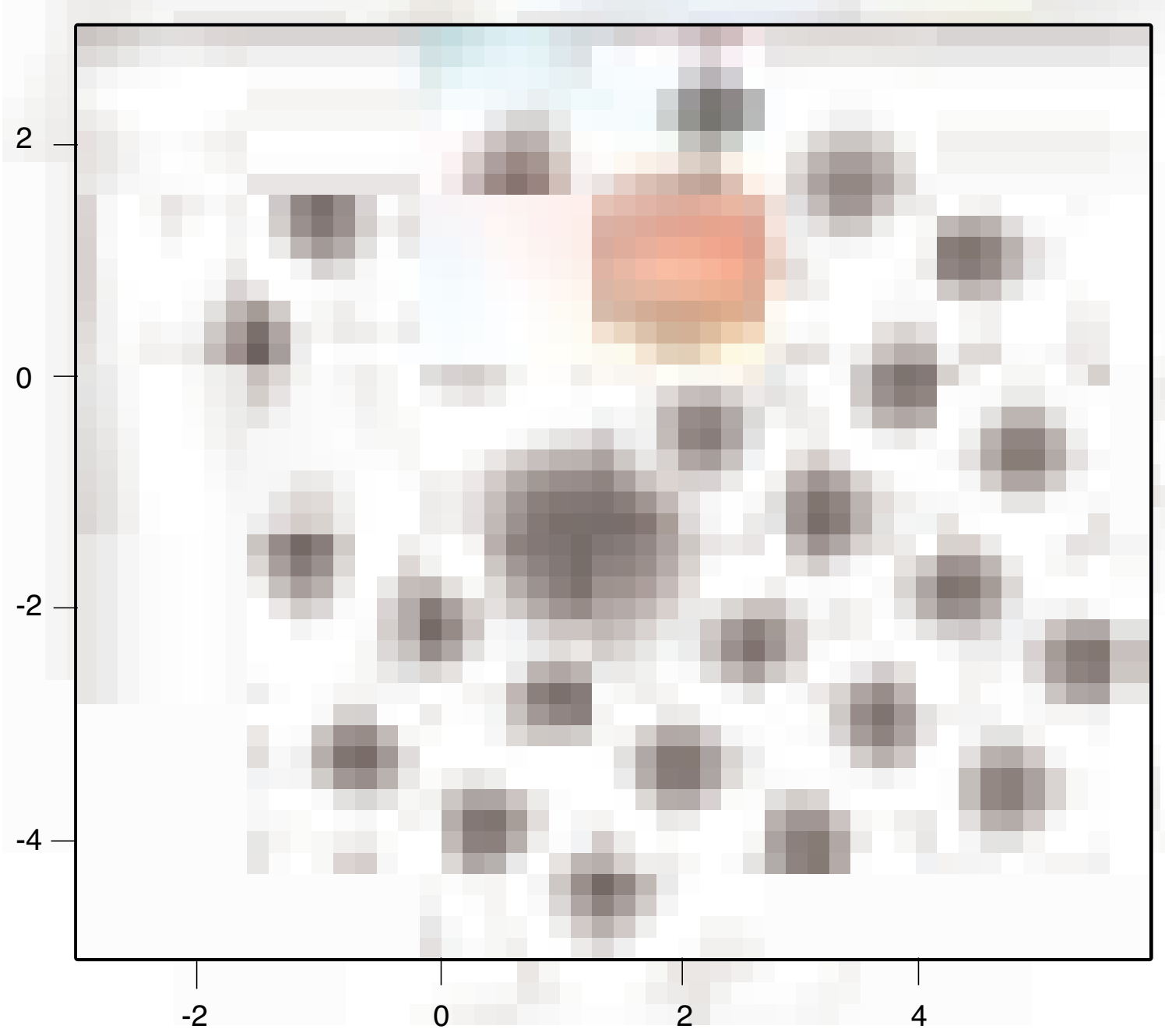

Figure S10 - Stimulus arrangements and receptive fields

$\mathrm{X} / \mathrm{Y}$ axis are in visual degree. Circles indicate the stimuli positions in the visual field (red - outside stimulus; brown -inside stimulus). Grey lines indicate receptive field size and positions; in red four receptive fields are highlighted as random examples (see figure S7). The black dashed square indicates size and position of fixation point. Small dots at the fixation point indicate average eyepositions for different attentional conditions (red - attention outside, brown - attention inside, yellow - fixation only). Dark grey medium sized dots indicate positions of the probe stimuli ("with probe" condition).

\section{References}

Gattass R, Sousa APB, Rosa MGP (1987) Visual topography of V1 in the cebus monkey. Journal of Comparative Neurology 259: 529-548

Blasdel G, Campbell D (2001) Functional retinotopy of monkey visual cortex. Journal of Neuroscience 21:8286-8301 



\section{Summary}

This thesis examined the influences of attention on the processing of complex and simple motion stimuli.

The work constited of two physiology studies. In the first study we investigated the question of how complex motion stimuli, namely transparent motion stimuli, are processed in area MT and the influence of attention on the processing.

We recorded single-unit activity in the area MT of two behaving macaque monkeys. The animals were instructed to attend to a fixation point, to a moving transparent random dot pattern inside the receptive field or to a moving transparent random dot pattern outside the receptive field. The direction of motion of the random dot pattern was systematically varied while keeping a constant angle between the two direction vectors. We obtained tuning curves for the three attentional conditions.

The population activity profiles show a bilobed profile. This corresponds to those stimulus configurations in which one of the direction components moved in the neurons preferred direction. We found an attentional modulation of the bilobed tuning profile. When attention was shifted from the fixation point to the stimulus inside the receptive field we observed an upregulation of the bilobed tuning profile. Furthermore, we observed an upregulation of the same magnitude when attention was allocated on the stimulus outside the receptive field. These findings -an absence of spatial attention as well as the absence of the suppressive effect by non-preferred features in the feature-based condition- might be explained by an behavioural strategy of the animals to solve the demanding task or that area MT is not the target area for separating the motion signals of overlaying surfaces as it is the case for transparent motion stimuli. Further investigations have to be conducted to clarify these remaining questions.

In our second study we were interested in different aspect of attentional effects on visual motion stimuli. It was found that spatial attention in area MT influences the spatial selectivity of neurons, by modulating the receptive field (Womelsdorf et al., 2006). When attenting to one of two motion stimuli within the receptive field of a neuron, the spatial selectivity will shift towards the spatial focus of attention. This receptive field modulation represents a non-multiplicative modulation. This effect is 
unusual, as effects of spatial attention have mostly been described to be multiplicative. Two hypothesis might account for this interesting finding. Maunsell \& McAdams (2001) suggested that an effect on spatial selectivity is inherited from lower area neurons. The response of the lower area neurons might be multiplicatively modulated, which would lead to a receptive field shift in higher area neurons. Womelsdorf et al. (2008) proposed a different approach to the problem. They suggested that while the attentional modulation, which leads to receptive field shift, is multiplicative, the effect might not be inherited from lower visual areas, but being a result of a weighing of the synaptic inputs of neurons in area MT. To investigate which of those two hypothesis accounts for the observed receptive field shifts, we recorded from visual area $\mathrm{V} 1$, which projects directly to area MT, while the monkey did a spatial attention task. We found an attentional modulation of $3-4 \%$ for the whole population of neurons we recorded from. We did not however find an attentional effect for a subpopulation of direction selective cells. This is a suprising effect, because if the first hypothesis holds true, we should find an attentional effect especially in the direction selective cells, which project from area V1 to MT. Furthermore, we do not find that our attentional effect is multiplicative. We conclude therefore that our data supports the second hypothesis and rather then inheriting the attentional effect from lower area neurons, receptive field shifts in area MT come to pass due to a modulation at the cells synaptic inputs.

This thesis dealt with the attentional effects on different motion stimuli. We found that primarily feature based effects contribute to the processing of transparent motion in area MT. Furthermore we showed that the effect of spatial attention on the spatial selectivity of neurons in the visual system is likely to be contributed to a local effect on the inputs of neurons in area MT. 


\section{Bibliography}

Albright TD, Desimone R, Gross CG (1984) Columnar organization of directionally selective cells in visual area MT of the macaque. Journal of Neurophysiology 51(1): 16-31

Adams DL, Horton JC (2003) A precise retinotopic map of primate striate cortex generated from the representation of angioscotomas. Journal of Neuroscience 23:3771-3789.

Adelson EH, Bergen JR (1985) Spatiotemporal energy models for the perception of motion. JOSA A 2:284-299.

Albright TD, Desimone R, Gross CG (1984) Columnar organization of directionally selective cells in visual area MT of the macaque. Journal of Neurophysiology 51:16-31.

Alonso J-M, Chen Y (2008) Receptive fields. Scholarpedia, 4(1):5393.

Andersen RA, Snowden RJ, Treue S, Graziano M (1990) Hierarchical processing of motion in the visual cortex of monkey. Cold Spring Harb Symp Quant Biol 55:741-748.

Angelucci A, Levitt JB, Walton EJS, Hupe JM, Bullier J, Lund JS (2002) Circuits for local and global signal integration in primary visual cortex. Journal of Neuroscience 22:8633-8646.

Anton-Erxleben K, Henrich C, Treue S (2007) Attention changes perceived size of moving visual patterns. Journal of Vision 7:5, 1-9.

Anton-Erxleben K, Stephan VM, Treue S (2009) Attention reshapes centersurround receptive field structure in macaque cortical area MT. Cerebral Cortex 19:2466-2478.

Bisley JW (2011) The neural basis of visual attention. Journal of Physiology 589:49-57.

Blasdel G, Campbell D (2001) Functional retinotopy of monkey visual cortex. Journal of Neuroscience 21:8286-8301.

Berens P, Ecker AS, Cotton RJ, Ma WJ, Bethge M, Tolias AS (2012) A fast and simple population code for orientation in primate V1. Journal of Neuroscience 32:10618-10626

Born RT, Bradley DC (2005) Structure and function of visual area MT. Annual Review of Neuroscience 28:157-189. 
Boynton GM (2011) Spikes, BOLD, Attention, and Awareness: A comparison of electrophysiological and fMRI signals in V1. Journal of Vision 11.

Braddick O, Qian N (2001) The organization of global motion and transparency. In: Motion Vision - Computational, Neural, and Ecological Constraints(Zanker, J. M. and Zeil, J., eds), pp 85-112 Berlin Heidelberg New York: Spinger Verlag.

Bradley DC, Andersen RA (1998) Center-surround antagonism based on disparity in primate area MT. Journal of Neuroscience 18:7552-7565.

Buracas GT, Boynton GM (2007) The effect of spatial attention on contrast response functions in human visual cortex. Journal of Neuroscience 27:93-97.

Busse L, Wade AR, Carandini M (2009) Representation of concurrent stimuli by population activity in visual cortex. Neuron 64:931-942.

Callaway EM (1998) Local circuits in primary visual cortex of the macaque monkey. Annual Review of Neuroscience 21:47-74.

Carandini M, Heeger DJ (2012) Normalization as a canonical neural computation. Nature Reviews Neuroscience 13: 51-62

Carandini, M (2012) Area V1. Scholarpedia, 7(7):12105.

Carandini M, Heeger DJ, Senn W (2002) A synaptic explanation of suppression in visual cortex. 1-23.

Carandini M, Heeger DJ, Movshon JA (1996) Linearity and gain control in V1 simple cells. In: Cerebral Cortex, Vol. 13: Cortical Models (Jones, E.G. and Ulinski, P.S. eds), New York: Plenum Press.

Carrasco M (2011) Visual attention: The past 25 years. Vision Research 51:14841525.

Carrasco M, Ling S, Read S (2004) Attention alters appearance. Nature Neuroscience 7:308-313.

Cavanaugh JR, Bair W, Movshon JA (2002) Selectivity and spatial distribution of signals from the receptive field surround in macaque $V 1$ neurons. Journal of Neurophysiology 88:2547-2556.

Chelazzi L, Miller EK, Duncan J, Desimone R (2001) Responses of Neurons in Macaque area V4 during memory-guided visual search. Cerebral Cortex 11:761772.

Chen Y, Seidemann E (2012) Attentional modulations related to spatial gating but not to allocation of limited resources in primate V1. Neuron 74: 557-566 
Chen Y, Martinez-Conde S, Macknik SL, Bereshpolova Y, Swadlow HA, Alonso JM (2008) Task difficulty modulates the activity of specific neuronal populations in primary visual cortex. Nature Neuroscience 11:974-982.

Churchland MM, Priebe NJ, Lisberger SG (2005) Comparison of the spatial limits on direction selectivity in visual areas MT and V1. Journal of Neurophysiology 93:1235-1245.

Connor CE, Gallant JL, Preddie DC, Van Essen DC (1996) Responses in area V4 depend on the spatial relationship between stimulus and attention. Journal of Neurophysiology 75:1306-1309.

Connor CE, Preddie DC, Gallant JL, Van Essen DC (1997) Spatial attention effects in macaque area V4. Journal of Neuroscience 17:3201-3214.

Croner LJ, Albright TD (1999) Segmentation by color influences responses of motion-sensitive neurons in the cortical middle temporal visual area. Journal of Neuroscience 19:3935-3951.

Cumming BG, DeAngelis GC (2001) The physiology of stereopsis. Annual Review of Neuroscience 24:203-238.

Daniel PM, Whitteridge D (1961) The representation of the visual field on the cerebral cortex in monkeys. Journal of Physiology (London) 159: 203-221

DeAngelis GC, Newsome WT (1999) Organization of disparity-selective neurons in macaque area MT. Journal of Neuroscience 19:1398-1415.

Desimone R, Duncan J (1995) Neural mechanisms of selective visual attention. Annual Review of Neuroscience 18:193-222.

Felleman DJ, Van Essen DC (1991) Distributed hierarchical processing in the primate cerebral cortex. Cerebral Cortex 1:1-47.

Ferster D (1986) Orientation selectivity of synaptic potentials in neurones of Cat primary visual cortex. Journal of Neuroscience 6:1284-1301.

Gattass R, Nascimento-Silva S, Soares JGM, Lima B, Jansen AK, Diogo ACM, Farias MF, Marcondes M, Botelho EP, Mariani OS, Azzi J, Fiorani M (2005) Cortical visual areas in monkeys: location, topography, connections, columns, plasticity and cortical dynamics. Philos. Trans. R. Soc. London Ser. B. 360, published online:709-731.

Gattass R, Sousa APB, Rosa MGP (1987) Visual topography of V1 in the cebus monkey. Journal of Comparative Neurology 259: 529-548 
Gattass R, Gross CG. 1981. Visual Topography of Striate Projection Zone (Mt) in Posterior Temporal Sulcus of the Macaque. Journal of Neurophysiology 46: 62138

Gegenfurtner KR, Kiper DC (2003) Color Vision. Annual Review of Neuroscience 26:181-206.

Ghose GM, Maunsell JH (2008) Spatial summation can explain the attentional modulation of neuronal responses to multiple stimuli in area V4. Journal of Neuroscience 28:5115-5126.

Groh JM, Born RT, Newsome WT (1997) How is the sensory map read out ? Effects of microstimulation in visual area MT on saccades and smooth pursuit eye movements. Journal of Neuroscience 17:4312-4330.

Gur M, Snodderly DM (2007) Direction selectivity in V1 of alert monkeys: evidence for parallel pathways for motion processing. Journal of Physiology 585(2): 383-400 Haenny PE, Schiller PH (1988). State dependent activity in monkey visual cortex. I. Single cell activity in V1 and V4 on visual tasks. Exp. Brain Res. 69, 225-244 Heuer HW, Britten KH (2002) Contrast dependence of response normalization in area MT of the rhesus macaque. Journal of Neurophysiology 88:3398-3408.

Hubel DH, Wiesel TN. Receptive fields of single neurones in the cat's striate cortex. Journal of Physiology 148:574-91, 1959

Hubel DH (1963) The visual cortex of the brain. Scientific American 209: 54-63

Hubel DH, Wiesel TN (1977) Ferrier lecture. Functional architecture of macaque monkey visual cortex. Proc R Soc Lond B Biol Sci 198:1-59

Hubel DH, Wiesel TN (1974a) Sequence regularity and geometry of orientation columns in the monkey striate cortex. Journal of Comparative Neurology 158: 267294

Hubel DH, Wiesel TN (1974b) Uniformity of the monkey striate cortex: a parallel relationship between field size, scatterand magnification factor. Journal of Comparative Neurology 158: 295-306

Katzner S, Busse L, Treue S (2006) Feature-based attentional integration of color and visual motion. Journal of Vision 6:7: 269-284.

Kozyrev V, Lochte A, Daliri R, Treue $S$ (prepared for submission) Attentional modulation of population responses in macaque area MT to the direction of spatially seperated motion patterns 
Knudsen El (2007) Fundamental components of attention. Annual Review of Neuroscience 30:57-78.

Lankheet MJM, Verstraten FAJ (1995) Attentional modulation of adaptation to two-component transparent motion. Vision Research 35:1401-1412.

Lee J, Maunsell JH (2009) A normalization model of attentional modulation of single unit responses. PLoS One 4:e4651.

Lee J, Maunsell JH (2010) Attentional modulation of MT neurons with single or multiple stimuli in their receptive fields. Journal of Neuroscience 30:3058-3066.

Livingstone MS, Hubel DH (1984) Anatomy and physiology of a color system in the primate visual cortex. Journal of Neuroscience 4:309-356.

Livingstone MS (1998) Mechanisms of direction selectivity in macaque V1. Neuron 20: 509-526

Livingstone MS, Pack CP, Born RT (2001) Two-dimensional substructure of MT receptive fields. Neuron 30(3):781-793.

Liu J, Newsome WT (2003) Functional organization of speed tuned neurons in visual area MT. Journal of Neurophysiology 89: 246-256

Luck SJ, Chelazzi L, Hillyard SA, Desimone R (1997) Neural mechanisms of spatial selective attention in areas $\mathrm{V} 1, \mathrm{~V} 2$, and $\mathrm{V} 4$ of macaque visual cortex. Journal of Neurophysiology 77:24-42.

Majaj NJ, Carandini M, Movshon JA (2007) Motion integration by neurons in macaque MT is local, not global. Journal of Neuroscience 27:366-370.

Marcus DS, Van Essen DC (2002) Scene segmentation and attention in primate cortical areas V1 and V2. Journal of Neurophysiology 88:2648-2658.

Martinez-Trujillo JC, Treue S (2002) Attentional modulation strength in cortical area MT depends on stimulus contrast. Neuron 35:365-370.

Martinez-Trujillo JC, Treue S (2004) Feature-based attention increases the selectivity of population responses in primate visual cortex. Current Biology 14:744-751.

Maunsell JHR, Cook EP (2002) The role of attention in visual processing. Philos. Trans. R. Soc. London Ser. B 357:1063-1072.

Maunsell JHR, McAdams CJ (2001) Effects of attention on the responsiveness and selectivity of individual neurons in visual cerebral cortex. In: Visual attention and cortical circuits(Braun, J. et al., eds), pp 103-120 Cambridge, Massachusetts: MIT Press. 
Maunsell JHR, Newsome WT (1987) Visual processing in monkey extrastriate cortex. Annual Review of Neuroscience 10:363-401.

Maunsell JHR, Treue S (2006) Feature-based attention in visual cortex. Trends in Neuroscience 29:317-322.

McAdams CJ, Maunsell JHR (1999) Effects of attention on orientation-tuning functions of single neurons in Macaque cortical area V4. Journal of Neuroscience 19:431-441.

McAdams C, Reid RC (2003) Effects on attention on the spatial and temporal structure of receptive fields in macaque primary visual cortex. Society for Neuroscience, Annual Meeting .

McAlonan K, Cavanaugh J, Wurtz RH (2008) Guarding the gateway to cortex with attention in visual thalamus. Nature 456:391-394.

Montijn JS, Klink PC, vanWezel RJA (2012) Divisive normalization and neuronal oscillations in a single hierachical framework of selective visual attention. Frontiers in Neural Circuits 6: 1-17

Moran J, Desimone R (1985) Selective attention gates visual processing in the extrastriate cortex. Science 229:782-784.

Motter BC (1993) Focal attention produces spatially selective processing in visual cortical areas V1, V2, and V4 in the presence of competing stimuli. Journal of Neurophysiology 70:909-919.

Movshon JA, Thompson ID, Tolhurst DJ. 1978. Spatial and Temporal Contrast Sensitivity of Neurons in Area 17 and 18 of the Cat's Visual Cortex. Journal of Physiology 283: 101-20

Movshon JA, Adelson EH, Gizzi MS, Newsome WT (1985) The analysis of moving visual patterns. In Chagas C, Gattass R, Gross C (eds.) Pattern recognition mechanisms - Pontificiae Academiae Scientiarum Scripta Varia 54. Heidelberg, Springer-Verlag: 117-151.

Movshon JA, Newsome WT (1996) Visual response properties of striate cortical neurons projecting to area MT in macaque monkeys. Journal of Neuroscience 16:7733-7741.

Müller NG. Bartelt OA, Donner TH, Villringer A, Brandt SA (2003). Physiological correlate of the 'zoom lens' of visual attention. Journal of Neuroscience 23(9): $3561-3565$. 
Murray SO (2008) The effects of spatial attention in early human visual cortex are stimulus independent. Journal of Vision 3:article 2.

Nassi JJ, Lyon DC, Callaway EM (2006) The parvocellular LGN provides a robust disynaptic input to the visual motion area MT. Neuron 50(2): 319-327

Newsome WT, Britten KH, Movshon JA, Shadlen M (1989) Single neurons and the perception of visual motion. In: Neural Mechanisms of Visual Perception, Proceedings of the Retina Research Foundation Symposia Volume Two(Lam, D. M.-K. and Gilbert, C. D., eds), pp 171-198 Houston, TX: Gulf Publishing Company. O'Craven KM, Rosen BR, Kwong KK, Treisman A, Savoy RL (1997) Voluntary attention modulates fMRI activity in human MT-MST. Neuron 18:591-598.

Olshausen B, Field D (2005) How close are we to understanding V1? Neural Computation 17: 1665-1699

Orban GA, Kennedy H, Bullier J. (1986). Velocity Sensitivity and Direction Selectivity of Neurons in Area V1 and V2 of the Monkey: Influence of Eccentricity. Journal of Neurophysiology 56: 462-80

Orban GA, Van Essen DC, Vanduffel W. (2004). Comparative Mapping of Higher Visual Areas in the Monkeys and Humans. Trends in cognitive Neuroscience 8: 315-24

Patzwahl D, Treue S (2009) Combining spatial and feature-based attention within the receptive field of MT neurons. Vision Research 49:1188-1193.

Pooresmaeili A, Poort J, Thiele A, Roelfsema PR (2010) Separable codes for attention and luminance contrast in the primary visual cortex. Journal of Neuroscience 30:12701-12711.

Posner MI (1980) Orienting of attention. Quarterly Journal of Experimental Psychology 32:3-25.

Posner MI, Gilbert CD (1999) Attention and primary visual cortex. PNAS 96:25852587.

Prince SJD, Pointon AD, Cumming BG, Parker AJ (2000) The precision of single neuron responses in cortical area $\mathrm{V} 1$ during stereoscopic depth judgements. Journal of Neuroscience 20:3387-3400.

Qian N, Andersen RA (1994) Transparent motion perception as detection of unbalanced motion signals II: Physiology. Journal of Neuroscience 14:7367-7380.

Raz A, Buhle J (2006) Typologies of attentional networks. Nature Reviews Neuroscience 7:367-379. 
Recanzone GH, Wurtz RH (2000) Effects of attention on MT and MST neuronal activity during pursuit initiation. Journal of Neurophysiology 83:777-790.

Recanzone GH, Wurtz RH, Schwarz U (1997) Responses of MT and MST neurons to one and two moving objects in the receptive field. Journal of Neurophysiology 78:2904-2915.

Reynolds JH, Heeger DJ (2009) The normalization model of attention. Neuron 61:168-185.

Reynolds JH, Chelazzi L (2004) Attentional modulation of visual processing. Annual Review of Neuroscience 27:611-647.

Reynolds JH, Chelazzi L, Desimone R (1999) Competitive mechanisms subserve attention in macaque areas V2 and V4. Journal of Neuroscience 19:1736-1753.

Reynolds JH, Desimone R (2003) Interacting roles of attention and visual salience in V4. Neuron 37:853-863.

Roberts M, Delicato LS, Herrero J, Gieselmann MA, Thiele A (2007) Attention alters spatial integration in macaque $\mathrm{V} 1$ in an eccentricity-dependent manner. Nature Neuroscience 10:1483-1491.

Roelfsema PR, Tolboom M, Khayat PS (2007) Different Processing Phases for Features, Figures, and Selective Attention in the Primary Visual Cortex. Neuron 56:785-792.

Rust NC, Mante V, Simoncelli EP, Movshon JA (2006) How MT cells analyze the motion of visual patterns. Nature Neuroscience 9:1421-1431.

Saenz M, Buracas GT, Boynton GM (2002) Global effects of feature-based attention in human visual cortex. Nature Neuroscience 5:631-632.

Salinas E, Abbott LF (1997) Invariant visual responses from attentional gain fields. Journal of Neurophysiology 77:3267-3272.

Salzman CD, Britten KH, Newsome WT (1990) Cortical microstimulation influences perceptual judgements of motion direction. Nature 346:174-177.

Schmolesky M (2007) The primary visual cortex. In: Webvision (Kolb, H., Nelson R., Fernandez, E. and Jones, B. eds), http://webvision.med.utah.edu/

Sceniak MP, Ringach DL, Hawken MJ, Shapley R (1999) Contrast's effect on spatial summation by macaque V1 neurons. Nature Neuroscience 2:733-739.

Shapley R, Hawken M, Ringach DL (2003) Dynamics of orientation selectivity in the primary visual cortex and the importance of cortical inhibition Neuron 38:689699. 
Simoncelli EP, Heeger DJ (1998) A model of neural responses in visual area MT. Vision Research 38:743-761.

Sincich LC, Horton JC (2005) The circuitry of V1 and V2: integration of color, form, and motion. Annual Review of Neuroscience 28:303-326.

Slotnick SD, Schwarzbach J, Yantis S (2003) Attentional inhibition of visual processing in human striate and extrastriate cortex. Neurolmage 19:1602-1611.

Snowden RJ, Erickson RG, Treue S, Andersen RA (1990) Transparent motion stimuli reveal divisive inhibition in area MT of macaque. In: Association for the Research in Ophthalmology Ann. Meeting, vol. 31, p 399.

Snowden RJ, Treue S, Andersen RA (1992) The response of neurons in areas V1 and MT of the alert rhesus monkey to moving random dot patterns. Experimental Brain Research 88:389-400.

Snowden RJ, Treue S, Erickson RE, Andersen RA (1991) The response of area MT and V1 neurons to transparent motion. Journal of Neuroscience 11:2768-2785. Snowden RJ, Verstraten FAJ (1999) Motion transparency: Making models of motion perception transparent. Trends in Cognitive Sciences 3:369-377.

Spitzer H, Desimone R, Moran J (1988) Increased attention enhances both behavioral and neuronal performance. Science 240:338-340.

Spitzer H, Richmond BJ (1991) Task difficulty: ignoring, attending to, and discriminating a visual stimulus yield progressively more activity in inferior temporal neurons. Experimental Brain Research 83(2): 340-348.

Super H, Roelfsema PR (2005) Chronic multiunit recordings in behaving animals: advantages and limitations. Progress in Brain Research 147:263-282.

Suzuki N, Watanabe O (2009) Perceptual costs for motion transparency evaluated by two performance measures. Vision Research 49:2217-2224.

Thiele A, Pooresmaeili A, Delicato LS, Herrero JL, Roelfsema PR (2009) Additive Effects of Attention and Stimulus Contrast in Primary Visual Cortex. Cerebral Cortex 19:2970-2981.

Tootell RBH, Hadjikhani N, Hall EK, Marrett S, Vanduffel W, Vaughan JT, Dale AM (1998) The retinotopy of visual spatial attention. Neuron 21:1409-1422.

Treue S (2001) Neural correlates of attention in primate visual cortex. Trends in Neuroscience 24:295-300.

Treue S (2003) Visual attention: the where, what, how and why of saliency. Current Opinion in Neurobiology 13:428-432. 
Treue S, Hol K, Rauber HJ (2000) Seeing multiple directions of motion Physiology and psychophysics. Nature Neuroscience 3:270-276.

Treue S, Martinez-Trujillo JC (1999) Feature-based attention influences motion processing gain in macaque visual cortex. Nature 399:575-579.

Treue S, Maunsell JHR (1996) Attentional modulation of visual motion processing in cortical areas MT and MST. Nature 382:539-541.

Tsao DY, Freiwald WA, Tootell RB, Livingstone MS (2006) A cortical region consisting entirely of face-selective cells. Science 311:670-674.

Turatto M, Vescovi M, Valsecchi M (2007) Attention makes moving objects be perceived to move faster. Vision Research 47:166-178.

Ungerleider LG, Mishkin M (1982) Two cortical visual systems. In: Analysis of Visual Behavior(Ingle, D. J. et al., eds), pp 549-586 Cambridge: MIT Press.

Ungerleider LG, Desimone R. 1986. Cortical Connections of Visual Area Mt in the Macaque. Journal of Comparative Neurology 248: 190-222

Van Essen DC, Gallant JL (1994) Neural mechanisms of form and motion processing in the primate visual system. Neuron 13:1-10.

Van Essen DC, Maunsell JHR (1983) Hierarchical organization and functional streams in the visual cortex. Trends in Neuroscience 370-375.

Van Essen DC, Maunsell JHR, Bixby JL (1981) The middle temporal visual area in the macaque: myeloarchitecture, connections, functional properties and topographic organization. Journal of Comparative Neurology 199:293-326.

Wandell BA, Smirnakis SM(2009) Plasticity and stability of visual field maps in adult primary visual cortex, Nature Reviews Neuroscience, doi:10.1038/nrn2741

Wannig A, Rodriguez V, Freiwald WA (2007) Attention to surfaces modulates motion processing in extrastriate area MT. Neuron 54:639-651.

Watanabe $\mathbf{O}$, Kikuchi M (2005) Nonlinearity of the population activity to transparent motion. Neural Networks 18:15-22.

William J (1890) The principles of Psychology. New York, Henry Holt, Vol. 1, 403404

Williford T, Maunsell JHR (2006) Effects of spatial attention on contrast response functions in macaque area V4. Journal of Neurophysiology 96:40-54.

Womelsdorf T, Anton-Erxleben K, Pieper F, Treue S (2006) Dynamic shifts of visual receptive fields in cortical area MT by spatial attention. Nature Neuroscience 9:1156-1160. 
Womelsdorf T, Anton-Erxleben K, Treue S (2008) Receptive field shift and shrinkage in macaque middle temporal area through attentional gain modulation. Journal of Neuroscience 28:8934-8944.

Womelsdorf T, Fries P, Mitra PP, Desimone R (2006) Gamma-band synchronization in visual cortex predicts speed of change detection. Nature 439:733-736.

Yantis S, Serences JT (2003) Cortical mechanisms of space-based and objectbased attentional control. Current Opinion in Neurobiology 13.

Yoshor D, Ghose GM, Bosking WH, Sun P, Maunsell JH (2007) Spatial attention does not strongly modulate neuronal responses in early human visual cortex. Journal of Neuroscience 27:13205-13209.

Zemel RS, Pillow J (2000) Encoding multiple orientations in recurrent networks. Neurocomputing 32:609-616.

Zihl J, Von Cramon D, Mai N. (1983) Selective Disturbance of Movement Vision after Bilateral Brain Damage. Brain 106: 313-40 


\title{
Curriculum Vitae
}

\author{
VALESKA MARIJA StEPHAN
}

Personal details

\begin{tabular}{ll}
\hline Date of birth & $2^{\text {nd }}$, September 1979 \\
Place of birth & Siegen, Germany
\end{tabular}

\section{Scientific education}

since 2008

$1999-2007$

1999

Teaching experience

2008-2012

2008-2010

2008-2012

2008-2012
Graduate student - Center for Systems Neuroscience, Graduate School for Neuroscience and Molecular Biosciences, Göttingen Germany, Graduate student, working at the German Primate Center, Cognitive Neuroscience Laboratory, Göttingen, Germany

Advisor: Prof. Dr. Stefan Treue

Undergraduate studies in biology, Kassel University Kassel, Germany

Diploma thesis: 'The influence of attention on the receptive field in the rhesus monkey', German Primate Center, Cognitive Neuroscience Laboratory, Göttingen, Germany

Abitur, Phoenix-Gymnasium, Dortmund, Germany
Regular supervision of several methodcourses and practicals, German Primate Center, Göttingen

Several tutorials on visual perception and attention for undergraduate students in the Graduate Program Neurosciences, Göttingen

Regular supervision of several neurobiology seminars, University of Göttingen

Supervision of several practical students, German Primate Center, Göttingen 


\section{Courses, internships \& workshops}

April 2012

March 2010

October 2008

September 2008

August - September 2005

May - August 2005
"Kommunikationstraining für Wissenschaftler" by the Klaus Tschira Stiftung, German Primate Center, Göttingen (Germany)

Plexon Technical Workshop, Plexon Inc, Dallas (USA)

NWG-Workshop „Analysis and Models in Neurophysiology", Bernsteincenter for Computational Neuroscience, Freiburg (Germany)

NWG-Workshop „Psychophysical methods and Matlab programming", University of Gießen (Germany)

Internship at „Nature“ Journal, Office Munich (Germany)

Internship at "Genius - Science \& Communication“, Darmstadt (Germany)

\section{Publications}

Anton-Erxleben K, Stephan VM, Treue S Attention reshapes center-surround receptive-field structure in macaque cortical area MT. Cerebral Cortex 19(10): 2466-2478

Lochte A, Stephan VM, Kozyrev K, Treue S Attentional modulation of the tuning of neurons in macaque area MT to the direction of transparent motion patterns. Prepared for submission

Stephan VM, Liu R-F, Treue S Spatial attentional modulation of multi-unit activity in macaque V1. Prepared for submission

\section{Abstracts \& Conference contributions}

Stephan VM, Treue S (2012) Spatial attentional modulation of multi-unit activity in macaque V1. Society for Neuroscience Abstracts.

Stephan VM, Treue S (2012) Spatial attentional in primary visual cortex. Primate Neurobiology Meeting Tübingen. 
Lochte A, Stephan VM, Kozyrev V, Treue S (2010) Attentional modulation of the tuning of neurons in macaque area MT to the direction of transparent motion patterns. Primate Neurobiology Meeting Tübingen

Lochte A, Stephan VM, Kozyrev V, Witt A, Treue S (2009) Attentional modulation of the tuning of neurons in area MT to the direction of transparent motion. Society for Neuroscience Abstracts.

Lochte A, Stephan VM, Kozyrev V, Battaglia D, Treue S (2009) Attentional modulation of the tuning of neurons in macaque area MT to the direction of transparent motion patterns. Frontiers Conferences

Lochte A, Stephan VM, Kozyrev V, Treue S (2009) The influence of attention on the processing of transparent motion. Göttingen Neurobiology Meeting

Lochte A, Stephan VM, Kozyrev V, Treue S (2009) Attentional alteration of direction tuning of neurons in macaque area MT to transparent motion. Primate Neurobiology Meeting Göttingen.

Lochte A, Stephan VM, Kozyrev V, Witt A, Treue S (2009) Attentional modulation of the tuning of neurons in area MT to the direction of transparent motion. Frontiers in Computational Neuroscience. Bernstein Conference on Computational Neuroscience. 